\title{
An end-to-end solution for automation of biological protocols
}

Ph.D. Thesis

Author: Vishal Gupta, MSc.

Supervisor: Dr. Alfonso Vicente Rodríguez-Patón Aradas 2017 
Dedicated to my family and friends 


\section{Acknowledgements}

First and foremost, I would like to thank my advisor Alfonso Rodríguez-Patón. His unwavering support, patience and freedom to explore new avenues has been driving force behind this work. I have learnt immensely under his tutelage, especially to think independently and find simple yet powerful solutions which should be useful to the community. Thank you.

I would like to thank my colleagues who worked tirelessly with me on the EVOPROG project. Ismael, Jesús, Ángel and Iván. Ángel and Jesús, your patience and hard work has made this work possible.

I would also like to thank the EVOPROG project partners Dr. Alfonso Jaramillo, Dr. Victor de Lorenzo, Dr. Leroy Cronin and Dr. Mark Isalan for their encouragement during the project.

I would like to thank my colleagues in the Laboratory of Artificial Intelligence: Willy, Martin, Paula, Marcos, Ismael, Gustavo, Sandra and Luís. It was a privilege to be on this unforgettable roller coaster ride together.

Last but not the least, I would like to thank my parents, my brother, my extended family and friends who have been through thick and thin with me. I could have not done it without you. 


\section{CONTENTS}

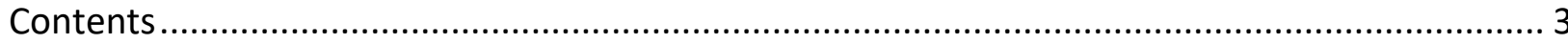

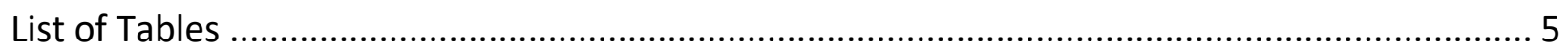

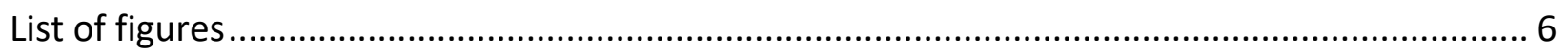

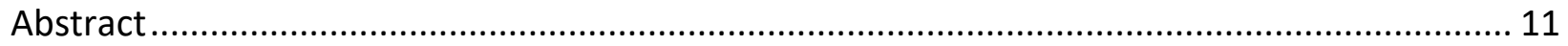

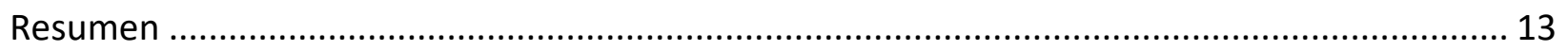

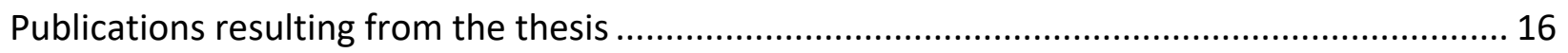

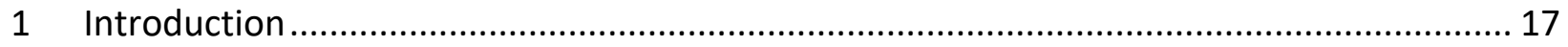

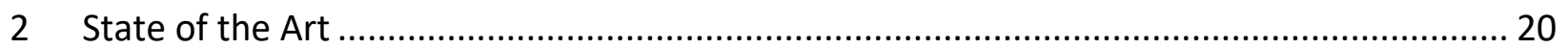

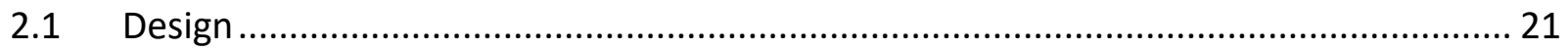

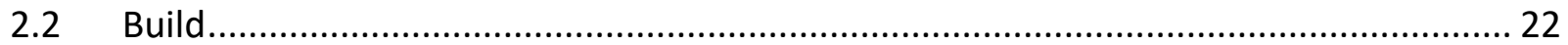

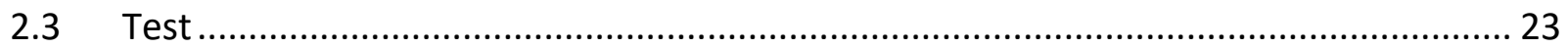

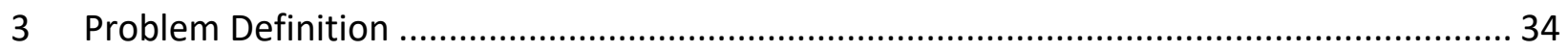

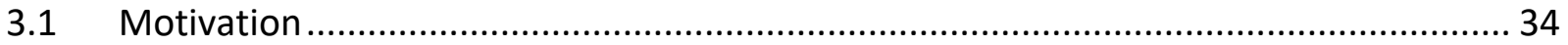

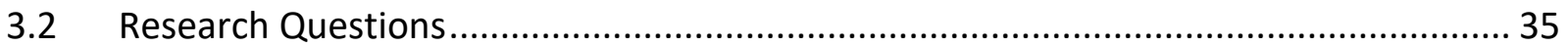

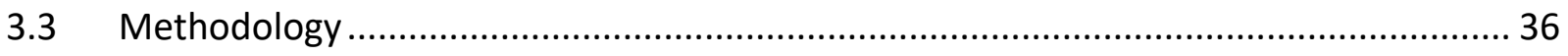

4 Proposed Solution: BioBlocks Automation Framework .............................................. 38

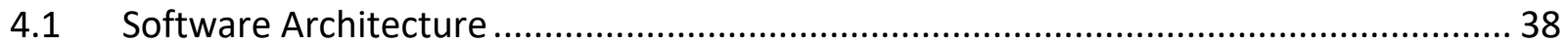

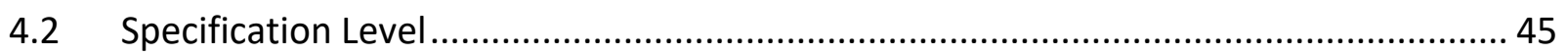

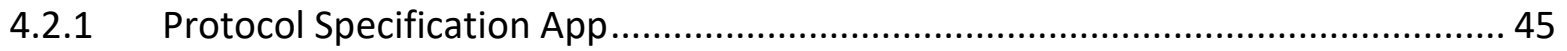

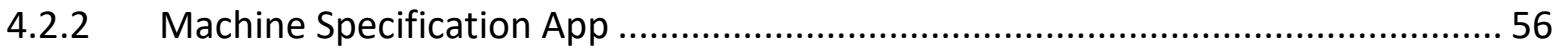

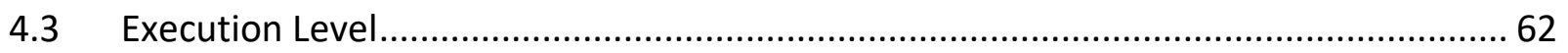




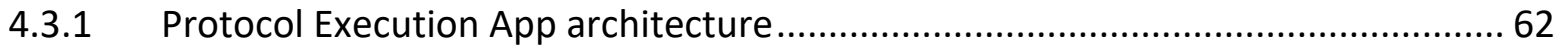

4.3.2 Protocol Execution App functionalities ........................................................... 64

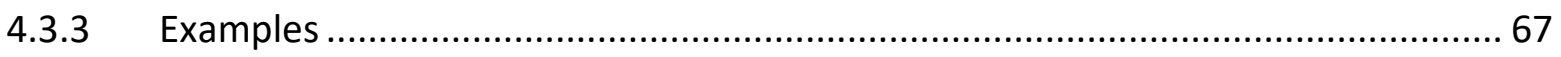

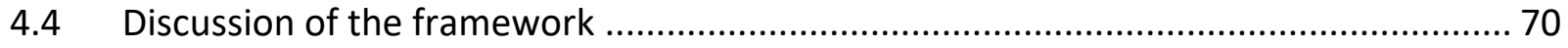

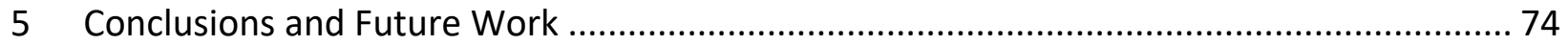

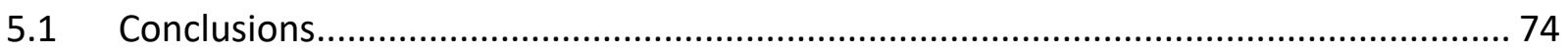

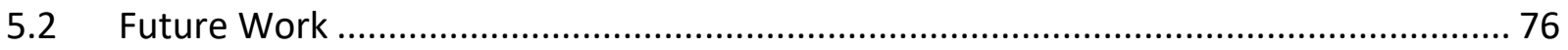

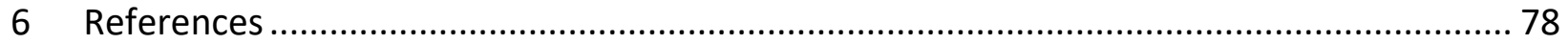

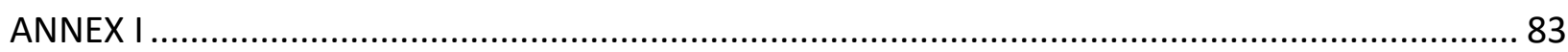

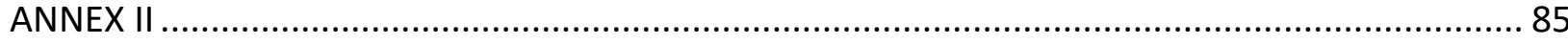

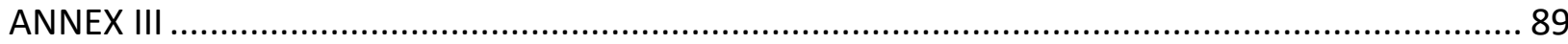

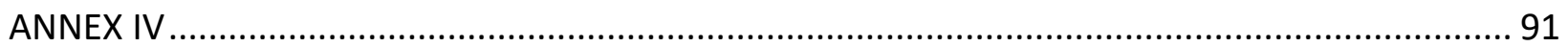




\section{LIST OF TABLES}

Table 1 Graphical Notation used in the Protocol Workflow

52

Table 2 List of icons used to represent the machine layout in the Machine Specification App.

The different icons represent different parts of a container

60

Table 3 List of BioBlocks and their respective Autoprotocol instructions

83 


\section{LIST OF FIGURES}

Figure 1 Working with a Pipette - Two pipettes with different pipetting capacities (A). A pipette's body is held in the palm and the thumb is put on the piston (B). The aspiration or dispensing of liquids is done by pushing the piston up or down with the thumb (C).

Figure 2 Classification of academic and commercial contributions in the field of automation: An attempt has been made to show the contributions according to their functions by placing them in the BUILD, TEST and/or DESIGN part. They yellow dotted circles in the Design section further classify the tools based on their function i.e. if they are DNA level (top yellow) specification tools, Function level (middle yellow) or System Level (bottom yellow). 23

Figure 3 Autoprotocol Instructions and Syntax - Autoprotocol is a formal language to specify experiments in biology. The type of experiments that can be specified are mentioned under instructions (left). An example of a thermocycling experiment using the Autoprotocol syntax (Right) ${ }^{46}$. $\quad 25$

Figure 4 Abstraction layers in BioStream - BioStream required both biologists for protocol description and hardware developers for protocol execution. The BioStream library translates the protocol in BioStream to the Fluidic Instruction Set Architecture ${ }^{54}$. 28

Figure 5 Different outputs of Biocoder - A protocol of plasmid Extraction in Biocoder written in C (right) is translated to English Output (top left) and a task graph (bottom left) ${ }^{55}$. 29

Figure 6 The software tool flow of the Microfluidic Hardware Description Language IDE - The software uses the protocol specification from Biocoder and produces a layout of the microfluidic device and the instructions that need to execute the protocol over it. It also produces a simulation result of the protocol ${ }^{62} . \quad 32$

Figure 7 Logical View of the Software Architecture- The BioBlocks Framework consists of two levels; specification level for protocol and machine specification and execution level for execution of the protocol. 39 
Figure 8 Developers View of the Software Architecture- The different modules developed are depicted. The Blue boxes belong to the Specification Level and the Maroon boxes belong to the Execution level. The Grey boxes are part of the Hardware. The different modules are the Protocol Specification GUI (1), Machine Specification GUI (2), Protocol translator (3), Machine translator (4), Protocol-Machine compatibility (5), Execution Manager (6), Plug-in Manager (7), Communications Manager (8), Serial Communication (9) and the Simulator (10). 40

Figure 9 Screenshot of the BioBlocks Automation Framework Main Page. A brief description of the framework along with the options to 'Specify a Protocol', Specify a Machine' and 'Automate a Protocol' are present. Users can click on the tabs to access the required option. 42

Figure 10 Screenshot of the Protocol Specification App Option in the BioBlocks Automation Framework. A brief description of the App along with a link to the App is provided. The App opens in a new browser Tab. 43

Figure 11 Screenshot of the Machine Specification App page in the BioBlocks Automation Framework. A brief description of the App along with a link to the App is provided. The App opens in a new browser Tab. 43

Figure 12 Screenshot of the Protocol Execution App page in the BioBlocks Automation Framework. A brief description of App along with a link to the App is provided. The App opens in a new browser Tab.44

Figure 13 BioBlocks Library - A snapshot of some of the Blocks available in the BioBlocks Library. The container blocks are shown in blue, the operation blocks are shown in green and the organization blocks are shown in black. Blockly's native 'conditional blocks' (orange) have been modified to allow for specification of protocols using conditions. 46

Figure 14 Protocol Specified in BioBlocks - The operation block (green), the container block (blue), the organization block (black) and the native Blockly input block (purple) on the left can be attached to each other to form a protocol (right). In the above example a PCR experiment is designed. The shapes of the block guide the user to specify a protocol in an easy manner. 47 
Figure 15 Specifying conditional protocols using BioBlocks - In the above example a conditional protocol based on an OD measurement has been specified (right). Depending on the OD value that IF OD $<1$, a transfer is made from Tube $A$ to Tube $B$ or from Tube $A$ to Tube $C$. The protocol workflow is equipped to represent the conditional operation which is highlighted in a grey box (right). 48

Figure 16 Advanced conditional operation specification - BioBlocks uses a very powerful native Blockly feature to allow specification of advanced conditional operations. Users can click on the settings icon and drag and drop 'else' or 'else if' blocks (blue) to add more conditions to the IF block. 49

Figure 17 Multiple outputs of protocol specified in BioBlocks - The PCR example in Figure 14 generates the three outputs automatically in real-time. An English transition of the specified protocol, Output Code which is machine readable and a Protocol workflow for visual aid are generated. $\quad 50$

Figure 18 Visual Manipulation using connector types - There are two types of jig-saw like connectors available in Blockly. Connector 1 has been used to attach operation blocks, organization blocks and conditional blocks. Connector 2 is used for attaching container blocks and inputs to operation blocks. 54

Figure 19 Visual Manipulation using Expanding/Collapsing Blocks - BioBlocks use a very powerful Blockly feature of Collapsing and Expanding Blocks to allow for improved visual manipulation of specified protocols. The protocol on the left can be collapsed for easier visual manipulation. The collapsed blocks on the right can be expanded back (restored) to its previous state. 55

Figure 20 Dynamic Menu for progressive disclosure of inputs - The container block on the left (blue) displays more options depending on the operation block it has been attached to. In the above example, the container blocks on the right (blue) displays the number of steps it needs to thermocycle when used with a Thermocycling Operation Block. 55 
Figure 21 Creating a machine from the plugin library and parts - New parts (blue) can be created in the Machine Specification App (left). Pre-loaded plugins (orange) can be selected from the library (bottom right). The parts and plugins can be attached together along with machine layout block (black, top right). 59

Figure 22 Machine Specification App example - A continuous flow millifluidic device called a Turbidostat was specified in the App. The block-based specification using parts (blue) and plugins (orange) is shown on the left. The automated real-time output of the machine layout is displayed on the right. 61

Figure 23 Architecture of the Protocol Execution App - The different modules present in the Protocol Execution App are shown in Maroon. The protocol and the machine specifications are first transformed into internal graphs (1,2), followed by a compatibility check (3) and finally instructions are generated after gathering device specific information from the plugin Manager $(4,5)$ which are sent to the micro-controller $(6,8)$. The user also has an option to generate a simulation of the protocol execution (7). 63

Figure 24 Graph Model of the protocol - The protocol is transformed to a graph model for internal representation so that operations can be performed over it. The nodes are operations and the edges are conditions. 65

Figure 25 Turbidostat Protocol in BioBlocks in the BioBlocks framework -the Continuous flow operation block (green) a Turbidostat can be easily specified. In the protocol, the Rate has been set proportional to the change in OD value. 68

Figure 26 The Execution of the Turbidostat using the Protocol Execution App - The Turbidostat Protocol (Figure 25) and the Turbidostat setup (Figure 22) were uploaded in the Protocol Execution App. The resultant generated instructions generated as displayed in the console is shown above. The App prompts user to click OK after loading the respective containers. The output has been condensed for brevity. 69

Figure 27 Aimed Interoperability of BioBlocks - BioBlocks was designed to be able to connect with multiple solutions like Automated Equipment Pipelines likes Transcriptics, Pipetting Robots 
like OpenTrons, flow control devices like Aqua and EvoCoder and Bio-design and workflow languages like Antha. 72

Figure 28 Schematic of the EvoCoder architecture: The three different operation modes Scheduling, Protocol Execution and Test design are depicted in the schematic. The software modules in the compiler are shown in green boxes. The input and output files are shown in white boxes. The software modules on the Arduino side are shown in pink. Unlike the BioBlocks Automation Framework, EvoCoder required a text input of the machine layout. 85 


\section{ABSTRACT}

The inability to reproduce the results of biological research has long been the elephant in the room. Non-reproducibility of results causes billions of dollars in losses in money, time and other resources. This slows down over all scientific progress. There are some important factors which contribute to the reproducibility problem. There is ambiguity in experimental method specification, human error introduced while conducting experiment and lack of data sharing standards. Recently, some interesting approaches have been developed to alleviate the reproducibility problem. They are the use of 1) programming languages to removing ambiguity in experimental method specification, 2) use of robotic automation for conducting experiment to reduce human error and 3) use standards and ontologies for improving data sharing. The first two solutions have still met with limited success. This is because use of programming languages requires the user to have prior programming knowledge. Even if the user has coding skills, different automation solutions use different programming languages. This requires a lot of time and effort on the researcher's part as they need to learn different languages to use different automation solutions. Most automated solutions today come with a graphical user interface (GUI) which circumvents the programming obstacle. However, different automated solutions again have different GUIs which need considerable effort to master.

Programing languages and Automation were used as an approach to tackle the reproducibility problem. Automation also offers the additional benefits of improving efficiency and productivity in the lab. A web-based end to end automation framework was developed called the BioBlocks Automation Framework (BAF). BAF allows users to specify their experiments (Protocol Specification App) along with the machine (wet-lab hardware) on which it would it automated (Machine Specification App), followed by generation of instructions for actual execution (Protocol Execution App). It allows users to automate their experiments without the need for any prior programming knowledge. It uses a block-based graphical interface based on MIT's Scratch and Google's Blockly. They use a jig-saw like interface to teach beginners how to program. It makes it easier to learn programming as users do not need to learn syntaxes. Using 
the BAF, users can specify their protocols and machines by a simple drag and drop mechanism. The working of the three main components of BAF is explained as follows:

- A protocol specified in the Protocol Specification App using blocks is automatically translated in real-time to give multiple outputs. It is translated to English for cross-validation, a Protocol workflow for visual aid and an Output code in which the protocol is represented in JSON format for later automation. The protocol specified are platform agnostic i.e. they are independent of the platform on which they will be executed. The specified protocols are also unambiguous and can be executed manually using English Translation.

- A machine (wet-lab hardware) specified in the Machine Specification App using blocks is translated automatically in real-time to generate a machine layout to serve as visual aid and Output code (representation in JSON format). It currently has support for millifluidic devices. However, the modular architecture of the framework will allow users to specify other microfluidic devices as well. It follows a unique part-machine specification paradigm to make the specification extensible to other hardware platforms.

- Using the Protocol Execution App, the specified protocol can be automated over the specified machine. The App automatically checks if the protocol and machine are compatible, followed by routing the flow over the machine setup and finally generating machine instructions to control the actuation.

BioBlocks framework is a general-purpose end to end automation framework. It is not tied to a single hardware platform and the protocols specified are independent of the platform. The protocols and machines described in the framework can be easily saved, shared and modified. It has been designed to allow it to connect with other protocol specification tools and other hardware platforms. This work aims to lower the entry barrier for non-computer scientist to automation tools by removing the programming bottleneck. It aims to enable biologists, hardware engineers and Do-it-Yourself enthusiasts by providing them a platform where experiments can be designed, executed and shared. 


\section{RESUMEN}

La incapacidad de reproducir los resultados de la investigación biológica ha sido durante mucho tiempo el problema al que todos han hecho la vista gorda. La incapacidad de reproducir los resultados malgasta el tiempo y otros recursos causando pérdidas de miles de millones de dólares. Esto retrasa todo el progreso científico. Algunos factores importantes que contribuyen al problema de "la irreproducibilidad" son: la especificación del método experimental es ambigua, se producen errores humanos durante la realización de experimentos y la falta de normas de intercambio de datos. Recientemente, se han desarrollado algunos enfoques interesantes para aliviar estos problemas. Los más importantes son: 1) El uso de lenguajes de programación para eliminar la ambigüedad en la especificación del método experimental, 2) el uso de la automatización robótica para llevar a cabo experimentos y así reducir el error humano y 3) utilizar estándares y ontologías para mejorar el intercambio de datos. Las dos primeras soluciones, por el momento, han tenido un éxito limitado. Esto se debe a que para usar lenguajes de programación se requiere que el usuario tenga conocimientos técnicos previos. Incluso si el usuario tiene estos conocimientos, diferentes soluciones de automatización utilizan diferentes lenguajes de programación. Esto requiere mucho tiempo y esfuerzo por parte de los investigadores, ya que necesitan aprender diferentes idiomas para usar las diferentes soluciones de automatización. La mayoría de las soluciones automatizadas de hoy en día vienen con una interfaz gráfica de usuario (GUI) que evita el obstáculo de programación. Sin embargo, cada solución tiene diferentes GUI que requieren un esfuerzo considerable para dominar. La automatización también ofrece otros beneficios adicionales como mejorar la eficiencia y la productividad en el laboratorio. Se ha desarrollado un Framework de automatización de extremo a extremo basado en tecnología Web y denominado BioBlocks Automation Framework (BAF). BAF permite que los usuarios especifiquen sus experimentos (aplicación de especificación de protocolos) junto con la máquina en la que se automatizaría (aplicación de especificación de máquinas). Además, permite generar instrucciones para la ejecución de un experimento en tiempo real. Los usuarios pueden automatizar sus experimentos sin la necesidad de ningún conocimiento previo de programación porque utiliza una interfaz gráfica 
basada en bloques y en MIT's Scratch y Blockly de Google. Estos últimos utilizan un paradigma visual basado en puzzles que se usa para enseñar a los principiantes a programar, este paradigma visual tiene una curva de aprendiz más alta que los paradigmas clásicos ya que los usuarios no necesitan aprender sintaxis. Utilizando BAF, los usuarios pueden especificar sus protocolos y máquinas mediante un sencillo mecanismo "drag-and-drop". El funcionamiento de los tres componentes principales de BAF se explica a continuación:

- Un protocolo especificado, usando la aplicación de especificación de protocolos y los bloques disponibles, se traduce en tiempo real a diferentes formatos: Por un lado, se traduce al inglés para la validación cruzada. Además, se genera un flujo de trabajo que actuá como ayuda visual a la hora de validarlo. Por último, se traduce a un "código maquina", en formato JSON, que será utilizado posteriormente para automatizar dicho protocolo. El protocolo especificado es independiente de la plataforma en la que se ejecutará. Los protocolos especificados también son inequívocos y se pueden ejecutar manualmente utilizando la traducción en inglés.

- Una máquina especificada, usando la aplicación de especificación de máquina y los bloques disponibles, se traduce en tiempo real para generar una imagen del diseño de la máquina que sirva como ayuda visual y un "código de salida", en formato JSON, que será usado posteriormente para la automatización de protocolos en dicha máquina. Actualmente cuenta con soporte para dispositivos milifluídicos. Sin embargo, la arquitectura modular del "framework" permitirá en el futuro especificar otros dispositivos como los microfluídicos o los robots de pipetas. La especificación sigue un paradigma máquina-componente que lo hace fácilmente ampliable a estos nuevos tipos de dispositivos.

- Mediante la aplicación de "Ejecución de protocolos", un protocolo especificado se puede automatizar sobre una máquina, previamente especificada. La aplicación comprueba automáticamente si un protocolo y una máquina son compatibles, usa un algoritmo de enrutamiento para acomodar los flujos que indique el protocolo sobre la configuración de la máquina y finalmente genera instrucciones para que la máquina lleve a cabo el protocolo. 
BAF es un "framework" de automatización de extremo a extremo de uso general. No está vinculado a una sola plataforma de automatización y los protocolos especificados son independientes de dicha plataforma. Los protocolos y máquinas descritos en él pueden ser guardados, compartidos y modificados fácilmente. Se ha diseñado para permitir que se conecte con otras herramientas de especificación de protocolo y otras plataformas de automatización. Este trabajo tiene como objetivo reducir la barrera de entrada para los científicos no informáticos a las herramientas de automatización eliminando el cuello de botella que crea la programación. Su objetivo es proporcionar a: los biólogos, los ingenieros de hardware y los entusiastas del "Do-It-Yourself" una plataforma donde los experimentos puedan ser diseñados, ejecutados y compartidos. 


\section{PUBLICATIONS RESULTING FROM THE THESIS}

$\underline{\text { Articles }}$

Gupta, V., Irimia, J., Pau, I. \& Rodriguez-Paton, A. BioBlocks : Programming protocols in biology made easier. ACS Synthetic Biology (2017). doi:10.1021/acssynbio.6b00304

BioBlocks: Programming protocols in biology made easier. Vishal Gupta, Jesús Irimia, Iván Pau, Alfonso Rodríguez-Patón. bioRxiv 081075; doi: https://doi.org/10.1101/081075.

$\underline{\text { Talks }}$

Title - BioBlocks: A web-based visual environment for programming experimental protocols in biological sciences.

Authors - Vishal Gupta, Jesús Irimia, Iván Pau, Alfonso Rodríguez-Patón.

Workshop/Conference - International Workshop on Bio-Design Automation (IWBDA), 16.08.2016 - 18.08.2016, Newcastle Upon Tyne, United Kingdom.

\section{$\underline{\text { Poster }}$}

Title - Drawing Biological Protocols: A computer aided approach for biological protocol description and execution.

Authors- Vishal Gupta, Ismael Gómez, Alfonso Rodríguez-Patón.

Workshop/Conference - International Synthetic and Systems Biology Summer School (SSBSS), 05.07.2015 - 09.07.2015, Taormina, Italy.

\section{Business Competition}

Title-BioBlocks

Team - Vishal Gupta, Angel Panizo Lledot, Alfonso Rodríguez-Patón.

Award - Top prize of $1000 €$ in the first round of UPMs prestigious annual business competition called ActuaUPM. 


\section{INTRODUCTION}

Non-reproducibility of scientific results is a critical problem affecting all disciplines of science. It causes billions of dollars of losses in money, time and other resources. Consequently, it slows down over all scientific progress. Multiple recent studies have shown that not all scientific results are reproducible ${ }^{1}$. Non-reproducibility is at the level of conducting the experiment and at the level of data analysis. There are many studies and initiatives in place today to ensure reproducibility at the level of data analysis ${ }^{2-6}$. This is being done by making the data sets available for scientists to test. However, there is very little focus on the method which is used to generate the data in the first place. This work will focus mainly on the method, specifically wet-lab molecular and microbiology methods to improve the reproducibility of scientific research. There are many factors responsible for the reproducibility crisis. The most important factors are:

Ambiguity in method specification - Unfortunately, there is a lot of ambiguity present in the textual description of many published experimental method (protocols). Ambiguity can be due to use of unquantifiable and missing parameters, undefined terms etc. An experimental method can contain unquantifiable terms like 'overnight incubation' and 'volume was made up to'. The first term belongs to the category where the 'time is unknown' and the second term belongs to the category where the 'quantity is unknown'. There are many other terms which fall into this category and are frequently used while describing the methods. These terms introduce ambiguity as overnight incubation could mean any value between 7-15 hours. The 2fold time difference can introduce a lot of variability in an experimental outcome which leads to irreproducibility of results. Another reason for ambiguity is that most journals have a page/word limits for different formats of publications (Article, Letter, Communications etc.). The method of conducting the experiment is usually reduced to a small section to stay within the word limit. This leads to ambiguity and non-reproducibility as researchers omit a lot critical information necessary for reproducing an experiment. 
Human Error - Experiments are conventionally conducted manually in most of the laboratories globally. Wet-lab experimentation in biology specifically benchwork, essentially consists of series of steps which involves manipulating small amounts of fluids. These fluids can be reagents like buffers, dyes or bio-entities like primers, plasmids, organisms etc. and are manipulated mostly using handheld pipettes. The use of electronic pipettes is usually limited to toxic liquids or for large volumes of liquid. An example is changing the media buffer while passaging cells. Pipetting requires a user to press down (apply pressure) on the pipette head, to aspirate and dispense volumes between different containers (tubes, multiwell plates etc.). There is a usually variation introduced between fluids transferred by different users and between fluids transferred by the same user. Pipettes are laboratory work horses and need to be maintained well to ensure reproducibility in pipetting volumes. Human error can also be due to the inability of researcher to conduct all the required steps, or to unintentionally skip certain steps in an experimental protocol (See Figure 1).
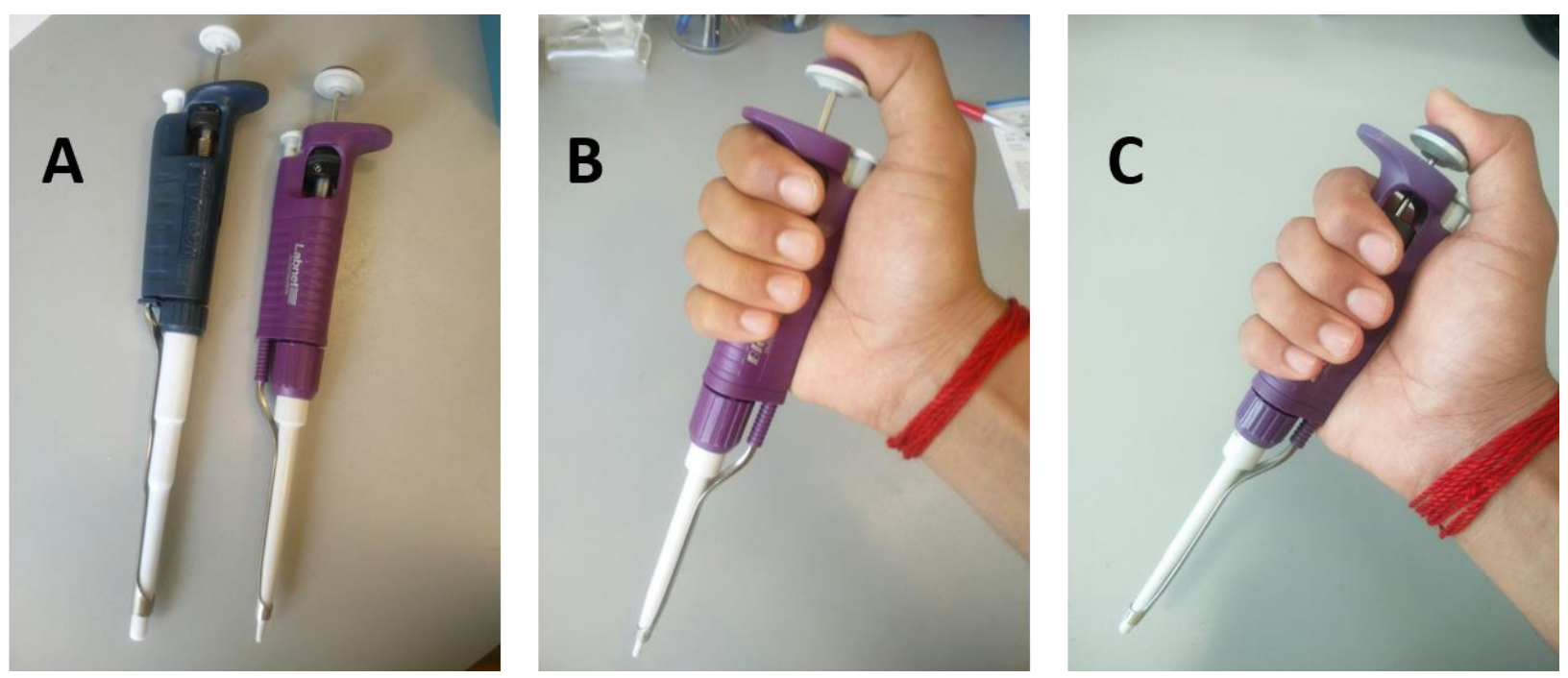

Figure 1 Working with a Pipette - Two pipettes with different pipetting capacities (A). A pipette's body is held in the palm and the thumb is put on the piston (B). The aspiration or dispensing of liquids is done by pushing the piston up or down with the thumb (C).

Lack of standards - There is always been a lag in creation of standards in biological sciences. This is due to the lack of our understanding of the complexity in biological systems and the 
required metrology to quantify their complexity. There have been initiatives in the last few decades (with the rise of genomics) to adapt standards for data sharing of natural sequences like FASTA, GenBank ${ }^{7,8}$. However, with the advent of next generation sequencing and synthesis, there is an been a massive increase of genomic data. Along with advent of Synthetic biology, sequence information needs to be stored and shared in specific contexts. A good example is storing them as parts or circuits that perform specific functions. There are initiatives which allow sharing of such context specific parts ${ }^{9-11}$. However, the curation of parts with reproducible behavior is a major problem. The parts can be uploaded by any user but are not validated by peers. Also, due to the lack of quantitative metrics and context specific dependence of activity it is very difficult to make the functioning of such entities reproducible ${ }^{12}$

Ontologies have been used to improve reproducibility as well. They are well-defined terminologies and their relationships in a domain that are machine readable. Biological Sciences has adapted Ontologies to map many of its domains like Genetics using Gene Ontology, EXACT for low-level description of experimental protocols, SMART etc. ${ }^{13-15}$

There are also other factors like lack of access to published data because of journal paywalls which influence the reproducibility of scientific results but are not discussed in this thesis, as they are beyond its scope. This thesis focusses on solving the first two problems mentioned above i.e. Ambiguity in method specification and Human Error. It refrains from attempting to solve the third problem that is to create a new standard.

The thesis is structured as follows - Chapter 2 deals with the 'State of the Art' in the field of Automation in biological experimentation from a Synthetic Biology perspective. The focus is on high-level languages for programming robotic platforms and other mili and microfluidic devices. Chapter 3 deals with the 'Problem Definition'. It mentions the shortcomings of the current approaches of solving the reproducibility crisis along with the Research Questions and Methodology. Chapter 4 elaborates the 'Proposed solution' to tackle the problems mentioned in the previous chapter. It discusses the end to end automated solution developed as a part of this research work. Chapter 5 discusses the 'Conclusions' and Future directions of this work. 


\section{STATE OF THE ART}

Biological Sciences has been evolving and moving from being less qualitative to more quantitative. There is a growing impetus to apply engineering principles to biology to allow for quantitative analysis. This in turn will allow for more systematic and rational engineering of complex biological organisms. These endeavors have given rise to the fields of Systems and Synthetic biology. Many different engineering metaphors are applied to find a suitable paradigm for biology. Parallels have been drawn between electronics engineering and biology to allow for classification of parts, devices and modules and systems. Computer Engineering metaphors have also been successfully applied where genes are akin to programs which when put inside a cell (computer) produces the outcome which is a phenotype. However, irrespective of the engineering method applied to biology reproducibility has been a major bottle neck ${ }^{12}$.

From the biological perspective, non-reproducibility is because genetic parts, devices and modules behave differently in different contexts. There is a big gap between their physical composition and their functional output; it is very context dependent. This is because of our limited understanding of the biological systems. There have been many attempts to improve the behavior of bio-entities (parts, devices) in different contexts by developing orthogonal parts, portable parts etc. reproducibility is not just because of the unpredictability of biological systems in different contexts. It is also because of a lack of standards in measuring biological activity accurately, lack of terminology/languages to specify different behaviors accurately without any ambiguity so that they can be used by the community.

One of the outcomes of adapting engineering principle in Synthetic Biology is the division of its workflow into the Design, Build and Test cycle. Some workflows also include a Learn or Simulate module in their cycle. This division abstracts the design of biological and experimental design from their building and testing. There are many software tools available, both academic and commercial for assistance in the design, build and test modules of the cycle. There are tools 
with specific functionality which help only in one of the other module or there are tools which help users optimize and automate the entire workflow. A review of the tools is given below -

\subsection{DESIGN}

To design new bio-circuits which can perform unique functions, specific sequences need to be manipulated in a precise manner. The CAD (Computer aided design) tools help designers the ease the complexity of the creation of innovative and effective bio-circuits. These CAD tools can be classified into different types based on the type of function they perform. GenoCAD ${ }^{16}$ is an early implementation for CAD tools in biology and is built upon formal grammar. Eugene ${ }^{17}$ is a human-readable and machine-executable language developed for the design process of synthetic biology at various levels of abstraction, such as sequences, parts, devices, and systems. It includes specification of (synthetic) biological components, support of data exchange standards, library management of design and components, specification of design constraints ("rules") on composing components into novel systems, built-in functions to enumerate rule-compliant designs automatically, control structures and functions for the specification of design synthesis. Synthetic Biology Open Language (SBOL) an open standard, is used for representing genetic designs through a standardized vocabulary of schematic glyphs (SBOL Visual) as well as a standardized digital format (the SBOL data model). SBOL has been very successful in gaining adoption from different research groups and journals as well ${ }^{9,10}$. Proto is a high-level language where the programmer describes the computation and the genetic regulatory network is automatically generated ${ }^{18}$. Gene Designer2.0 ${ }^{19}$ and Benchling ${ }^{20}$, both commercial ventures are DNA design tools with set of useful features like in silico cloning, codon optimization, back translation, CRISPR guide design, automated Gibson and Golden Gate cloning. Genome compiler ${ }^{21}$ is a similar tool with has direct connections to an array of DNA synthesis providers and lab facility services. Clotho is a framework for storage and exchange of biological data using the Clotho data model. It has built-in and user-developed apps for synthetic biological engineering. Pigeon (example app in Clotho) is a web-based software tool that renders textual descriptions of genetic circuit designs into graphical images using standardized visual elements. Merlin for Multiplex Automated Genome Engineering (MAGE) is a 
MAGE optimization tool. MAGE uses evolution for genome design and automation to shorten time scales. Billions of different mutant genomes can be generated per day. Given a list of desired oligos, Merlin aims to generate oligos for MAGE experiments to optimize genome-tooligo homology and oligo-to-oligo homology ${ }^{22-25}$. GEC by Microsoft is a programming language, which allows logical interactions between potentially undetermined proteins and genes to be

expressed in a modular manner ${ }^{16}$. Programs can be translated by a compiler into sequences of biological parts, a process which relies on logic programming and prototype databases containing known biological parts and protein interactions. Programs can also be translated to reactions, allowing simulations to be carried out. The language is a first step towards the automatic translation of high-level behavioral designs to low-level DNA code.

\subsection{BUILD}

Once, the required circuit is designed, it needs to be synthesized in part or full. There are many companies which have been working in this part for decades. However, some companies like DNA 2.0 and Integrated DNA technologies (IDT) are using innovative technologies to bring down the cost of synthesizing bio-parts ${ }^{26,27}$. 
BUILD

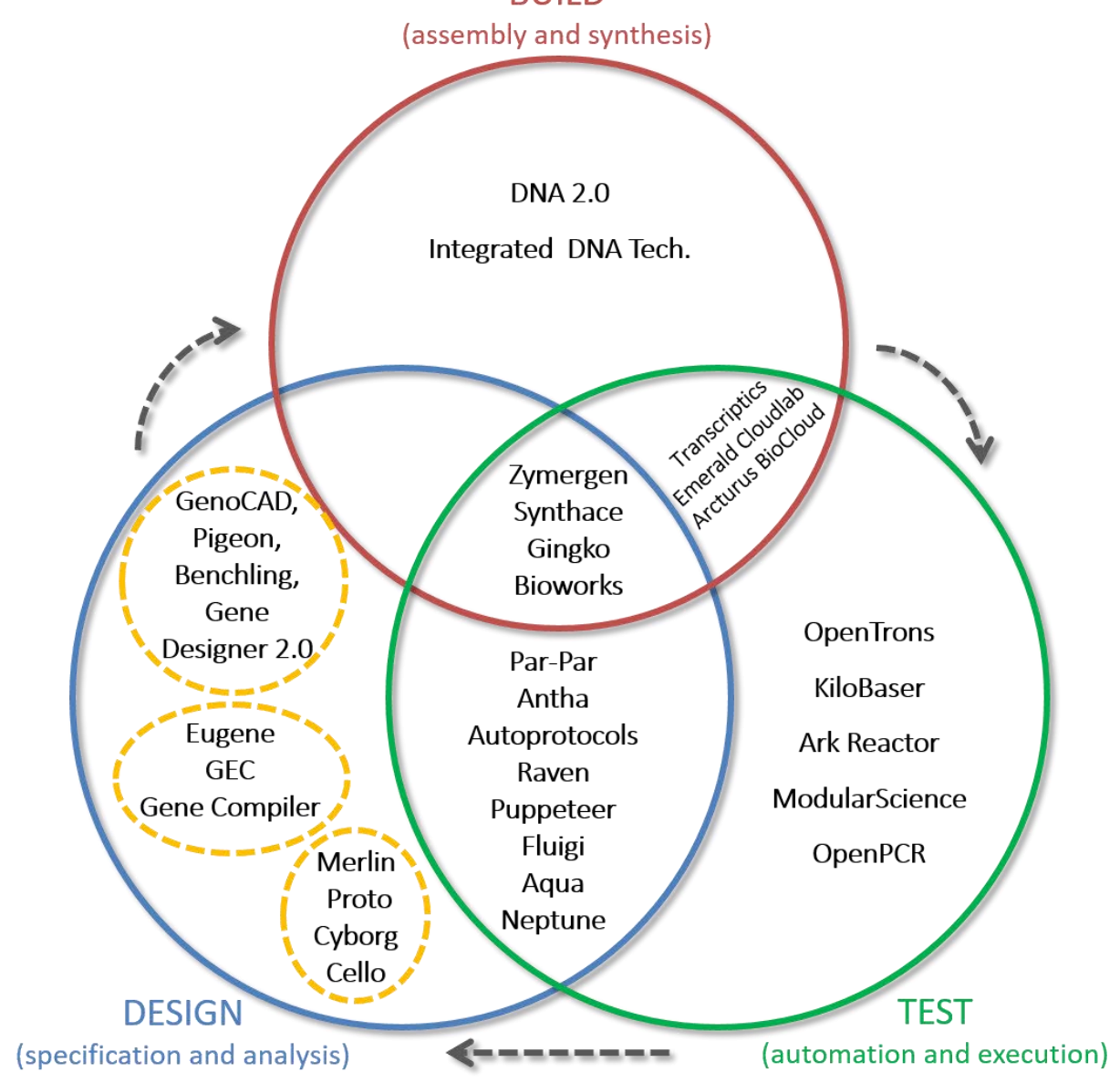

Figure 2 Classification of academic and commercial contributions in the field of automation: An attempt has been made to show the contributions according to their functions by placing them in the BUILD, TEST and/or DESIGN part. They yellow dotted circles in the Design section further classify the tools based on their function i.e. if they are DNA level (top yellow) specification tools, Function level (middle yellow) or System Level (bottom yellow).

\subsection{TEST}

The testing part of the cycle is usually carried out in most laboratories using conventional hardware (using manual pipettes); very little has changed in the way of conducting experiments in past few decades. Laboratories with good funding can afford to buy expensive robots for automation to conduct experiments at high-throughput. However, in recent years there has been an increased interest in automation and many innovative and low-cost solutions have 
been proposed and launched. They can be broadly categorized into two types Bench top and Cloud Solutions. The former consists of hardware devices that are low-cost (by at least an order of magnitude), customizable, controlled with open source software and can be potentially be set up on a lab bench. Examples are OpenTrons (low cost pipetting robots for liquid transfer), Modular Science (an automated lab bench setup with common operations like liquid transfer and centrifugation), Open PCR (a low-cost PCR machine), KiloBaser (a rapid DNA synthesizer) and Ark Reactor (an open bioreactor). ${ }^{28-32}$

There has been a recent rise in development of DIY bioreactors with multiplex capabilities designed for different functionalities like Turbidostats and Chemostats ${ }^{33}$. These millifluidic devices allow long-term investigation on cell cultures. Long-term studies require cells to grow in constant state without environmental fluctuations (nutritional status) which can trigger stress pathways ${ }^{34,35}$. Turbidostat is a continuous culture device which adjusts the dilution rate of the cell culture to maintain a constant turbidity in it. Chemostats are continuous cell culture devices which maintain the cell culture by using a nutrient limiting medium to control growth rate. Klavin Lab in the University of Washington has developed a low-cost 3D printable Continuous culture device which can function as a Chemostat and Turbidostat ${ }^{36}$. Another open source bioreactor by Baliga lab can multiplex up to 16 reactors in different modes i.e. Turbidostat, Chemostat, serial dilution and batch modes. There are also other continuous culture devices developed by different groups which can function in different modes that is Turbidostat and Chemostat modes; the Versatile Continuous culture device(VCCD), Multistats ${ }^{37}$, Morbidostat ${ }^{38}$ and the Evolvinator ${ }^{39}$. They differ with each other in having different container volumes with different multiplexing capabilities. A Morbidostat apart from being used as Turbidostat or Chemostat, is primarily used to maintain a constant drug induced inhibition inside the cell culture device. It performs it by measuring the growth rate of the evolving microbial population and accordingly adjusting the drug dosage.

There are also many different microfluidic platforms available (both commercial and academic) which are being used for doing Synthetic Biology experiments. Microfluidic devices have the advantage that they require low reagent volumes, high-throughput experimentation and the 
ability to precisely manipulate very low volumes (single cell level). These devices have demonstrated to be very useful for doing DNA assembly ${ }^{40}$, purification ${ }^{41}$, cloning ${ }^{42}$ and transformation/transfection ${ }^{43,44}$. There have been many platforms developed which allow users to conduct these experiments in an integrated manner ${ }^{45}$. Some of these solutions from an automation perspective have been discussed in detail later.

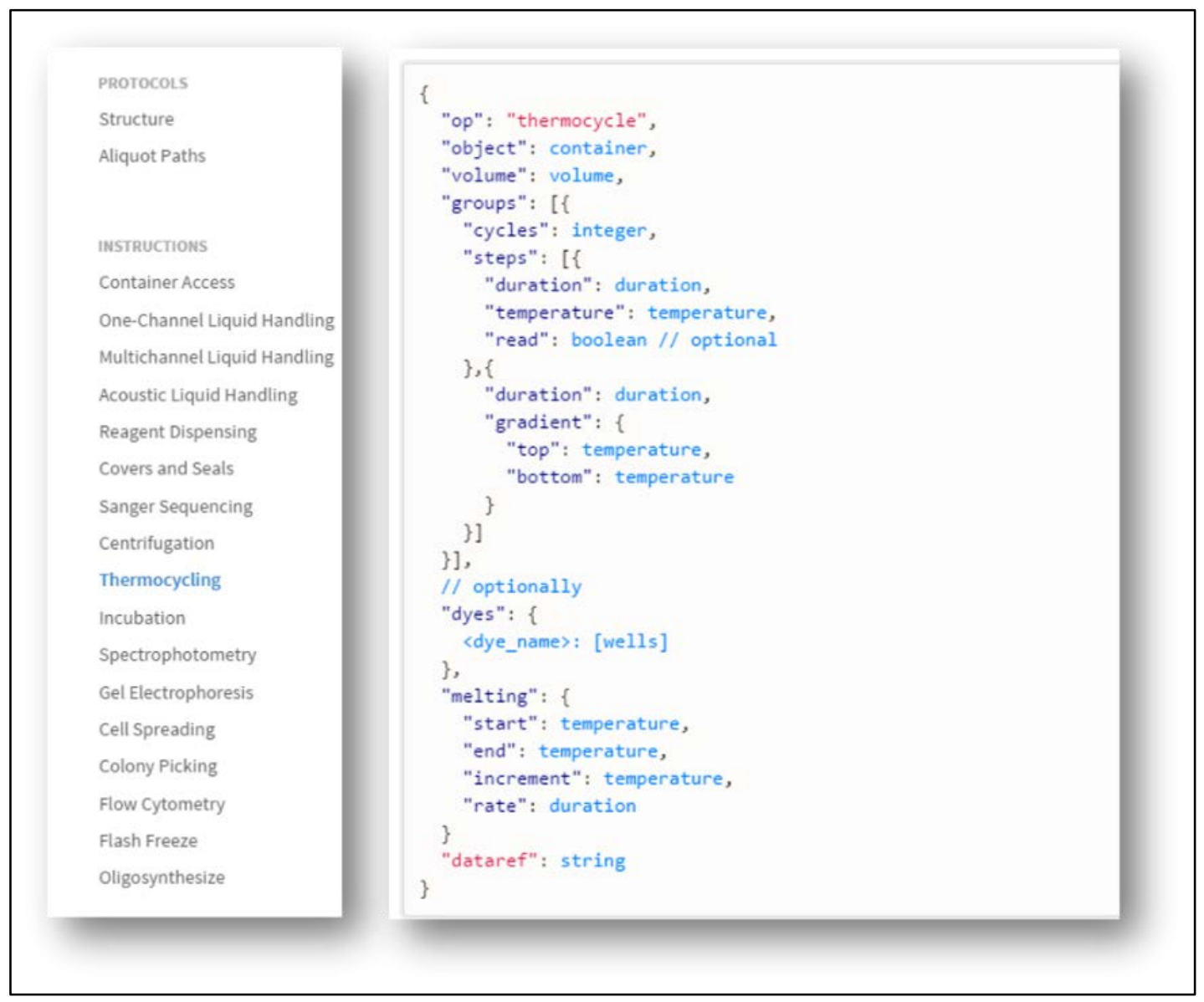

Figure 3 Autoprotocol Instructions and Syntax - Autoprotocol is a formal language to specify experiments in biology. The type of experiments that can be specified are mentioned under instructions (left). An example of a thermocycling experiment using the Autoprotocol syntax (Right) ${ }^{46}$.

Cloud Solutions consist of automated equipment pipelines platforms which to do highthroughput research. The user can mail their samples to these companies and the experiments to be conducted on the samples can be described over the internet. The experiments are 
executed in an automated fashion with minimum human intervention and the collected data sent back for analysis. Examples of some companies in this domain are Emerald Cloud lab ${ }^{47}$ and Transcriptic $^{48}$. Transcriptic uses Autoprotocol ${ }^{46}$ which is an open-standard language for specifying experimental protocols for biological research. Autoprotocol was originally developed at Transcriptic to define experiments that could be run over the internet on remote robotic automation, with the aim of moving research into the "cloud". It does not require biological knowledge to code the protocols. It is written in JSON format and is platform independent. The language is a linear set of instructions and contains no branching logic or feedback. A basic protocol defined in Autoprotocol has two segments, a set of containers (called refs) that are used in the protocol and the operations (called instructions) that are performed.

Antha ${ }^{49}$ is another solution which connects the 'design' and 'test' parts of the cycle but is not on a cloud platform. Antha is a high-level programming language for research in biology including experimental design, execution of protocols and data processing. Antha is a LIMS (Laboratory Information Management System), data-management system and Quality Assurance (QA) system designed to make workflows by stacking reusable elements called Antha elements. Elements can be genetic entities (promoter, gene, transcription factor or cells) or experimental procedures (DNA or enzyme assays). The elements can be executed in parallel in an automated manner. The language is device independent and hence the elements can be executed by hand as well. Elements can be tested, shared or embedded as a downloadable and executable material and methods section of a research article. It tracks and logs all associated data when an element is executed. It supports incorporation of quality standards such as quality by design and GLP (Good Laboratory Practice) level quality standards.

There are notable mentions which cannot be categorized in the design, build and test cycles as they overlap and fall in more than one part of the cycle. There are companies like Synthace ${ }^{50}$, Gingko Bioworks ${ }^{51}$ and Zymergen ${ }^{52}$ which are in a domain called 'microbial foundries'. Today, microbes are being used to produce novel compounds and are potential alternative to chemical synthesis. However, chemical production using microbes is a difficult task owing to complexity 
of the microbes and metabolic pathways needed to be engineered. The companies use automation, multi-variate analysis and machine learning for strain (microbe) and product optimization. They have their inbuilt software solutions which use the same design, build and test cycles. Project Cyborg ${ }^{53}$ by Autodesk, is an unreleased tool for designing synthetic biology experiments, modeling and simulations, to make 4D patterned structures i.e. structures that change with time.

In the entire Design, Test and Build cycle of Synthetic Biology there is a lack of tools which help in specification of experiment methods. Most hardware tools come with their own specification interface or programming language which is specific to the platform on which the protocol is being executed. However, there have been attempts to make general purpose programming languages for allowing user to unambiguously specify their experimental methods. Some notable examples are:

BioStream $^{54}$ was one of the first efforts in the field of computer science for the description of biological protocols. It was developed in conjunction with a Fluidic ISA (Instruction Set Architecture) to abstract the protocol description language from the fluidic hardware. An ISA works as an interface between the software and hardware. It helps to decouple/insulate the development of the software from the changes on the hardware while prototyping. BioStream is a Java library which consists of a BioStream Library to represent the fluids on the chip using First Class Objects (see Figure 4). 


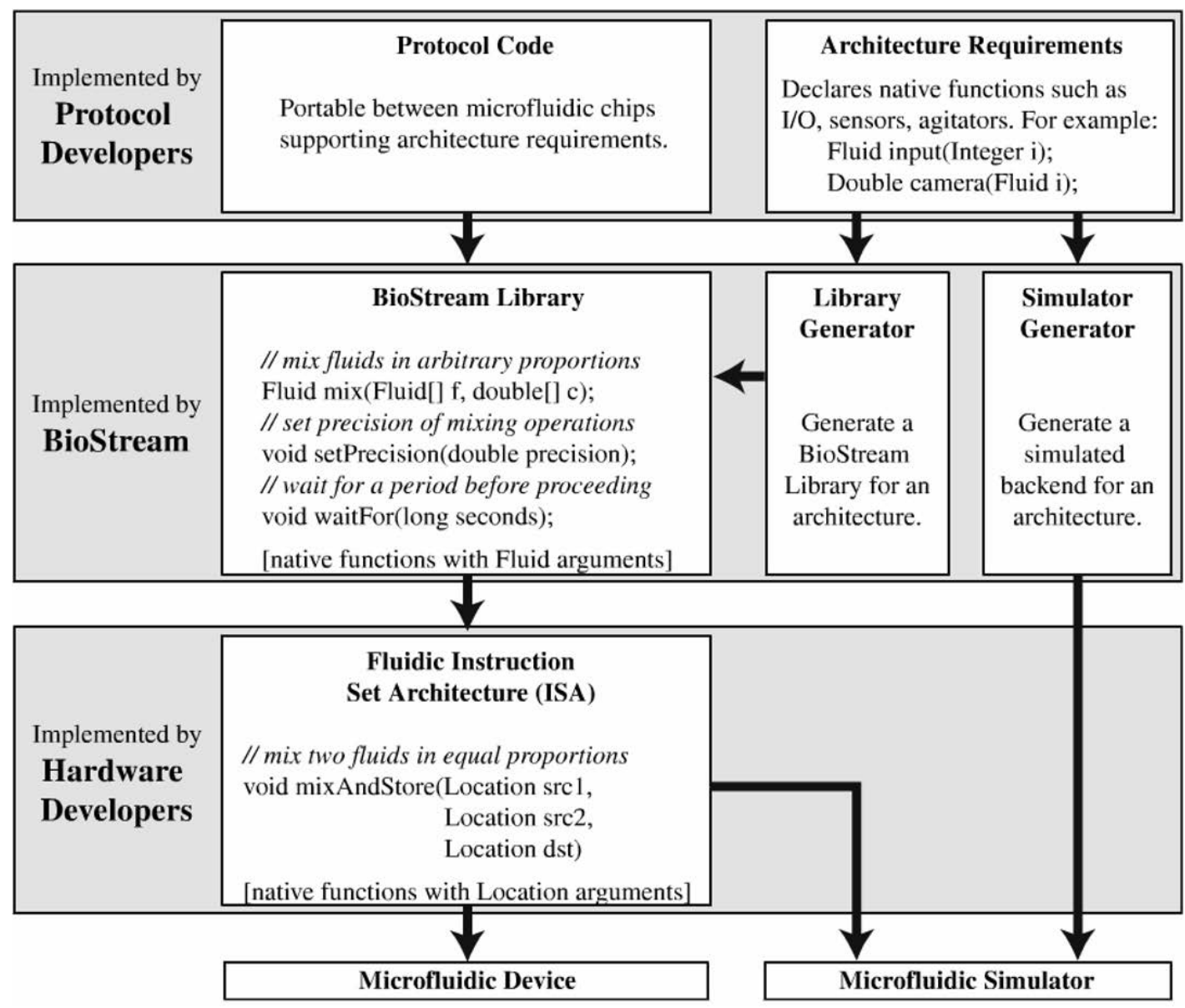

Figure 4 Abstraction layers in BioStream - BioStream required both biologists for protocol description and hardware developers for protocol execution. The BioStream library translates the protocol in BioStream to the Fluidic Instruction Set Architecture ${ }^{54}$.

Biocoder $^{55}$ is a textual high-level language for biological protocol description. It has an objectoriented architecture, based on C++. It is a platform agnostic language which allows eventual automation on a hardware platform provided intermediate layers for transforming the protocol are available. To write a program in Biocoder the user needs to be well versed in $\mathrm{C}++$. Biocoder generates a Natural Language Translation of the protocols and a graphical output called the Task Graph (see Figure 5). Even though, Biocoder was a very good attempt at removing ambiguity in protocol description using programming languages it did not automate any protocols because it stopped from generating the low-level instructions needed to control the 
actuators. Also, it did not take into consideration that the tool would be very difficult to use for people without a programming background.
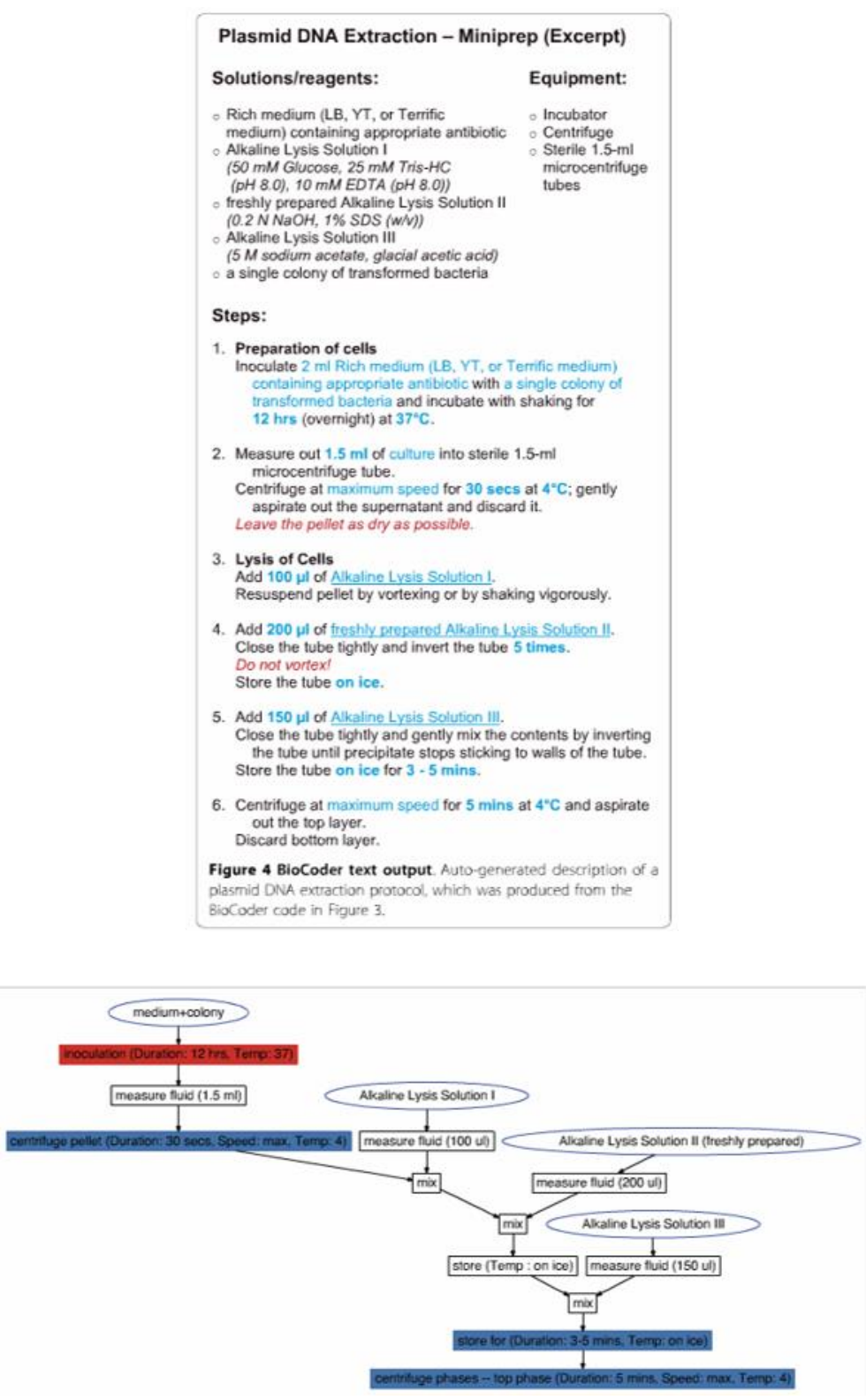

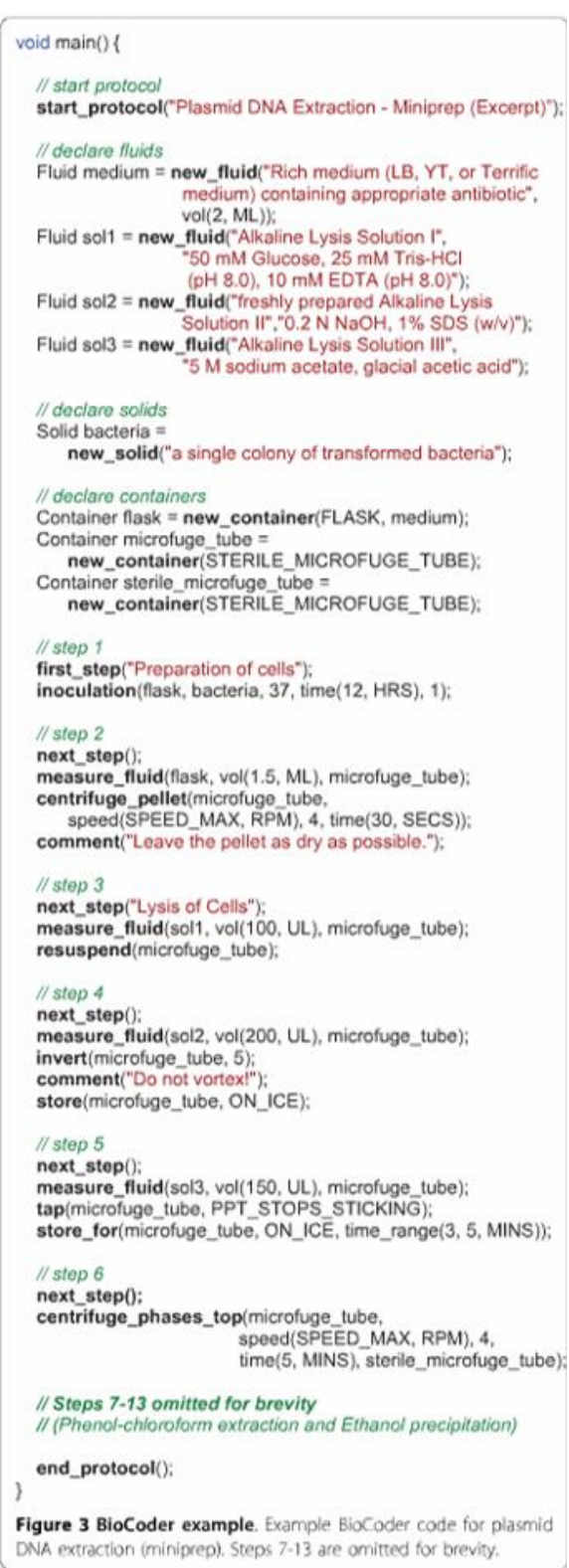

Figure 5 Different outputs of Biocoder - A protocol of plasmid Extraction in Biocoder written in C (right) is translated to English Output (top left) and a task graph (bottom left) ${ }^{55}$. 
EXACT ${ }^{14}$ is an ontology for experimental protocol description. It contains the necessary elements to describe the main elements of an experiment, but lacks sequential information which is useful when an experiment is being described (provided it is an ontology, so it's mainly used for storage and retrieve of information from databases). On the other hand, it has the advantage of being proposed as a standard way of representing experiments. Also, till date there has been no compilation efforts from this language to microfluidic hardware or robotic platforms.

PaR-PaR ${ }^{56}$ (Programming a Robot) is a high-level language for biological protocol description. It has an object-oriented approach that allows describing laboratory consumables, equipment etc. along with their properties. The main purpose of this language is the automation of biological protocols via the use of different robotic platforms. The user needs to understand all the details of the experimental setup and know the PaR-PaR language with some basic programming background. Par-Par has two interfaces a web-based mode and a command line mode. Originally designed for robotic platforms it has also been developed later for performing operations in a specific microfluidic platform ${ }^{45}$.

Clotho IDE ${ }^{22}$ is an Integrated development environment for the design and representation of genetic devices. It provides tools for accessing databases of genetic parts, defining new genetic parts (and integrating them into the database), and describing properties of these parts. It is organized as a framework which consists of application that perform specific operations. Some of the relevant tools to experiment automation offered by Clotho IDE are Assembly planner, Puppeteer ${ }^{57}$ and Puppetshow. The Assembly planner automatically plans the protocol for assembling of some specific genetic device, using previously defined parts that are stored in a gene-part database. Puppeteer is a high-level language implemented in the Clotho IDE through the Puppetshow application. This language can describe biological protocols, including calls to previously described protocols in the same language (i.e. providing modularity). The Puppetshow application is linked to the Assembly Planner application so assembly plans for the chosen biological devices is automatically achieved. In the whole process, instructions from 
Puppeteer are translated to a robot language (CRIS, Common Robot Instruction Set) to automatically execute the biological protocol via a robot.

There has also been development of programming languages and end-to-end automation solution at the microfluidic level. Some notable examples are:

Fluigi $^{58}$ is a CAD (Computer Aided Design) framework for designing microfluidic devices with a focus on Synthetic Biology experimentation. It allows the user to produce a microfluidic design from a set of specifications. In this tool, fluid units are treated as values of the system, and chambers act as logic gates (letting the fluid go or not in depends on if there is fluid in the input channel.

Neptune ${ }^{59}$ is an end to end automation tool for continuous flow microfluidic devices which allows users to specify, design and build entire microfluidic devices. It is part of host of other applications being built by the CIDAR lab along with MINT, Makerfluidics and Fluigi ${ }^{60}$.

Microfluidic Hardware Design Language (MHDL) Integrated development Environment (IDE) can compile from a High-level language (BioCoder) to an MHDL design and then to an assembly file able to perform the given operations into the automatically-generated MHDL file. This has been the first attempt to go from the level of biological description protocols to an assay-specific microfluidic device automatically. Despite this, no precise physical layout calculation for the produced devices have been performed yet (MHDL is just a logical specification of the device). The lack of such an automated process is the main technology gap required to achieve the complete automation process. MHDL allows description of microfluidic circuits in a similar manner to description of electronic circuits (in VHDL or Verilog). The language enables the user to define components from basic elements, or from previously defined components. Wires are substituted by channels between chambers, and logic gates (and timing elements) are supplanted by chambers. A simulator is also integrated with the basic compiler which validates the design by verifying the connections between elements and the pressure inside the channels and chambers ${ }^{61,62}$. 


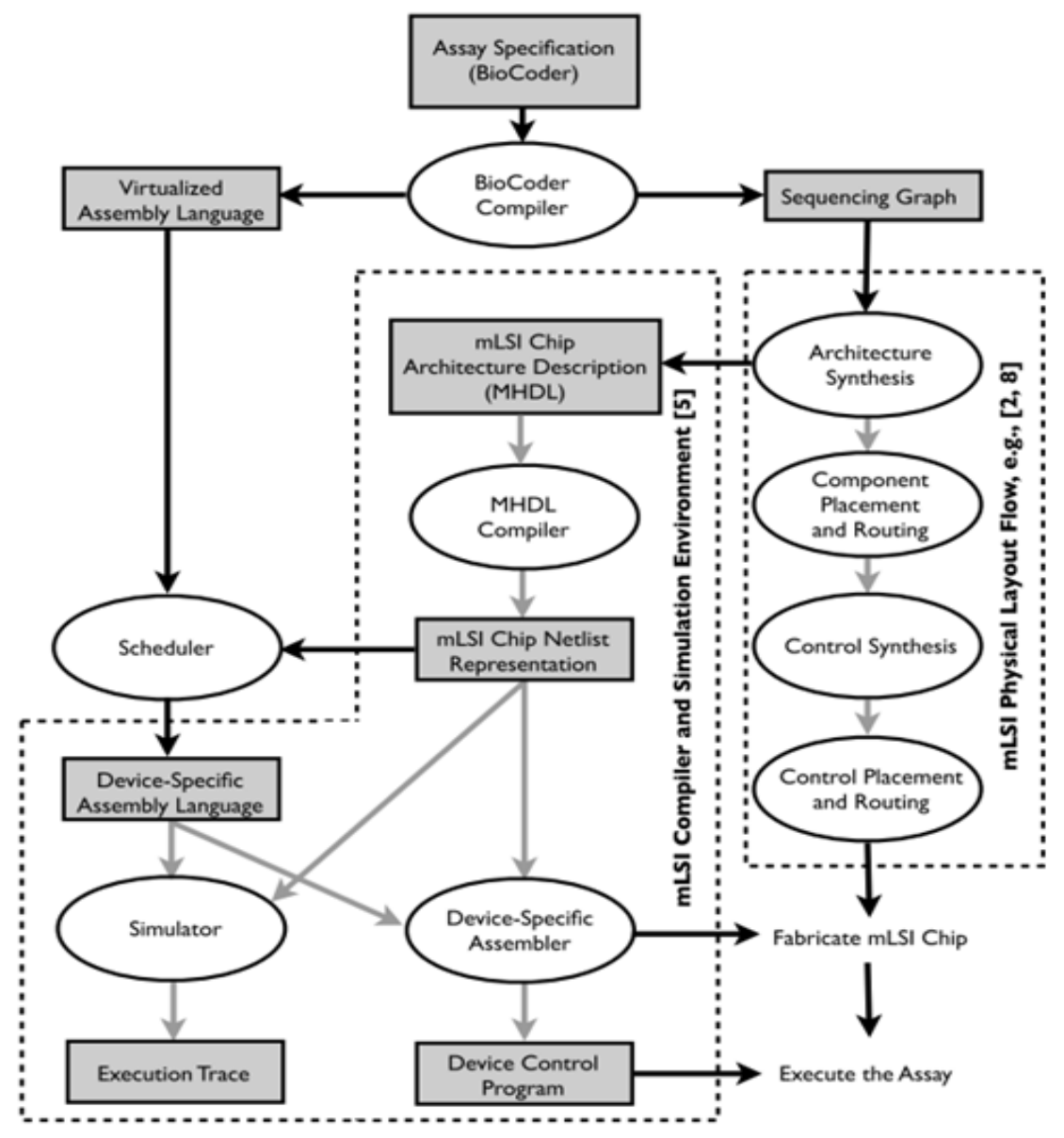

Figure 6 The software tool flow of the Microfluidic Hardware Description Language IDE - The software uses the protocol specification from Biocoder and produces a layout of the microfluidic device and the instructions that need to execute the protocol over it. It also produces a simulation result of the protocol ${ }^{62}$.

AquaCore IDE is an environment for implementing biological protocols on a microfluidic device. It allows the user to compile from a high-level language called Aqua to the low-level file specification for controlling a microfluidic device. Aqua allows description of biological experiments, in a similar way as BioCoder does (but with no object-oriented paradigm). In the process, an intermediate AquaCore file is generated. AquaCore is a language for the description of the operations inside a continuous-flow microfluidic device was developed. It was based on the idea of treating the fluid as a set of fluid units that are moved between chambers. The environment needs to be fed with the specification file of the device that is being used. This is 
the main difference with the MHDL system, which automatically generates the file with the specification of the microfluidic device ${ }^{63,64}$. 


\section{PROBLEM DEFINITION}

\subsection{Motivation}

One of the pressing concerns today is to be able to make research reproducible. Solving the reproducibility crisis would immensely help accelerate scientific progress. Now, there are many factors which lead to non-reproducible research; factors like biological complexity, lack of metrology, lack of standards (ontologies and languages) have all been discussed in detail in the state of the art. The shift of biological sciences from a qualitative to a quantitative field only exacerbates the problem. Systems and Synthetic Biology are relatively new domains in biological sciences which are fundamentally very quantitative. One of the promising approaches to solve the reproducibility problems in these domains is the use automation in the design, build and test cycles. There are many solutions (see State of the Art) which help the user automate part or fully the design, build and test workflow, but they are very specific. They can be specific in terms of their design functionality i.e. they help in automation at the level of designing functional sequences, or they can be specific in terms of building them i.e. automation tools which help in assembly or synthesis of functional sequences using particular methods (golden gate, Gibson) or they can be specific in terms of platforms they are tested on i.e. milifluidic, microfluidic devices (continuous flow and Electrowetting on Droplet platforms) or robotic platforms.

This work focusses on solving the problem of reproducibility with automation as an approach on Test module of the design build and test cycle of Synthetic Biology. There are many requirements which need to be fulfilled for the automated deployment and implementation of biology protocols on different hardware platforms. The two primary requirements are: an unambiguous language to describe the protocol which can be understood by the machine and a software which adapts the described protocol to be executed on the specific hardware platform. There are several languages available capable to describe biological protocols (requirement 1 ) but the number of languages ready to be deployed and executed in generic platforms are more reduced (requirement 2). 
The major problem with current solutions which allow users to describe their protocols unambiguously are that they are programming languages. This requires the users to learn programming which can be a burden on the user if they do not have programming knowledge. Even though, the languages are high-level in nature and have been customized to the experimental biology domain, coding in these languages can be a daunting task for a noncomputer scientist as they need to learn syntaxes, installing the software tools, in some cases even build and compile the scripts they need to use. Graphical user Interfaces (GUI) help

circumvent the programming bottleneck as they remove the need for the user to learn programing for specification of protocols. Users can in a very short time start programming their protocols on a given platform. They also provide an end to end solution i.e. from specification to execution of protocols. However, GUI come with their own set of problems. GUI in general are very platform specific which means that they are tied to a certain hardware platform. Once, a protocol is described in a GUI it can executed usually only the hardware platform specific to the GUI. There are some solutions mentioned earlier which allow users to execute their specified protocols on different configurations of a certain hardware platform but they do not allow users to migrate between different platforms or solutions. Also, protocols specified in GUls cannot be shared between users unless they are using the same GUI and compatible hardware platforms.

\subsection{RESEARCH QUESTIONS}

There is a big gap in automation for a platform agnostic end to end tool, that is specification and execution of a protocol. Users should ideally be allowed to specify their protocols in a platform agnostic way and then execute their protocols on a hardware platform of their choice. These criteria need to be met keeping in mind that user has no or minimum programming knowledge, the protocols specified should be easily shared in the community while improving the reproducibility of science. 


\section{Research Question 1:}

Is it possible to develop a high-level language for specifying platform-agnostic protocols in experimental biology which requires no programming knowledge?

\section{Research Question 2:}

Can an end-to end solution be developed which allows the execution of a platform-agnostic specification of a protocol, on a compatible hardware platform of the user's choice?

To build the ideal solution we need to highly draw upon from the concepts in computer science and other engineering disciplines. Concepts like ease of use, abstraction, modularity, interoperability and open source need to be exploited to attain our objective of building an end to end solution.

\subsection{Methodology}

The initial approach to solve the problems (mentioned above) was the development of a highlevel language for protocol specification. The language developed was called EvoCoder. It abstracted from the user all the low-level details such as the working of the fluidic hardware and electronic components. The language was based on $\mathrm{C}++$ and consisted of a simple library (collection) of primitives (commands) that are based on fluid manipulation like move, mix etc. (See Annex for the list of EvoCoder functions). EvoCoder is platform agnostic and tailored to the domain experimental biology where the experiments fundamentally involve manipulation of fluids. EvoCoder focused on executing evolutionary protocols that could be specified using its primitives, to run on continuous culture device like Turbidostats and Chemostats.

To improve the impact the possibilities of improving the high-level language were investigated. The main problem to the widespread adaptation of the software libraries was that the solution required the user (biologist) to have a good working knowledge of programming and integrated development environments. EvoCoder needed to be setup using an Integrated development environment (IDE) like Eclipse. The users could write their protocols as small scripts/programs in C language. The protocols would then have to build, compiled and executed over the 
machine. Setting up the IDE and coding protocols in a formal language (like C, Java) is a daunting task for a non-computer scientist, requiring advanced knowledge in software development. The lack of programming knowledge was barrier to the successful adoption of our work. To overcome this problem the software tool was iteratively improved. The improvements resulted in the development of an end to end automation solution which is discussed in the next section. 


\section{Proposed Solution: BioBlocks Automation Framework}

In this section, we discuss the end to end automation solution called BioBlocks Automation Framework. It allows us to address the problems mentioned in the earlier section. The solution has been designed and developed keeping the following concepts of open-source, easy to use, modularity, interoperability and abstraction in mind.

\subsection{SOFTWARE ARCHITECTURE}

The software architecture plays a very important role in laying out the different functionalities of the software modules and relationships among them. The architecture design is made keeping in mind the goals set out for the software being built. Open-Source, easy to use, modularity and Interoperability were our primary objectives. To make the software easy to use we needed a Graphical user interface and a high-level of abstraction, so that users of the software tool need not be bothered by low-level details of the functioning of the software and hardware platform (mili or micro-fluidic setups). This would allow them to focus on the biology instead of learning programming and behavior of fluidic parts like pumps and valves. The software architecture has also been designed to support modularity. The software modules can be replaced to obtain different functionality (example - generating instructions for a continuous flow microfluidic device instead of a milifluidic device) and upgraded in the future for improved performance (better routing and planning algorithms).

The software architecture has been diagrammatically explained in the following figures. Different views of the architecture highlight the different functionalities.

\section{Logical View}

The logical view explains the functionality of the architecture from the end users point of view. It explains the different software tools based on what they do. Figure 7 shows the logical view of the framework. It consists of two levels, the specification level and the execution level. The specification level (top-level) consists of two modules. They are the Protocol Specification and the Machine Specification modules. The execution level consists of the module for automated 
execution of the protocol specified on a specific machine. It generates the instructions to execute the protocol on that machine.

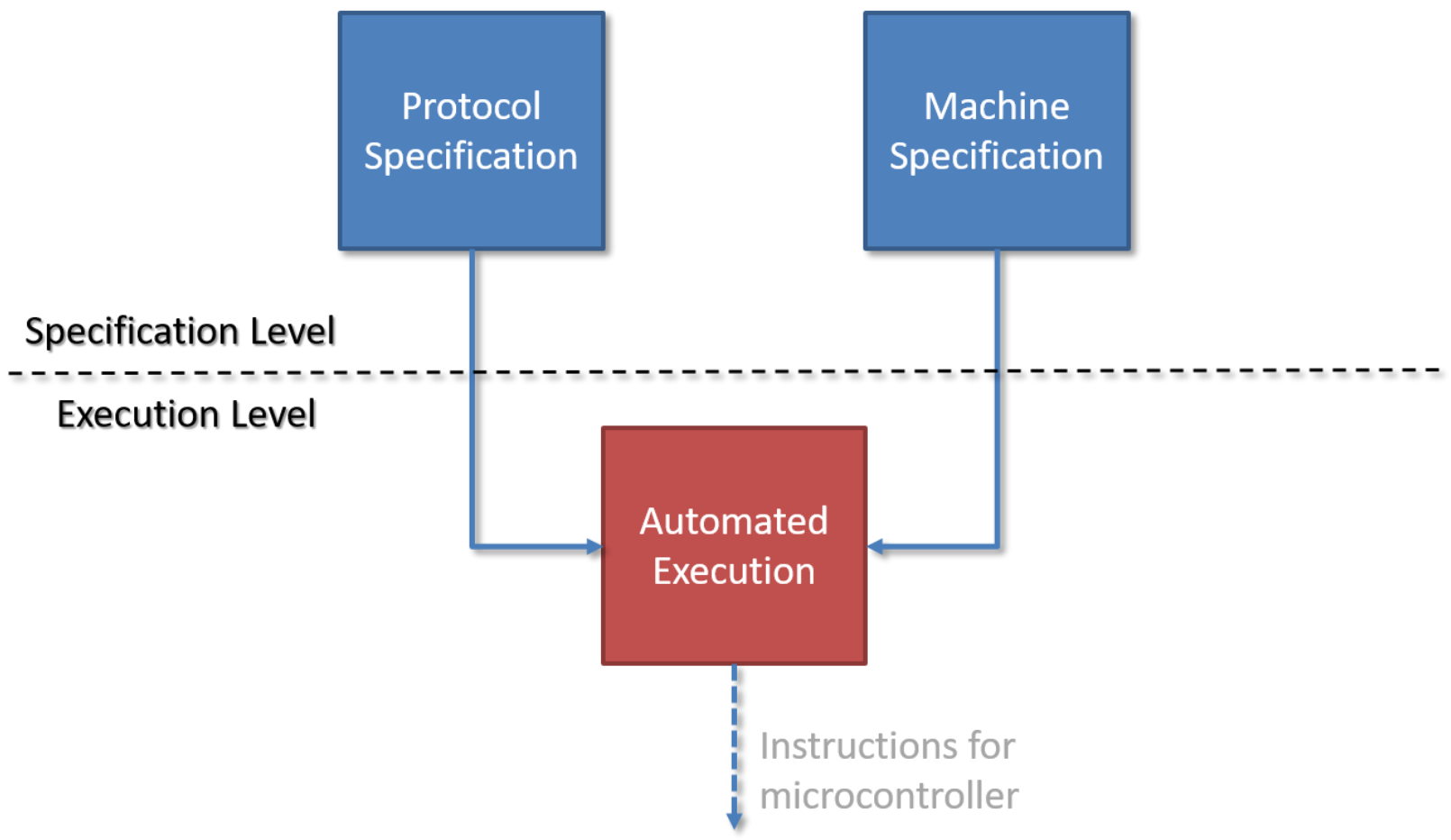

Figure 7 Logical View of the Software Architecture- The BioBlocks Framework consists of two levels; specification level for protocol and machine specification and execution level for execution of the protocol.

\section{Developer View}

The developer view explains the different modules from the software developers point of view. Figure 8 shows the developers view of the BioBlocks Automation Framework. The different modules present in the BioBlocks framework are as follows:

1) Protocol Specification GUI - For specification of protocol (1)

2) Machine Specification GUI - For specification of machine (2)

3) Protocol Translator - Translates protocol from BioBlocks to internal graph (3)

4) Machine Translator - Translates machine from machine specification to internal graph (4) 
5) Protocol-Machine compatibility check - Verifies if a specified protocol is compatible with the machine specified (5)

6) Execution Manager - Generates the machine agnostic instructions for execution of a protocol (6)

7) Plugin Manager - Generates the machine specific instructions for execution of a protocol (7)

8) Communications Manager - Communicates with the microcontroller or the simulator (8)

9) Serial Communication - Communicates with microcontroller via the serial port (9)

10) Simulator - Simulates the execution of the protocol by saving data on text files (10)

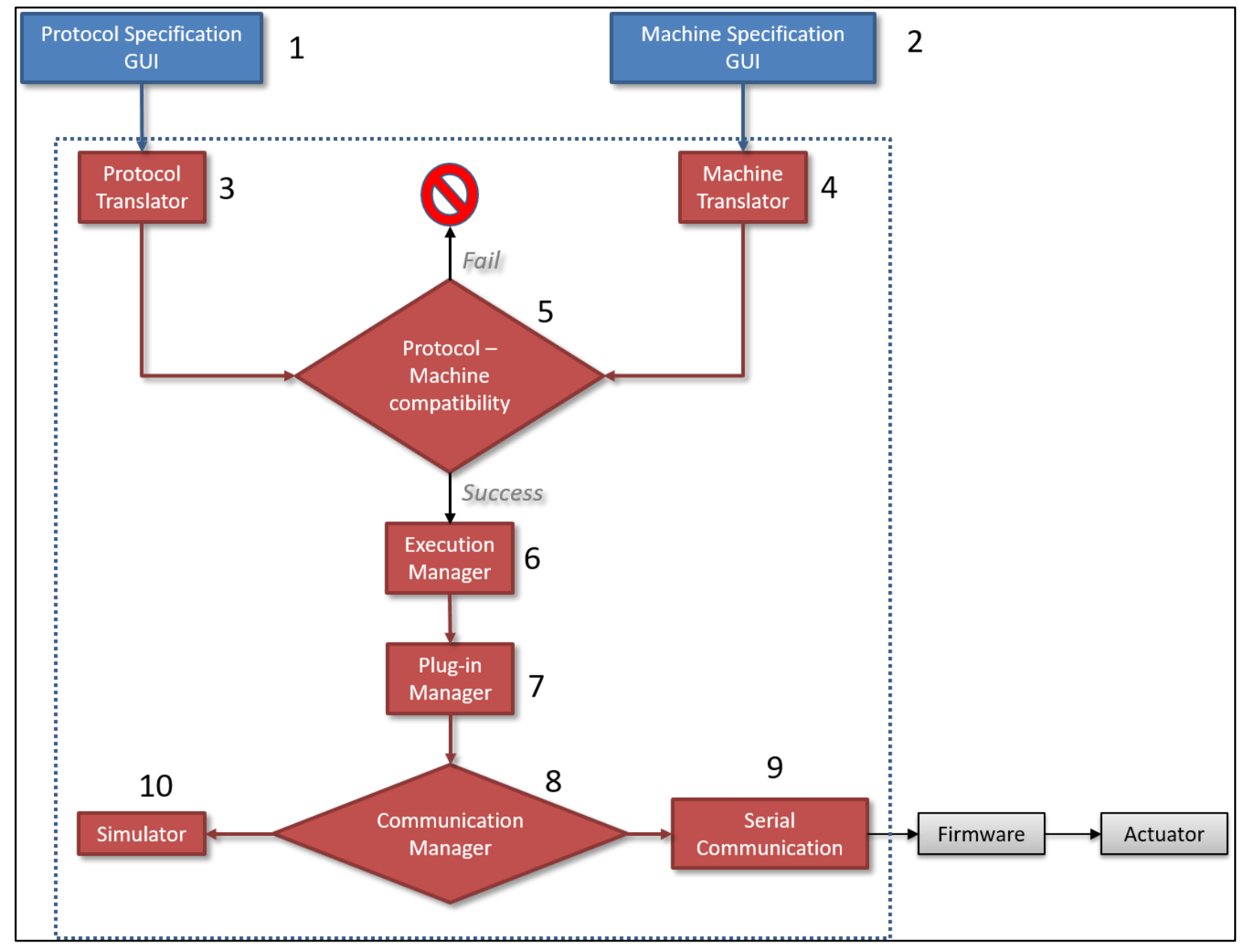

Figure 8 Developers View of the Software Architecture- The different modules developed are depicted. The Blue boxes belong to the Specification Level and the Maroon boxes belong to the Execution level. The Grey boxes are part of the Hardware. The different modules are the 
Protocol Specification GUI (1), Machine Specification GUI (2), Protocol translator (3), Machine translator (4), Protocol-Machine compatibility (5), Execution Manager (6), Plug-in Manager (7), Communications Manager (8), Serial Communication (9) and the Simulator (10).

Software tools

The BioBlocks framework is a web-based tool which can run in most internet browsers. It uses the MEAN stack which is a full stack JavaScript framework for creating web applications. M stands for Mondo DB which is a NOSQL database. The database is not being used currently. E stands for Express.js which is minimal node.js web application framework. A stands for Angular.js which is the front-end web application framework to process HTML. N stands for node.js which is the runtime environment for executing JavaScript code server-side. MEAN was chosen as the preferred stack as both Google's Blockly and Cytoscape have libraries in JavaScript.

The BioBlocks framework can be easy installed which sets up a local web-server to allow saving, sharing and local execution of protocols. It consists of a Home page with a brief description of the frame work (Figure 9). It has three other options/tabs which are also described in the logical view. It has an option to specify a protocol using the Protocol Specification App (Figure 10). It has an option to specify a machine using a Machine Specification App (Figure 11). It has an option to Execute a protocol using the Protocol Execution App (Figure 12). 


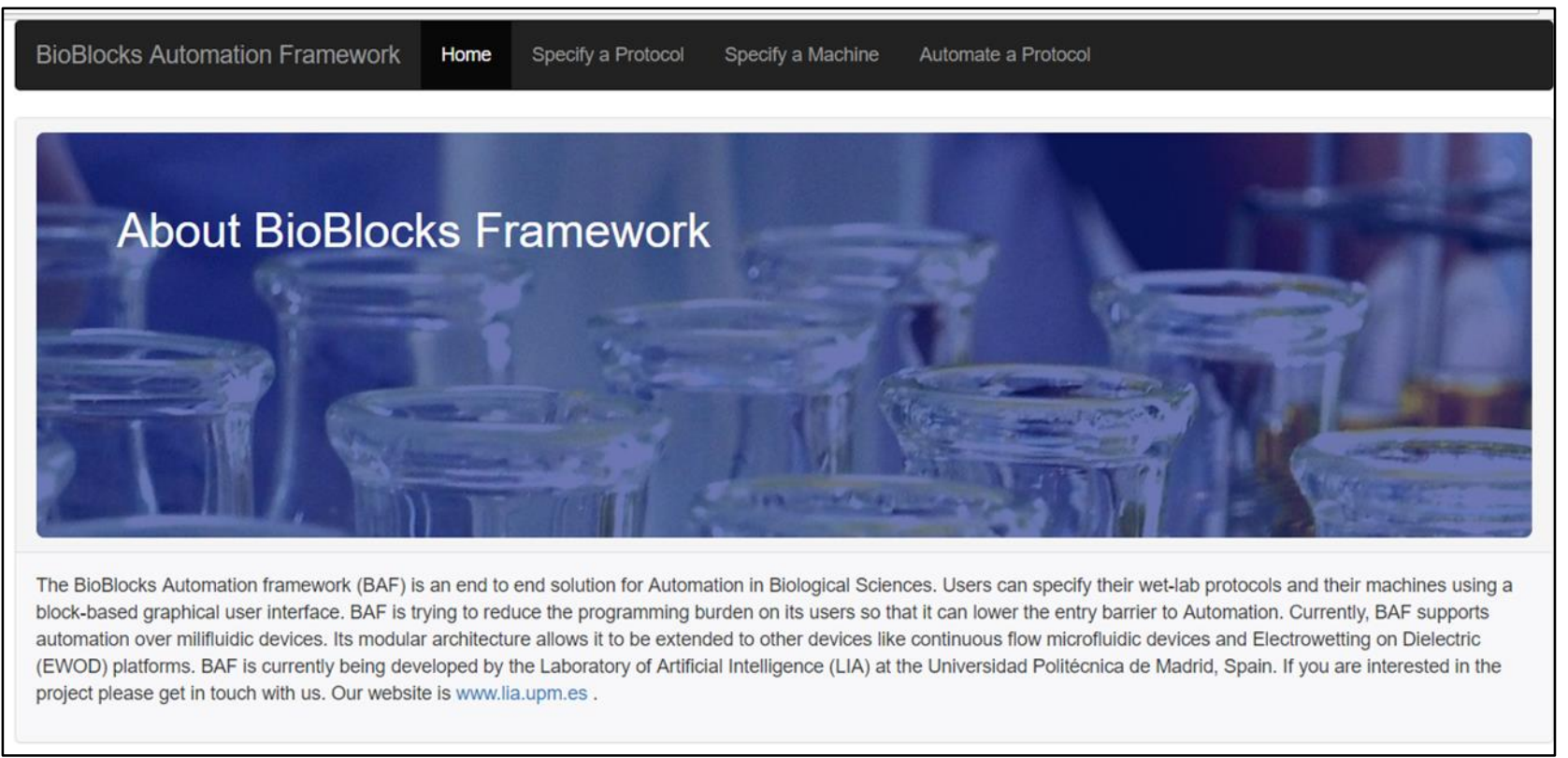

Figure 9 Screenshot of the BioBlocks Automation Framework Main Page. A brief description of the framework along with the options to 'Specify a Protocol', Specify a Machine' and 'Automate a Protocol' are present. Users can click on the tabs to access the required option.

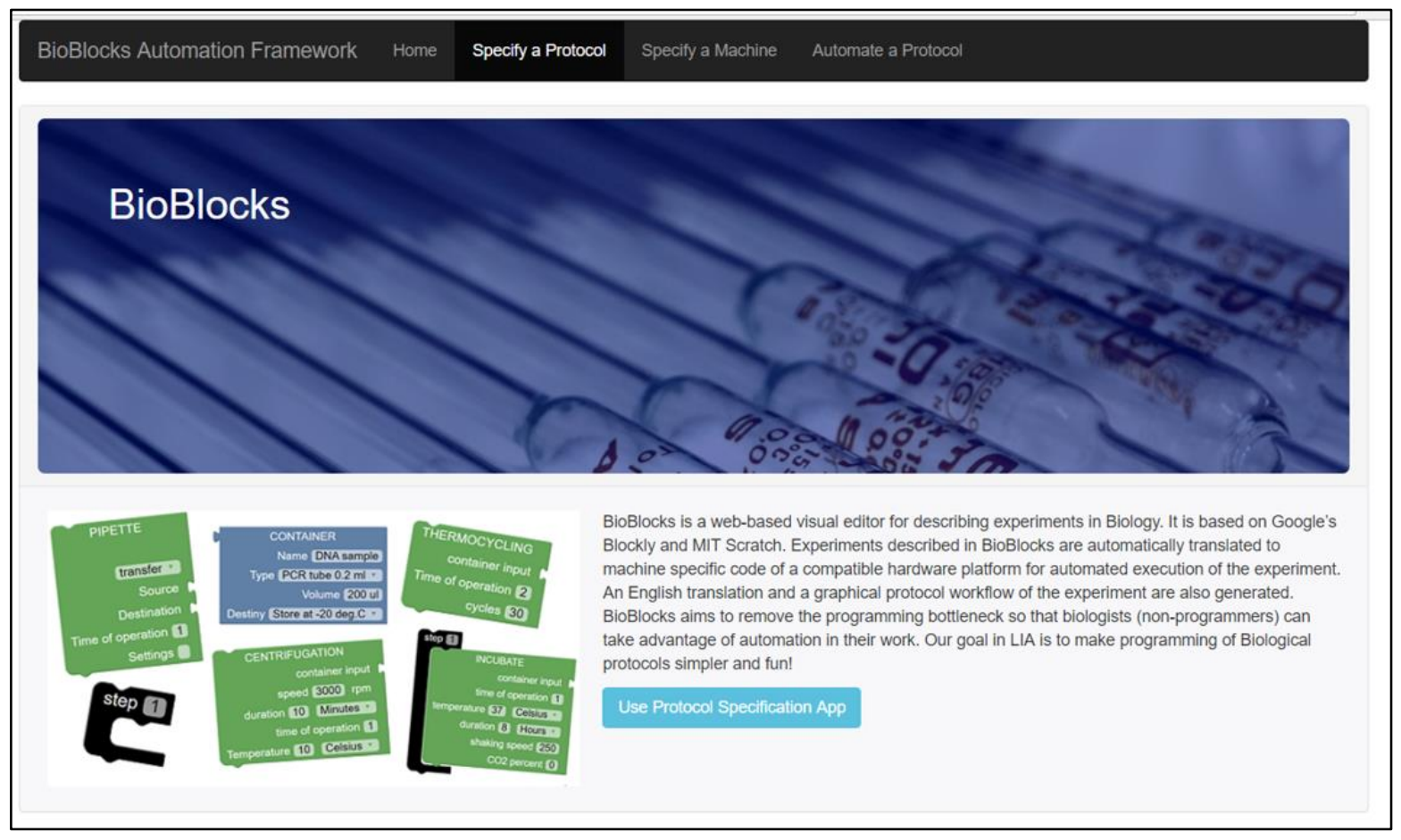


Figure 10 Screenshot of the Protocol Specification App Option in the BioBlocks Automation Framework. A brief description of the App along with a link to the App is provided. The App opens in a new browser Tab.

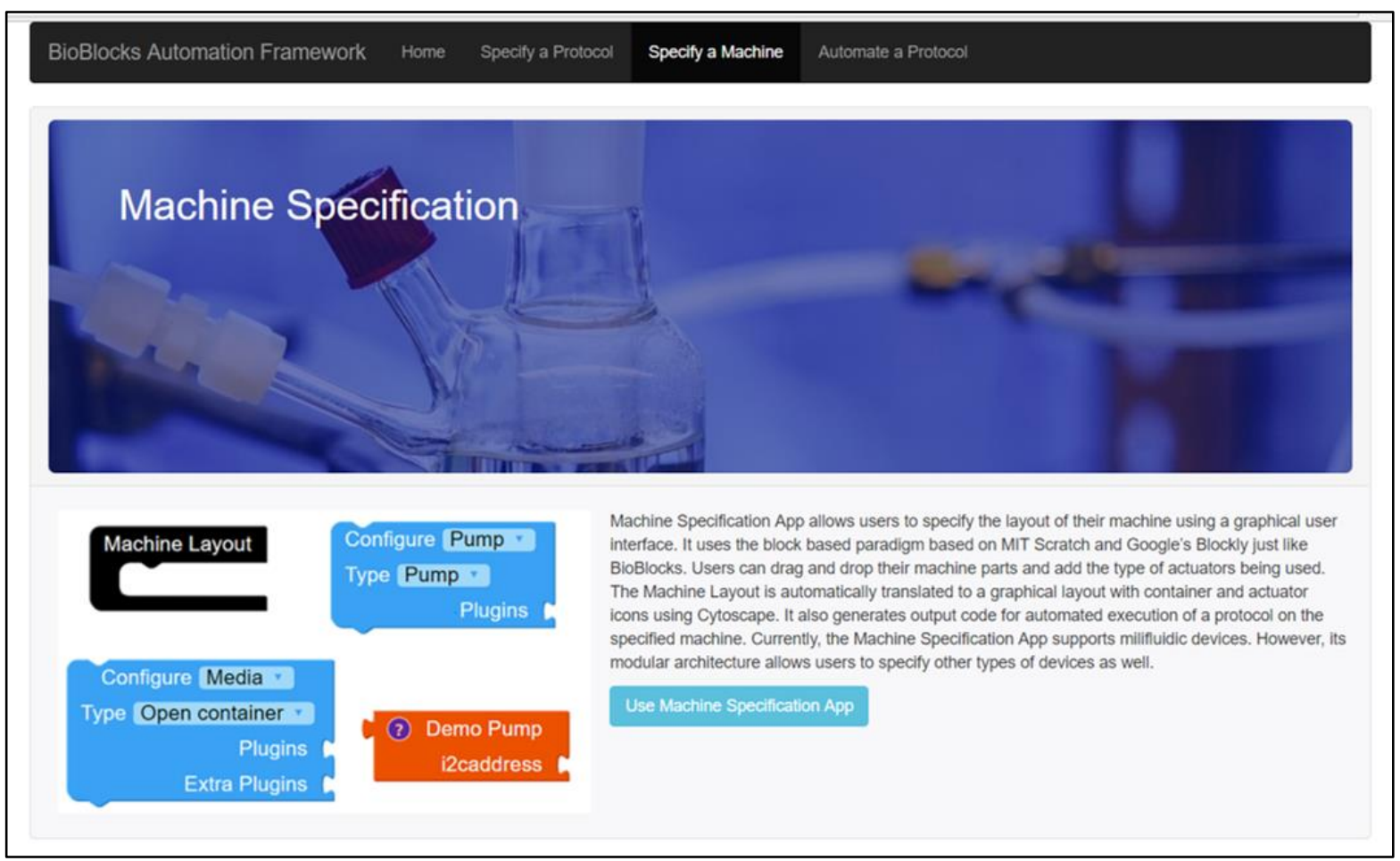

Figure 11 Screenshot of the Machine Specification App page in the BioBlocks Automation Framework. A brief description of the App along with a link to the App is provided. The App opens in a new browser Tab. 


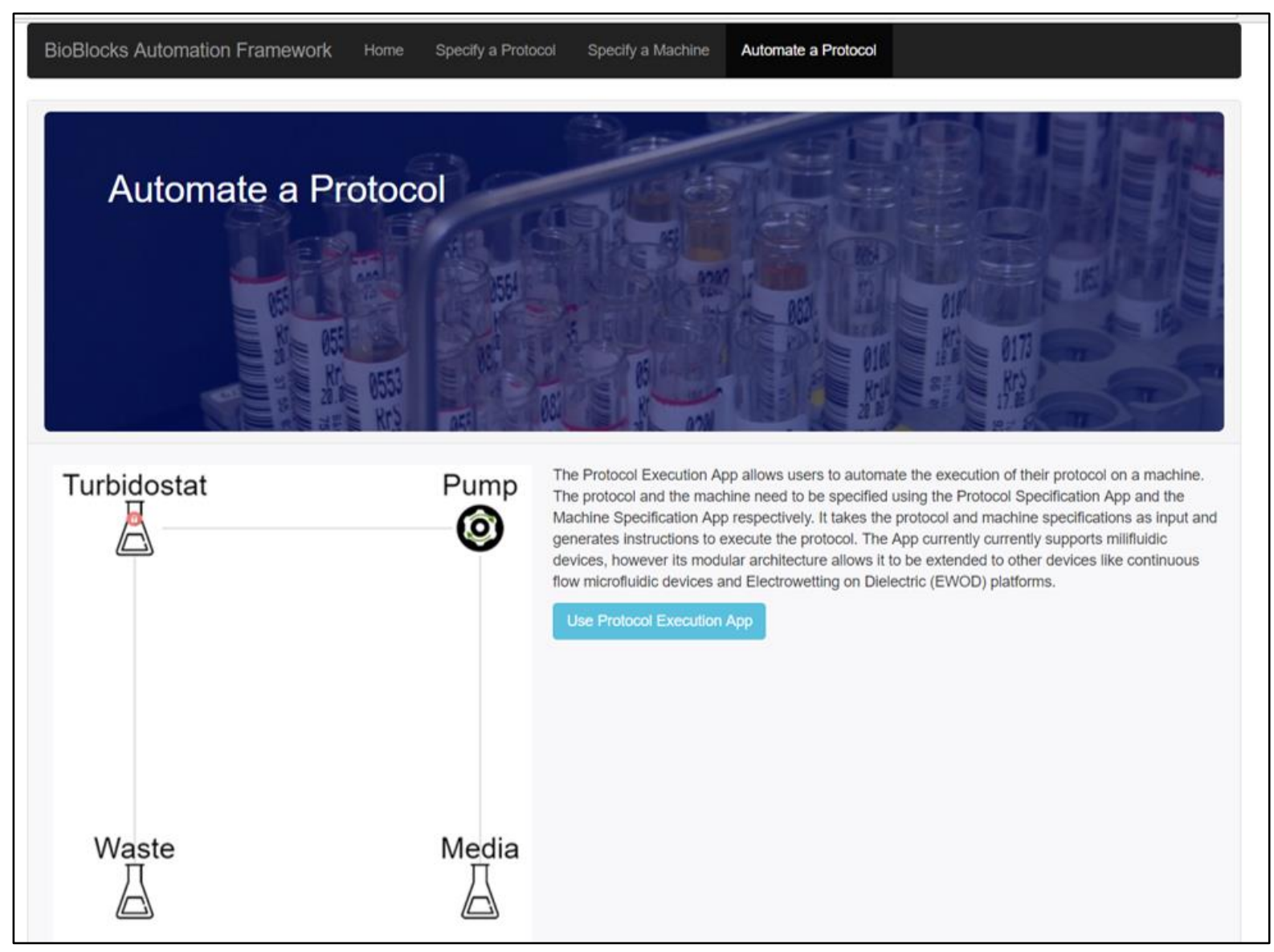

Figure 12 Screenshot of the Protocol Execution App page in the BioBlocks Automation Framework. A brief description of App along with a link to the App is provided. The App opens in a new browser Tab. 


\subsection{SPECIFICATION LEVEL}

The BioBlocks Automation Framework consists of two different types of specifications. The first specification is for specifying the protocols using the Protocol Specification App. The second specification is for specifying the machine layout using the Machine Specification App. Graphical User Interfaces (GUI) have been developed to allow users to specify the protocol and machine in a simple manner without the need to learn programming. The GUI uses the blockbased programming framework by Google called Blockly ${ }^{65}$. Blockly uses a jig-saw based paradigm: the blocks are shaped in jig-saw like pieces and compatible shapes snap fit together. The jig-saw paradigm was chosen as it is being used to teach beginners programming without the need to learn formal language specific syntaxes. This reduces the learning burden on the user and makes programming very accessible. Using the same approach, we hope that biologists will be able to specify their protocols and their machines without the necessity to learn complex language specific syntax. The users can drag and drop blocks from domain specific (Biology and Hardware) block libraries to assemble the specification of a protocol or a machine.

\subsubsection{Protocol Specification App}

The protocol specification App consists of BioBlocks ${ }^{66}$ which are a library of customizable blocks. Using the BioBlocks toolbox, experimental protocols can be described in a simple drag and drop manner. BioBlocks were developed by customizing the blocks and grammar of Blockly. The logic of BioBlocks is primarily based on Autoprotocol (insert citation), a language for specifying experimental protocols in biology. It was developed by Transcriptic, a lab-in-a-cloud company. Autoprotocol does not allow for specification of conditional protocols, therefore BioBlocks was modified to allow for conditional specification.

There are three main types of blocks available in the BioBlocks library (See Figure 13). They are the 'container blocks'(blue), the 'operation blocks'(green) and the 'organization blocks'(black). The container blocks represent the commonly used containers in wet-lab experiments. Some examples are Eppendorf tubes, multiwell plates (like 96, 384 well plates), beakers and flasks. The operation blocks represent operations that are performed on the containers. Some 
operations that are available in the BioBlocks library are transfer, measure, thermocycle, centrifuge etc. A complete list of operation can be found and the corresponding blocks can be found in the appendix [insert appendix link]. The organization blocks help the users to design and organize their experiments in a familiar manner i.e. a format like writing protocols in a lab notebook in natural language (English). An experimental protocol consists of multiple steps and the users can use the organization blocks to add steps to their protocols like Step 1..., Step 2... etc. There are a fourth type of blocks called 'conditional blocks'(orange) in the BioBlocks framework to specify conditional protocols. They have been adapted from Google Blockly native block library and modified to be used with BioBlocks.

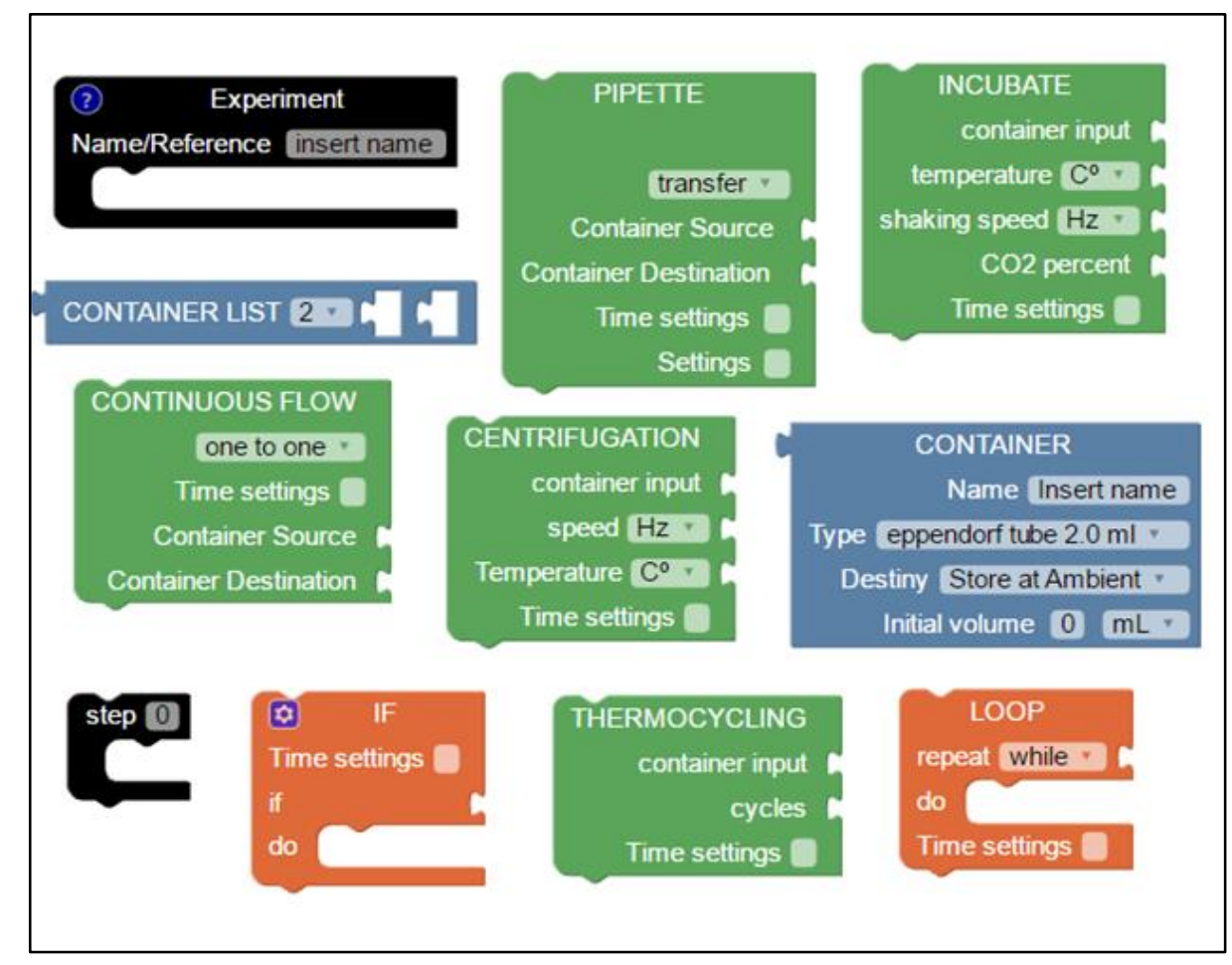

Figure 13 BioBlocks Library - A snapshot of some of the Blocks available in the BioBlocks Library. The container blocks are shown in blue, the operation blocks are shown in green and the organization blocks are shown in black. Blockly's native 'conditional blocks' (orange) have been modified to allow for specification of protocols using conditions.

The different blocks can be dragged and dropped from the BioBlocks library using the Protocol Specification App to specify a protocol (See Figure 14). In the example, a PCR (polymerase chain 
reaction) is specified with the help of 4 different Blocks. BioBlocks allows specification of complex protocols which use conditional actions. Conditional actions are operations which need to be performed depending on meeting the requirements of condition specified by a user. The condition is usually based on an input based on a measurement. The variable native block from Blockly can be specifically used for specifying such complex protocols. An example of complex protocols is shown in Figure 15.

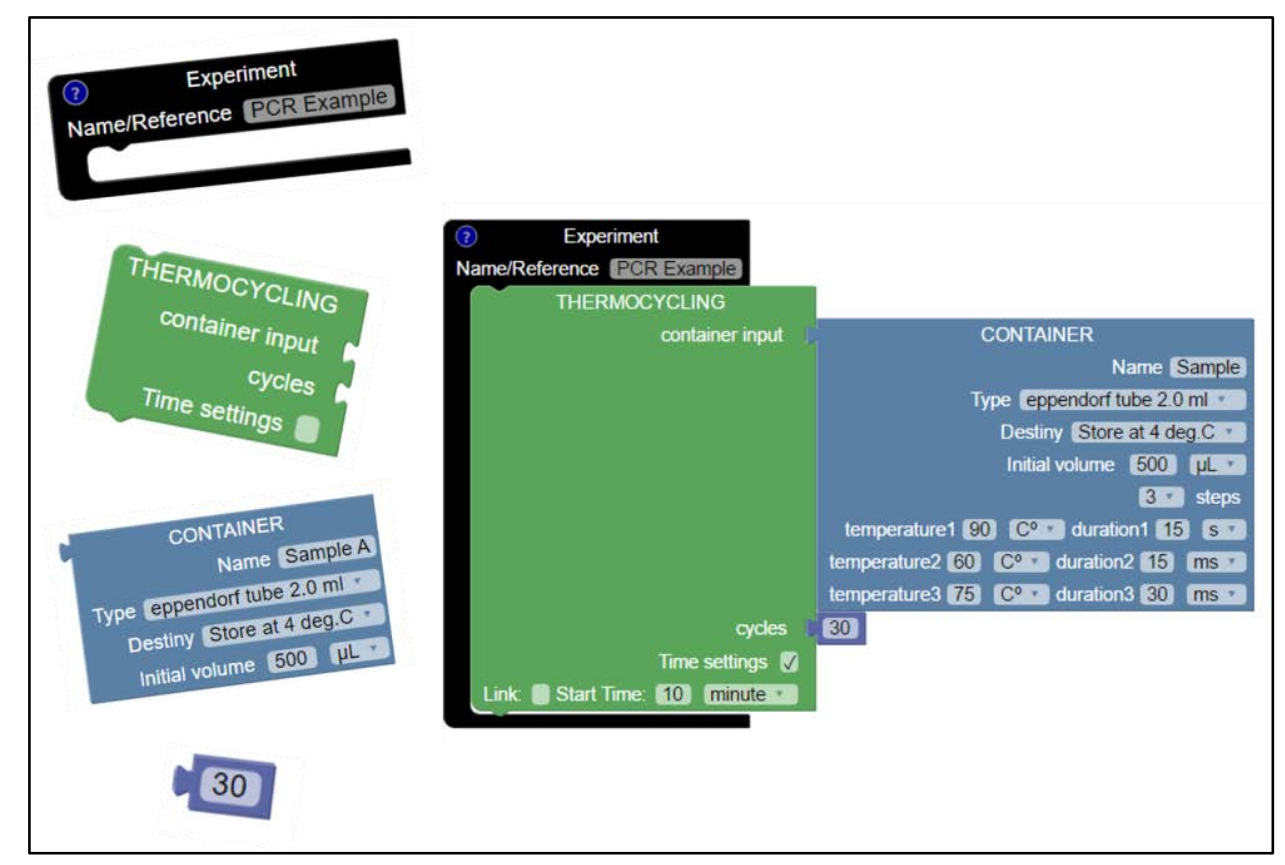

Figure 14 Protocol Specified in BioBlocks - The operation block (green), the container block (blue), the organization block (black) and the native Blockly input block (purple) on the left can be attached to each other to form a protocol (right). In the above example a PCR experiment is designed. The shapes of the block guide the user to specify a protocol in an easy manner. 


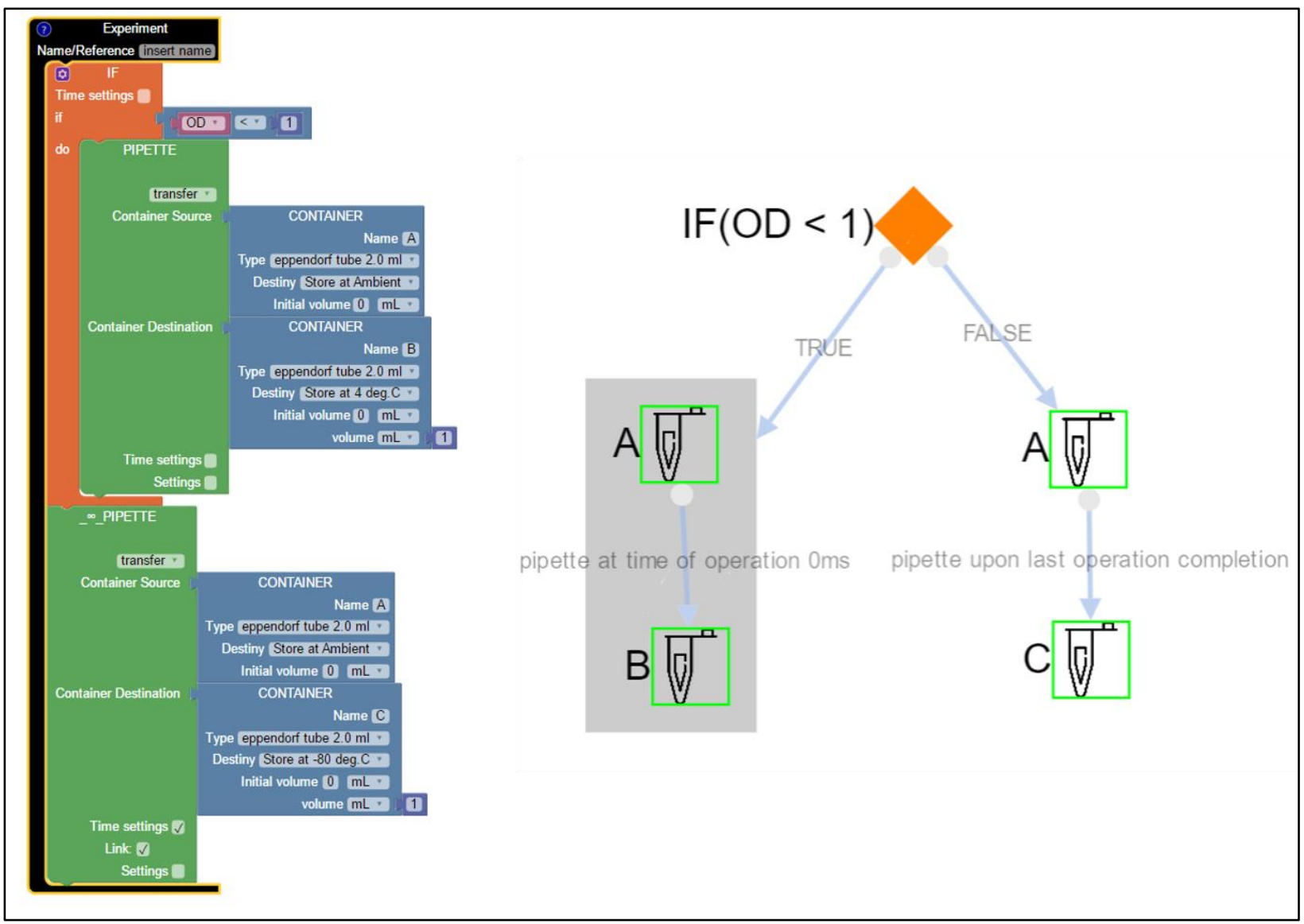

Figure 15 Specifying conditional protocols using BioBlocks - In the above example a conditional protocol based on an OD measurement has been specified (right). Depending on the OD value that IF $O D<1$, a transfer is made from Tube $A$ to Tube $B$ or from Tube $A$ to Tube $C$. The protocol workflow is equipped to represent the conditional operation which is highlighted in a grey box (right).

Blockly allows users to do not only basic conditional operations but advanced one as well. Users can clock on the settings icon of the conditional block to add more sub-conditions (see Figure 16). 


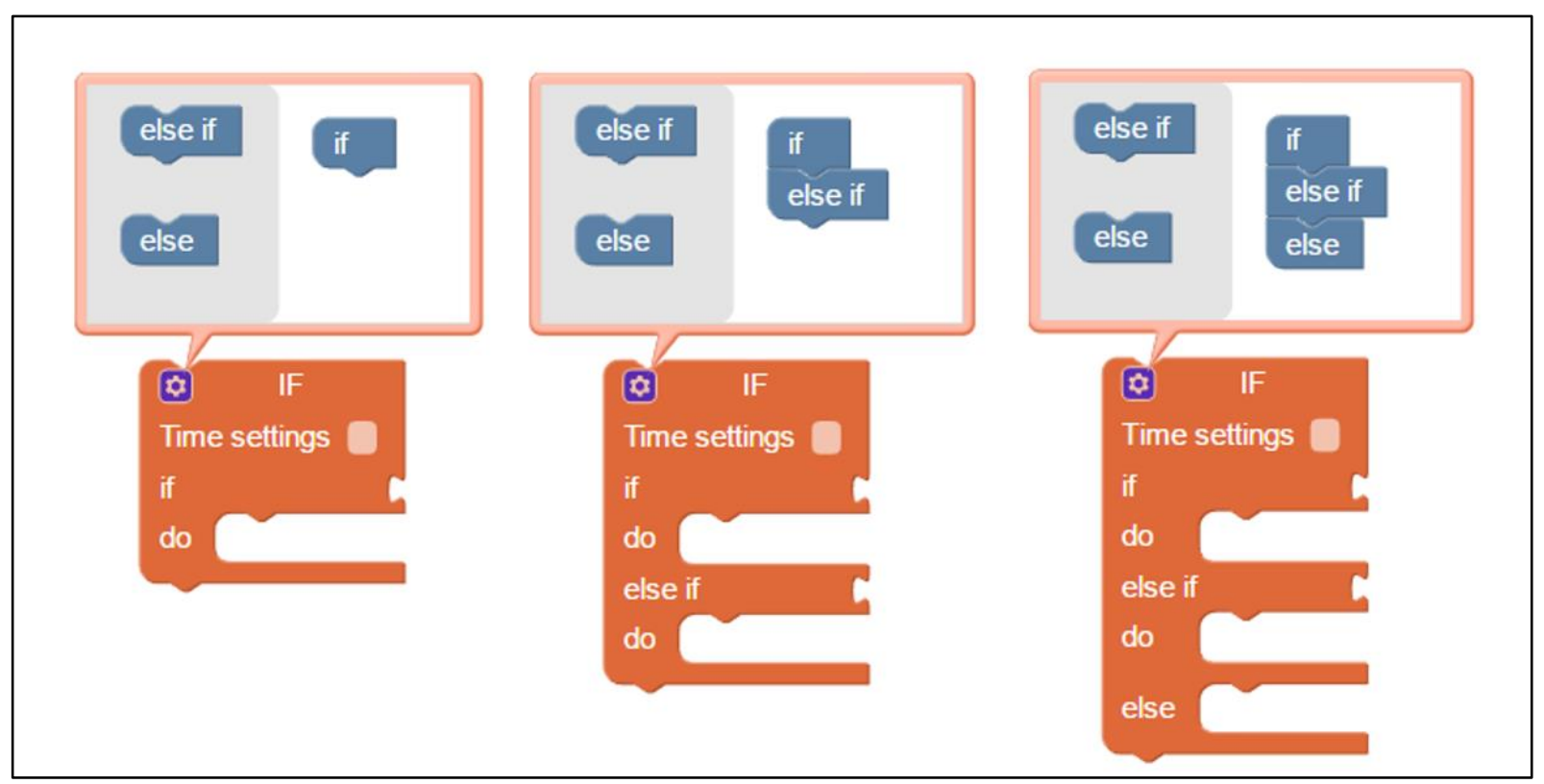

Figure 16 Advanced conditional operation specification - BioBlocks uses a very powerful native Blockly feature to allow specification of advanced conditional operations. Users can click on the settings icon and drag and drop 'else' or 'else if' blocks (blue) to add more conditions to the IF block. 


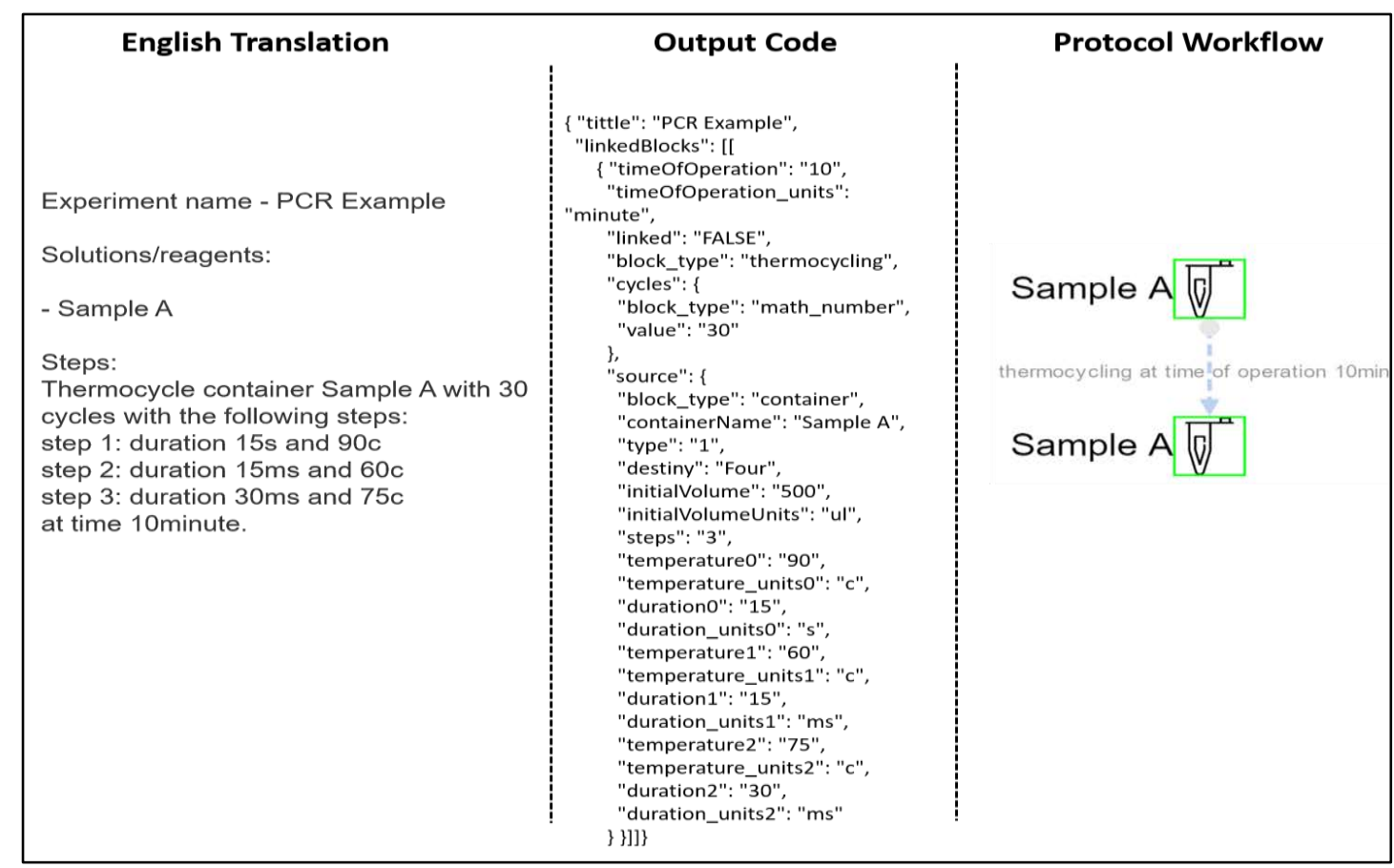

Figure 17 Multiple outputs of protocol specified in BioBlocks - The PCR example in Figure 14 generates the three outputs automatically in real-time. An English transition of the specified protocol, Output Code which is machine readable and a Protocol workflow for visual aid are generated.

\section{Multiple Outputs}

An experiment specified in BioBlocks in automatically translated into real-time to give multiple outputs.

\section{Natural Language Translation}

A protocol in BioBlocks is translated to a Natural Language (English) for easy understanding of the protocol. It also helps the user in cross-validating that the protocol specified in BioBlocks that is to check whether the protocol specified is what the user wanted. The Natural Language translation is structured in a conventional format. The format is as follows: Experiment Name, lists all the reagents used, list of all the equipment and the Steps of the conducting the experiment (See Figure 17 left). 


\section{Output Code}

BioBlocks also translates a protocol automatically to machine compatible code. Current version of BioBlocks translate the blocks to JSON code. The JSON code is largely based on Autoprotocol which is a formal language for specifying experimental protocols in Biology. It is developed by Transcriptics, a biology in the cloud laboratory. Autoprotocol is a linear set of instructions and does not allow conditional actions to be specified in protocols. To allow users of BioBlocks the ability to specify complex protocols we modified the Autoprotocol to support conditional action specification. The machine code output can be modified by the user to generate code specific to their choice of machine or robotic platform (See Figure 17 Middle).

\section{Graphical Workflow}

To assist the user in execution of the protocol, BioBlocks is also translated to generate a workflow of the protocol. The protocol is expressed a graph with nodes and edges. The containers are represented as nodes and the edges represent the action that are performed over the containers (See Figure 17 Right). To generate the graphs, Cytoscape JS library was used. Cytoscape is a very powerful data visualization tool used by bioinformaticians and systems biologists to visualize complex data sets ${ }^{67}$. We have used this tool to allow users to visualize the workflow of complex protocols. The notation of the nodes, edges and conditional actions are shown below (See Table 1). 
Table 1 Graphical Notation used in the Protocol Workflow

\begin{tabular}{|l|l|l|}
\hline Node Types & Container Nodes & \\
\hline Edge Types & Conditional Node & \\
\hline & Ciscrete Operation/ & \\
\hline & Conditional Edge & \\
\hline & Continuous & \\
\hline Box & Operation & \\
\hline
\end{tabular}

\section{Customization of Blocks}

BioBlocks is open-source in nature and this gives users the ability to customize it as per their requirements. There are various types of customization that be carried out on BioBlocks. The blocks can be modified to generate machine code compatible for their choice of robotic platforms. Blocks can be customized to introduce constraints; inhibit the linking of two 
incompatible blocks (i.e., the blocks snap away). Constraints are used in the BioBlocks design to avoid different types of errors such as syntactic and logical errors. Since the code is generated in an automated manner, when the blocks are connected, traditional syntactic errors are avoided. Further, the blocks can be customized to a specific domain to avoid domain specific syntactic error. BioBlocks have been customized to the experimental biology domain to avoid bio-syntactic errors. This is done by applying constraints which inhibits the linking of two more types of blocks. E.g. operation such as measurement of OD which are compatible only with specific types of containers (quartz cuvettes). Logical errors constitute errors like overdrawing fluids, under drawing fluids and dead volumes from a container during a transfer operation. An example of over drawing is transferring $200 \mathrm{ul}$ from a tube which contains less than 200ul of liquid. Under drawing fluid deals with the sensitivity of the equipment used for aspiration/dispensing. For example, an equipment's working range is between 200-1000ul and the user specifies to move 10ul with it. Dead volume is another important parameter which needs to be considered when doing fluid manipulation. Dead volume is the volume that cannot be aspirated/ dispensed (transfer) because of the liquid sticking to the walls of the container. In BioBlocks users can customize the different types of logical errors associated with different equipment. These constraints are encoded system-wide in the blocks. Since, BioBlocks is made open-source the users can create novel blocks with new functionalities with a different set of constraints. Hence, allowing them to create their own library of blocks for their specific platforms.

BioBlocks features for improved usability

Visual Manipulation

Special attention has been paid to allow for easy visual manipulation of specific experimental protocols. Blockly uses two different types of connectors for connecting blocks (See Figure 18). These connectors guide the user to connect only compatible blocks hence reducing the specification errors. Different block types have different colors for improved usage (See Figure 13). The blocks can be collapsed and expanded to allow for navigation through length complex protocols (See Figure 19). 


\section{Commenting}

Blocky allows users to comment on any blocks. This feature has been included in the BioBlocks framework. Users can comment on any block by right clicking on the Blocks. A pop-up window opens a text-editor where users can note their comments. These comments are programmed to appear in the English Language output. This feature can be very useful as critical steps can be commented.

Dynamic Blocks

Blockly has a very power feature called Dynamic Blocks. The inputs displayed on the Blocks are not static. They can be programmed to change based on previous inputs. This concept of progressive disclosure of input options which a user needs to fill lowers the memory burden on the user (See Figure 20 ).
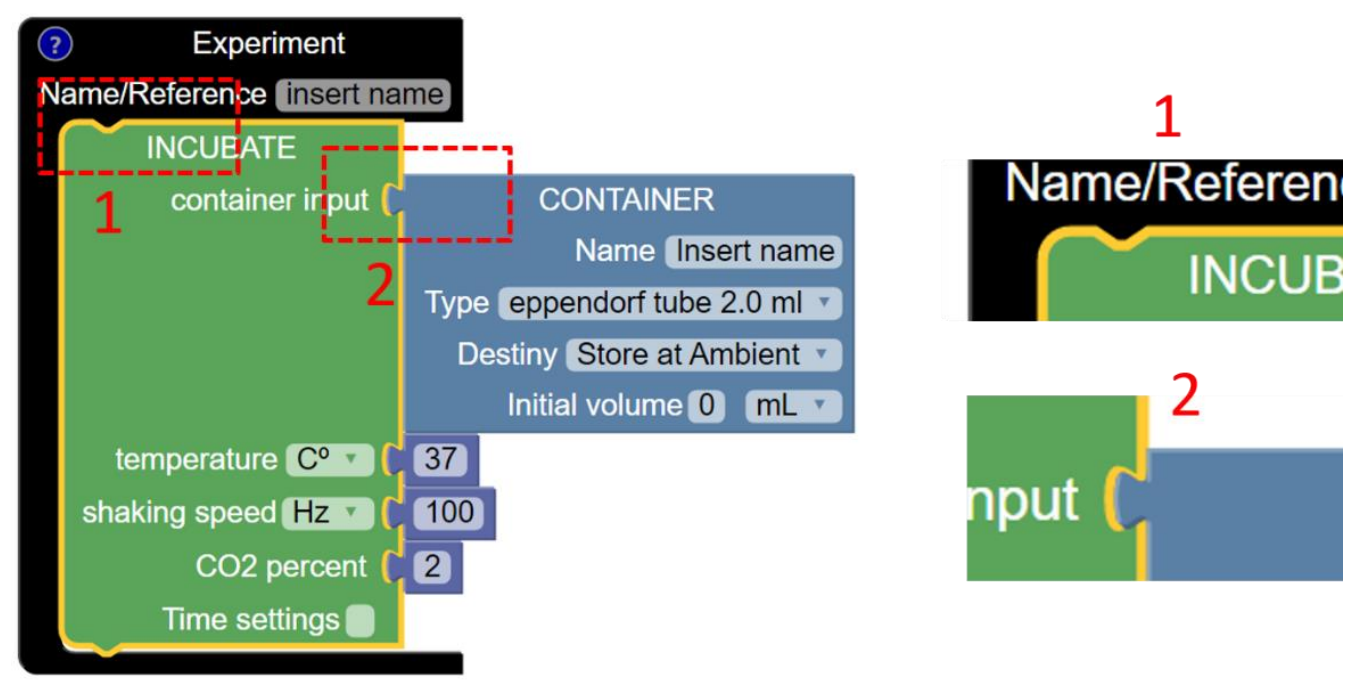

Figure 18 Visual Manipulation using connector types - There are two types of jig-saw like connectors available in Blockly. Connector 1 has been used to attach operation blocks, organization blocks and conditional blocks. Connector 2 is used for attaching container blocks and inputs to operation blocks. 

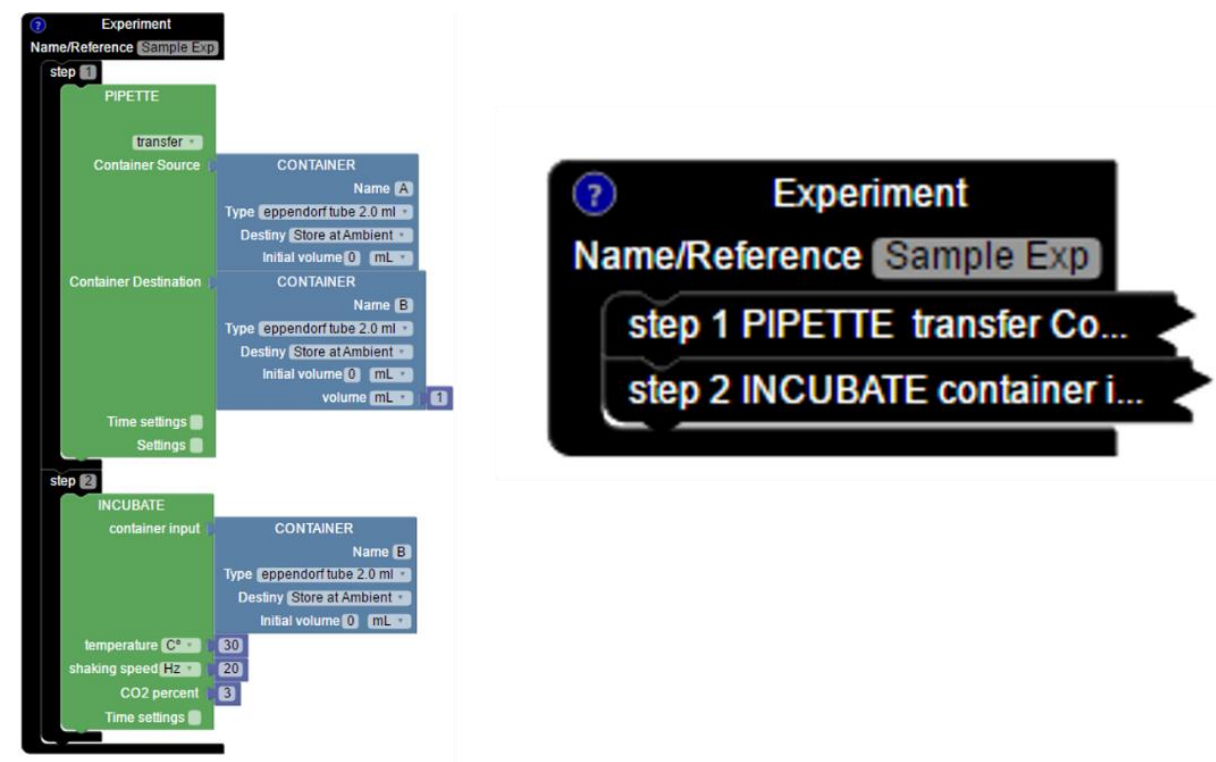

Figure 19 Visual Manipulation using Expanding/Collapsing Blocks - BioBlocks use a very powerful Blockly feature of Collapsing and Expanding Blocks to allow for improved visual manipulation of specified protocols. The protocol on the left can be collapsed for easier visual manipulation. The collapsed blocks on the right can be expanded back (restored) to its previous state.
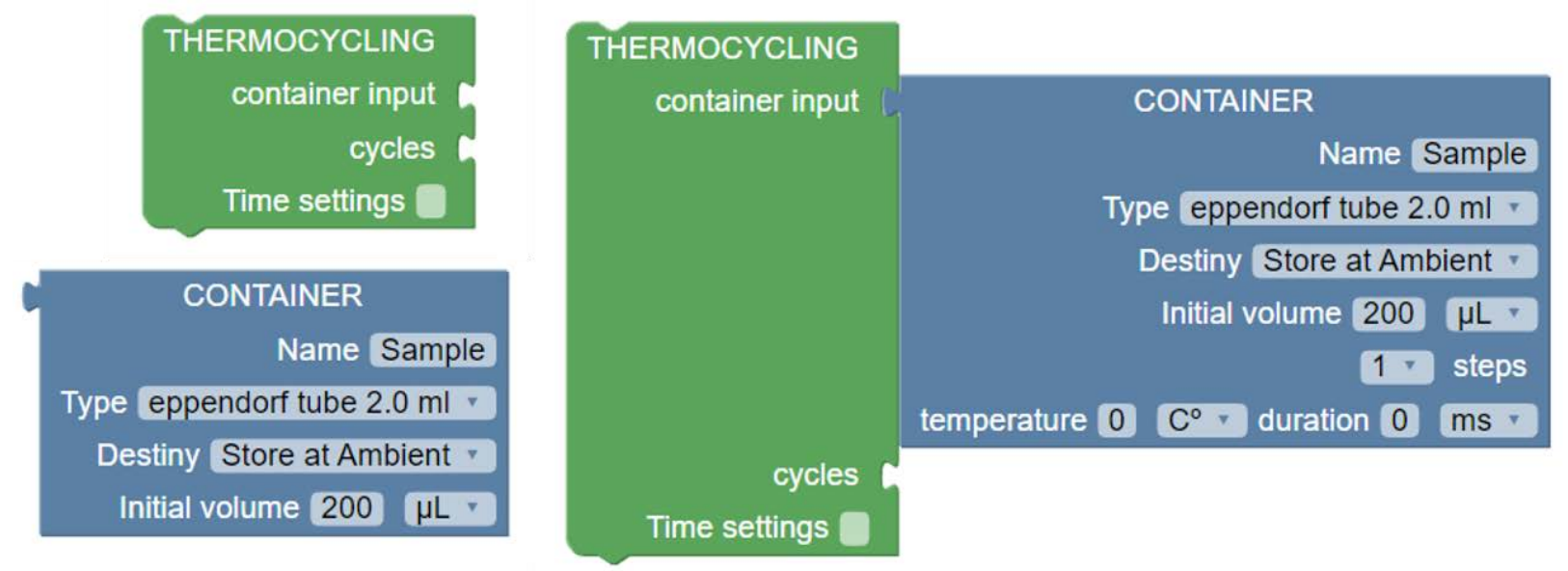

Figure 20 Dynamic Menu for progressive disclosure of inputs - The container block on the left (blue) displays more options depending on the operation block it has been attached to. In the above example, the container blocks on the right (blue) displays the number of steps it needs to thermocycle when used with a Thermocycling Operation Block. 


\subsubsection{Machine Specification App}

The Machine Specification App allows users to specify the milifluidic hardware on which the biological assays will be carried out. The specification can be carried out using blocks just like the Protocol Specification App. Currently, the most common way of representing hardware is in textual format is using netlists. The progress in hardware specification and automation has been largely inspired by the Electronic Design Automation (EDA) Industry. There are multiple solutions (Brisk, Aqua, CIDAR, Paul Pop) which have used the EDA paradigm of abstraction based on the logic, functionality, placement, mapping, routing and control for automating biological assays on different scales.

\section{Machine specification language}

A machine specification language was developed to allow users (specifically hardware engineers, DIY hardware enthusiasts) to define their milifluidic setups so that it can be used in an easy and convenient manner by biologists who have limited knowledge of designing, fabricating, assembling and executing experiments using microcontrollers.

The specification uses a 'part-machine' paradigm. A part can be any functional unit of machine. A part can be passive or active. Passive parts do not require actuation, for example a simple container with no additional functionality like mixing, heating. An active part can be actuated and the actuation behavior and control is specified separately in a file called the plugin file. The plugin file provides information to the BioBlocks framework for controlling the active part. Example of active parts are valves, pumps, containers with added functions like heating and measurement. Multiple parts together form a machine.

The machine specification language is designed keeping in mind future multi-platform compatibility i.e. milifluidic setups, microfluidic setups (continuous flow microfluidic chips, electrowetting on dielectric (EWOD) platforms) and pipetting robots (example TECAN, OpenTrons) can be specified using the specification language. For a milifluidic device, a part can be a container (e.g. tube, beaker), a pump or a valve. For a microfluidic device, a part can be an inlet, outlet, mixer, splitter, droplet generator etc. For an electrowetting on dielectric (EWOD) platform, a part can be an electrode which can contain a droplet. For a robotic platform, part is 
a well of microplate. Multiwell plates can be represented as multiple parts. This paradigm allows builders of setups to define not just their entire setups but individual parts. The partmachine paradigm has a very important advantage that is it makes the machines modular. Hence, allowing users to customize their machines by using different parts. Only milifluidic devices have been specified and tested within the scope of this work.

The Machine Specification Language consists of parts with a specific set of attributes. The attributes are Maximum Volume, Minimum Volume, Function/s (working range), State (open/close/bi) and Ports.

- The 'Maximum Volume' attribute allows users to define the maximum fluid capacity of a part. It can be an Eppendorf tube of $2 \mathrm{ml}$, a $150 \mathrm{ml}$ Scott-Duran beaker or pump which does not have any capacity to store fluids. In such cases the Maximum Volume can be defined as zero.

- The 'Minimum Volume' allow users to define the minimum working capacity of a part. This volume refers to the minimum volume that is required by the container to work adequately.

- The 'Function' attribute allows user to specify if the part has any functionality. In the case of active parts, like valves, pumps and other sensors, the functionality is defined as per the part behavior.

- The 'State' attribute defines if the part is an open container or closed. An open container in our specification requires actuation to drive the movement of fluid through it. A container in a closed state connected in series to a container in an open/closed state with actuation, does not require any further actuation to drive the fluid through it, as the pressure is maintained because of its closed state. The state attribute is very important as most machines (microfluidic and milifluidic) use closed containers. This is mainly due to the high cost of pumps. By maximizing the use of closed containers, the number of pumps needed to drive fluid flow is reduced. Hence, bringing down the cost of the setup. Also, the state attribute helps the compiler to help in control the flow of the fluid through the setup by turning ON/OFF the required actuators. 
- The 'Ports' attribute allows user to specify the number of connections in a part and the other its parts it relates to. The port attribute information is critical to build the machine layout.

\section{Graphical User Interface}

The block-based paradigm used in the Protocol Specification App is also used in the Machine Specification App to maintain a common graphical interface specification style. Therefore, a block-based graphical user interface (GUI) was designed and developed to define machines on which experiments can be executed. This allows users with little or no familiarity of using conventional textual specification like netlists, to specify their machines in a simple and convenient manner. The GUI is a part of the BioBlocks framework. It is a web-based tool and works in any popular web browser, e.g. Google Chrome. Users can describe their parts and upload the associated plugin files onto the local server. They can use these parts to specify a new machine or use an already pre-existing machine. The GUI is a drag and drop editor with constraints programmed into the way the blocks connect, to avoid errors arising from connecting incompatible parts.

\section{Specification of a Machine Layout}

The Machine Specification App consists of a library of parts and plugins. They can be used in a drag and drop fashion along with the organization block which is a single machine layout block (black) to specify setups. To specify a layout, users can first create the parts they will be needing from the part library. These parts currently can be open and closed container, pumps and valves. To these parts users can attach plugins to specify their functionality. The plugins are coded in python and can be safely assumed to be created by the hardware engineer who has designed and developed the part. Different parts have different plugin information. Container plugins have information about the Maximum and Minimum volume of the container. Pump and Valves plugins have information about behavior of their actuation and the number of ports. Users can additionally add any type of functions to a container by using the extra input on the 
part block. These functionalities can be heating, measurement of volume, temperature or OD (see Figure 21).
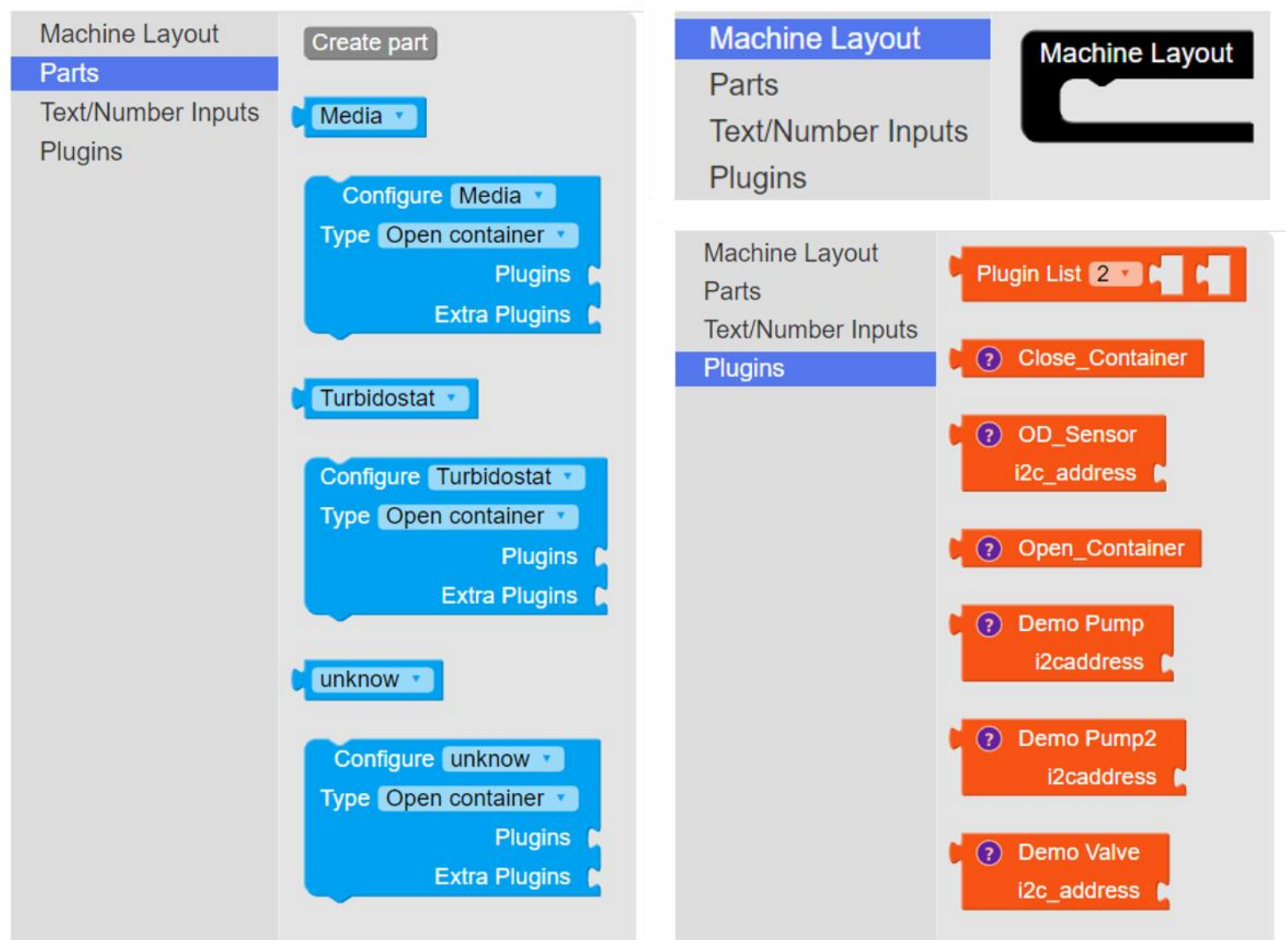

Figure 21 Creating a machine from the plugin library and parts - New parts (blue) can be created in the Machine Specification App (left). Pre-loaded plugins (orange) can be selected from the library (bottom right). The parts and plugins can be attached together along with machine layout block (black, top right).

\section{Machine Specification App outputs}

Just like the Protocol Specification App generates multiple outputs (English Translation, Protocol Workflow and Output code) to aid the user in cross validation of their specification and execution of experiment, the machine specification App also generate two outputs automatically in real-time. 
Machine Layout

The Machine layout output assists the users in validating if the hardware specification is correct. The layout is generated using the Cytoscape JS library, a very powerful data visualization tool. Cytoscape was used for generating the Protocol workflow as well in BioBlocks. The layout consists of different parts and the way they are connected depending on their specification. The parts are represented by nodes and the connection between them are represented by edges. To assist the user, the different parts with different functionality have been represented by different icons. A list of icons has been shown in the table below. The breadth-first algorithm from Cytoscape was used to generate the layout as it generates a clean layout for visualization of the machine.

Table 2 List of icons used to represent the machine layout in the Machine Specification App. The different icons represent different parts of a container.

\begin{tabular}{|l|l|}
\hline Name & Icons \\
\hline Open Container & \\
\hline Pump uni- directional / bi-directional
\end{tabular}




\section{Output Code}

The App also generates an output code which machine readable specification of the code. It is formatted in JSON and is required by the Protocol Execution App to understand the layout and its plugins to be able to execute an experiment on the machine (See Appendix for Output code).
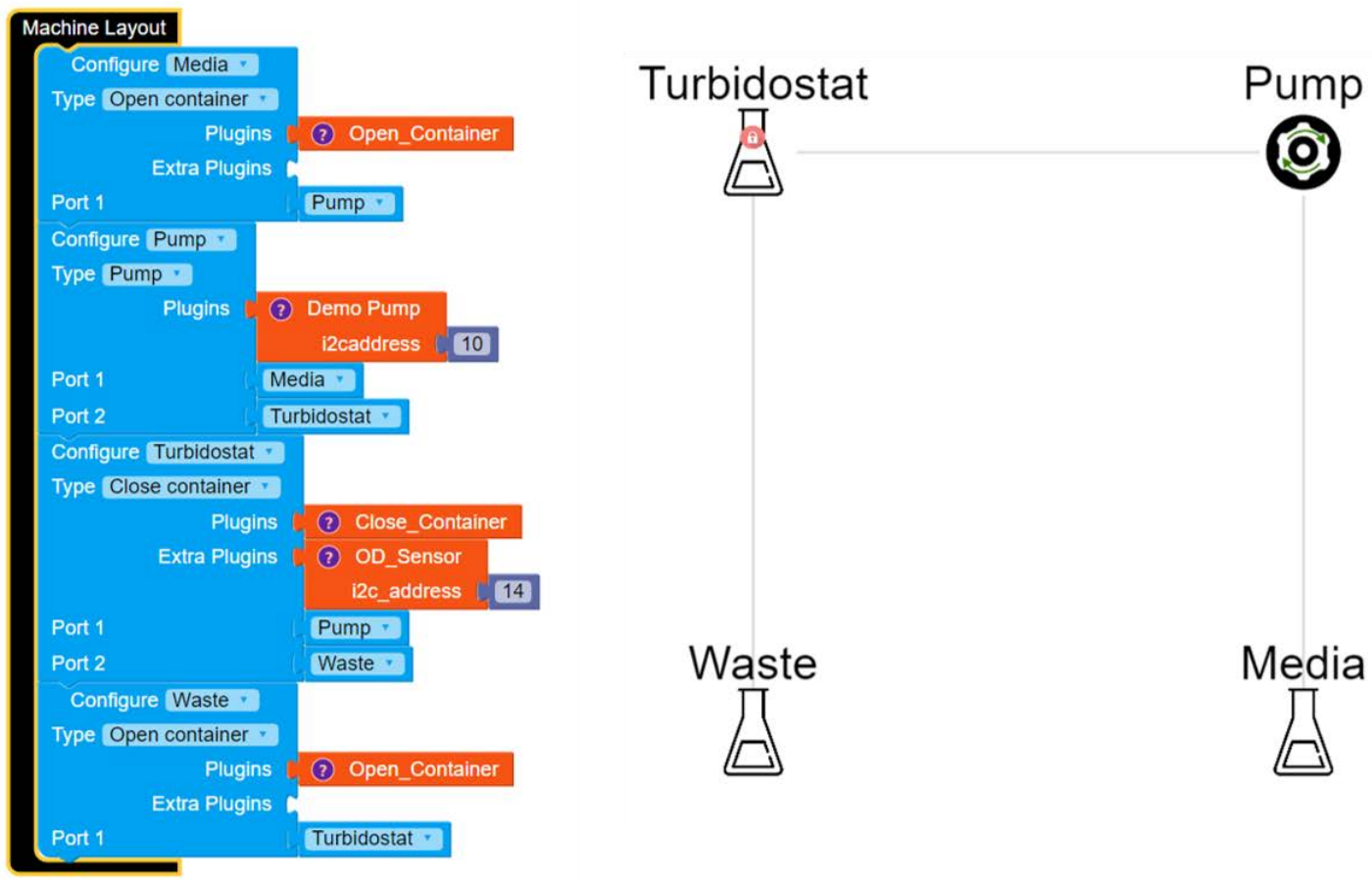

Figure 22 Machine Specification App example - A continuous flow millifluidic device called a Turbidostat was specified in the App. The block-based specification using parts (blue) and plugins (orange) is shown on the left. The automated real-time output of the machine layout is displayed on the right. 


\subsection{EXECUTION LEVEL}

To develop and end to end Automation solution the Protocol Execution App was developed. After the specification or selection of a pre-specified protocol and machine, the protocol needs to be executed on the machine. The execution of the protocol over a machine is a complex process which is done in multiple steps. The Execution App is a software tool which can take the protocol and machine specifications as inputs and sending the instructions in real-time to the microcontroller to execute a protocol. It produces the commands that can be understood by an Arduino board with the adequate firmware pre-loaded. The main objective of this section is to show the transformation and adaptation process required for the end-to-end solution. It closes the gap between the platform independent specification of the protocol using a GUI and its physical execution on a pre-specified machine.

The Protocol Execution App serves as the middleware between the specification and execution. Abstraction is the main concept that guides the different modules of the App. The App abstracts low level details of the devices and the microcontroller such as the control and behavior, from the user. This allows user to focus on high-level specification of the assay and its optimization to obtain the desired results.

\subsubsection{Protocol Execution App architecture}

The App has a very modular architecture. The modularity of the architecture has been a central theme in its design and development. It's different modules which can be replaced with other modules to make it compatible with different types of machines such as continuous flow microfluidic devices. Currently the Execution App only supports execution on millifluidic devices. 


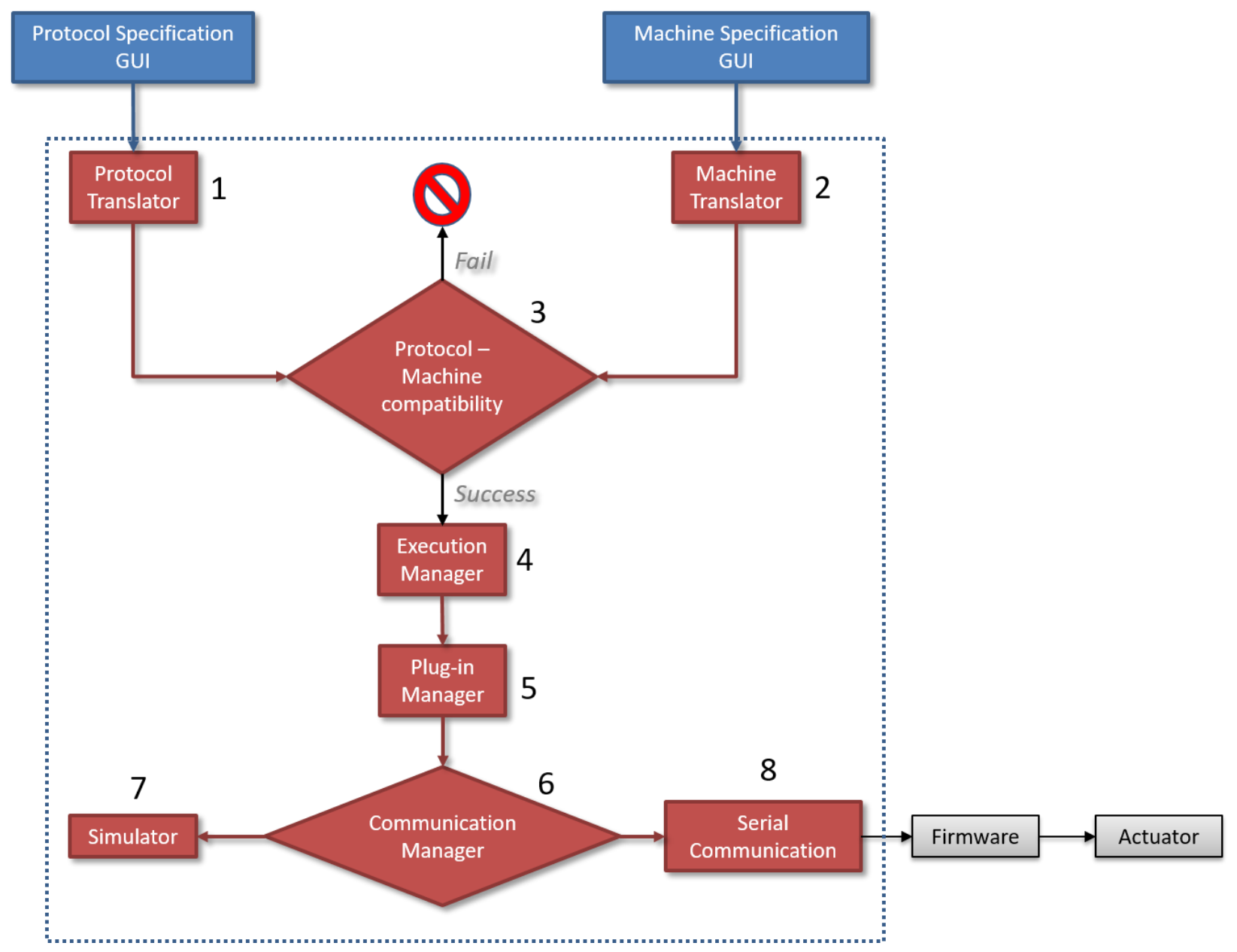

Figure 23 Architecture of the Protocol Execution App - The different modules present in the Protocol Execution App are shown in Maroon. The protocol and the machine specifications are first transformed into internal graphs (1,2), followed by a compatibility check (3) and finally instructions are generated after gathering device specific information from the plugin Manager $(4,5)$ which are sent to the micro-controller $(6,8)$. The user also has an option to generate a simulation of the protocol execution (7).

\section{Working of the Protocol Execution App}

The App takes the specification of the Protocol and the Machine specified in the BioBlocks Automation Framework as inputs. It then carries out a series of transformation, checks and scheduling to allow for automated execution of a milifluidic protocol. The working of the Protocol Execution App is mentioned below- 
Step 1 - A protocol specified in BioBlocks needs to be first translated into compiler compatible JSON. It is then transformed into a graph model of the protocol.

Step 2 - The machine specification is also simultaneously transformed into graph model by the compiler.

Step 3 - The compiler then performs a compatibility check to see if the protocol can be executed on the machine.

Step 4 - If the protocol and the machine are a compatible, a compatibility table is generated.

Step 5 - Instructions are generated to be sent to the microcontroller for executing the experiment.

Protocol Execution App functionalities

Protocol Transformation from JSON to EvoCoder

The protocol specified in BioBlocks framework is automatically translated to JSON format. It is a modified form of Autoprotocol language. The protocol is transformed into the internal representation because it allows us to perform operations over it. A modified version of EvoCoder is used for the transformation. To see the entire list of EvoCoder primitives see Appendix. Time is a complex parameter to handle when the protocols specified in BioBlocks are transformed to a graph model for execution. At the specification level the user can specify time in a manner which is familiar to the user. However, for execution purposes time parameter is handled in a different manner. The time parameter is discretized into time slices along with a 'time step' parameter. The user can decide the time step parameter or alternatively choose between a low, medium or high value when executing the protocol.

In the internal graph model, the nodes are operations and the edges are conditions that must be met to proceed to the next operation (See Figure 24). To execute a protocol, we always start at a node (tagged as start node) and checks are performed to calculate which leaving edges meet the conditions to be executed. The Graphs are Directed graphs. If the condition of the 
edge is met, the next pointing node is executed. When there are no more edges leaving a node where the conditions can be met, the execution of the protocol is halted.

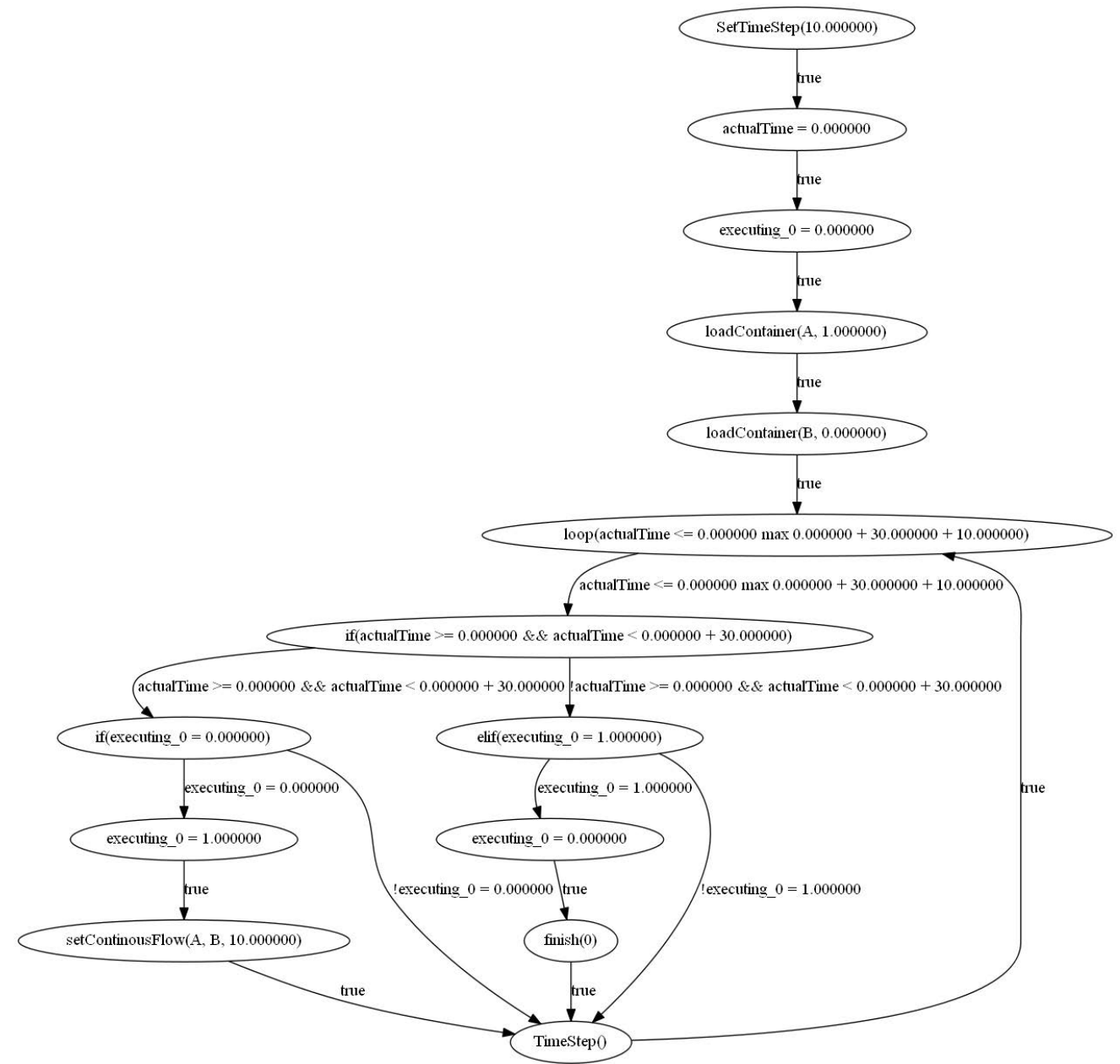

Figure 24 Graph Model of the protocol - The protocol is transformed to a graph model for internal representation so that operations can be performed over it. The nodes are operations and the edges are conditions.

\section{Protocol Mapping}

Biologists are continuously prototyping different types of experiments when doing their research. During prototyping the requirements of a protocol to be executed on a machine can vary. It is critical to ensure that a protocol specified by a user is compatible with the machine on which it needs to be executed. Also, protocols specified in the Protocol Specification App are independent of the platform. Therefore, a protocol-machine compatibility check is compulsory. 
To perform a protocol-machine compatibility check the protocol is mapped to the required machine. In mapping, the protocol-machine compatibility module looks for a set of containers in the machine that can be used to execute a protocol. Mapping is performed as follows -

- First, the requirements are extracted from a protocol. The containers along with their attributes like their volume, open/closed state, operations or functions that need to be performed over the container are gathered. The liquid movements (discrete or continuous flow) are also resolved and maintained in time. This is to check whether a protocol (which are specified in a platform agnostic manner) can be executed on a machine.

- Then a set of containers which meet the requirements for execution from the protocol are searched on the specified machine. This search is performed using $A^{*}$ algorithm. The approach used is to find a working solution and not be concerned with the quality/fitness of the solution. $A^{*}$ space search algorithm is used because it ensures that if a solution exists it is eventually obtained.

- If a solution is found, the mapping is a success. A solution is a subset of machine containers that can be used to execute the protocol. If no solutions are found, the mapping fails and the protocol is not compatible with the machine.

\section{Execution}

Protocols specified in BioBlocks Framework are platform independent to allow eventual execution on different platforms based on the requirement of the user. The Execution manager receives instructions for compatible containers on the machine. There are two types of operations which can be performed, 1) Which require a series of containers and 2) which require only individual containers. The former operation is centered on the fluidic paradigm and requires routing. Whenever routing is required the Execution manager communicates with the plugin manager to communicate with the containers. This is followed by routing. As explained earlier, the execution manager uses the internal graph model for execution of the protocol (See Figure 24). 


\section{Routing}

In a machine setup, there are several ways to maintain a flow between two containers. To set a flow between two containers we need to find path between them. Along with the path we also need information regarding the states of the pumps and valves to route the liquid flow by setting the appropriate states for the actuators.

Routing is performed by transforming the machine specifications into a set of constraints. Constraints are equations which need to be satisfied to obtain a successful routing. The equations are fed values which are obtained from the protocol specifications. If the values are optimal which means that protocol requirements of the liquid flow are possible, the routing engine returns the state of all the parts (containers, pumps etc.). Once the state of all the parts are obtained the communication manager communicates with microcontroller to send instructions to execute the protocol.

\subsubsection{Examples}

The BioBlocks Automation framework's end to end automation capability can be demonstrated with the help of the following example. Turbidostat is a continuous flow fluidic device where the cell density of a cell culture is maintained. It consists of three containers: Media Container, Turbidostat Container and Waste Container. It also has a pump which pumps liquid between Media-Turbidostat-Waste Container and a sensor to measure Optical Density (OD).

Turbidostat protocol in English -

- Transfer $50 \mathrm{ml} / \mathrm{hr}$ of liquid from Media Container to Cell Culture Container for 10 hours.

- Transfer $50 \mathrm{ml} / \mathrm{hr}$ of liquid from Cell Culture Container to Waste container for 10 hours.

- Measure the OD of the Cell Culture container. (20 Hz frequency, $600 \mathrm{~nm}$ wavelength) for 10 hours.

- keep the OD constant around value of 1 by changing the flow rate between the Media, Cell Culture and Waste containers to Turbidostat

Protocol in BioBlocks - 


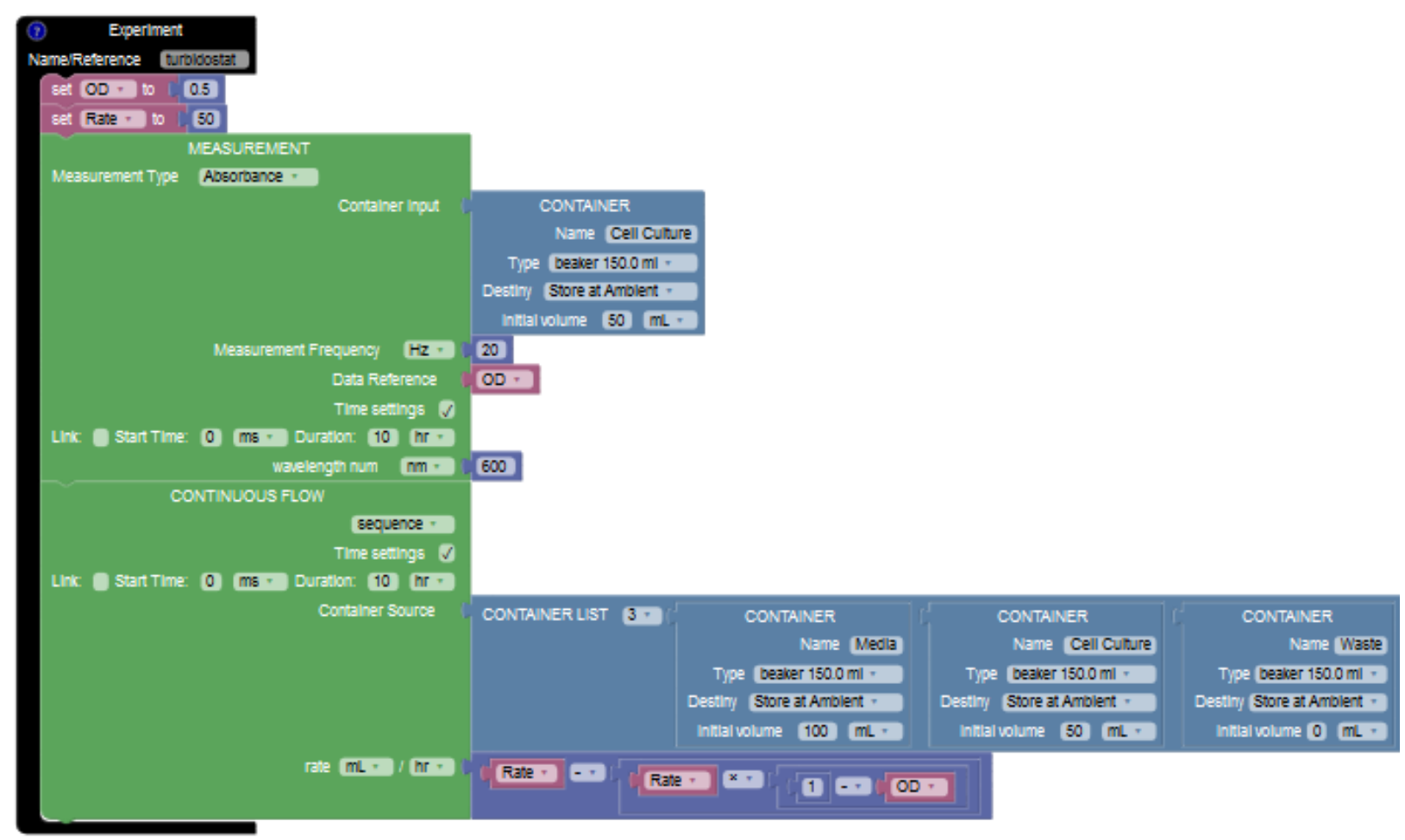

Figure 25 Turbidostat Protocol in BioBlocks in the BioBlocks framework -the Continuous flow operation block (green) a Turbidostat can be easily specified. In the protocol, the Rate has been set proportional to the change in OD value.

27 17:43:14 . setting time step to 1

27 17:43:14 . fill machine's container: 1 with $50.000000 \mathrm{ml}$ of protocol's container: Cell Culture, click OK when ready.

27 17:43:16 . fill machine's container: 0 with $100.000000 \mathrm{ml}$ of protocol's container: Media, click OK when ready.

27 17:43:17 . fill machine's container: 3 with $0.000000 \mathrm{ml}$ of protocol's container: Waste, click OK when ready.

27 17:43:19 . start measuring OD from Cell Culture with: measurement frequency: 20 and waveLength: $6 \mathrm{e}-07$

27 17:43:19. set continuous flow from Media to Cell Culture at 6.94444e-06

27 17:43:19. set continuous flow from Cell Culture to Waste at 6.94444e-06

\section{[...] 10 hours}

27 03:43:49 . stop measuring OD of Cell Culture.

27 03:43:49 . stop continuous flow from Media to Cell Culture

27 03:43:49 . stop continuous flow from Cell Culture to Waste 
Figure 26 The Execution of the Turbidostat using the Protocol Execution App - The Turbidostat Protocol (Figure 25) and the Turbidostat setup (Figure 22) were uploaded in the Protocol Execution App. The resultant generated instructions generated as displayed in the console is shown above. The App prompts user to click OK after loading the respective containers. The output has been condensed for brevity. 


\subsection{DISCUSSION OF THE FRAMEWORK}

There are several important aspects of the of the automation framework that need to be highlighted along with their advantages and limitations.

\section{Platform Independent Specification}

The Platform Specification App has been designed to capture experimental protocol specification, in a platform independent manner. There are various platforms available today like pipetting robots, microfluidic and milifluidic devices on which experimental protocols can be executed. Moreover, each platform has multiple commercial and academic products with their own unique set of instructions to execute an experimental protocol. To ensure a useful solution, the specification of protocols was abstracted from the execution platform. The translation/transformation of the protocol to a specific platform is handled by the Execution App. Since, the specification is platform independent the user needs to add the machine specification himself and can currently automate the protocols only on fluidic devices.

\section{Protocol Specification as Top layer}

BioBlocks at the specification level has been carefully designed to serve as top layer to other automation tools. Its architecture has been developed keeping in mind interoperability with multiple software tools on the same specification level or below it (execution level). Interoperability is the ability to connect with external automation solutions, was a primary objective of this work. We wanted to build a solution which could connect with external solutions like Transcriptics, Antha (bio-languages) OpenTrons, Tecan (Robots) to improve its impact. (See Figure 27)

Since BioBlocks is open-source, the output of the BioBlocks can be modified to translate a protocol in blocks to be compatible with another software tool. The first version of BioBlocks was designed to output code in Autoprotocol language. The current version of BioBlocks output code is a modified version of Autoprotocol. The previous and newer versions are available on the GitHub (https://github.com/liaupm). However, it is important to keep in mind that the open-source nature of the Framework allows people to maintain their own customized 
framework. This could potentially create multiple frameworks which would not be compatible and hence it would be difficult to share protocol and machine specifications.

Modularity

The Machine Specification and the Protocol Execution Apps have been designed to be very modular so that the community can extend the work to allow end to end automation of protocols on multiple hardware platforms. Currently, the Machine Specification App and Protocol Execution App only support specification and execution over millifluidic devices. They can be extended with minor alterations to define most setups. Also, the alterations are mostly focused in the plugin files as the setups differ mainly in their method of actuation. The design allows specification of other platforms with a small effort. However, to extend support to other platforms at the level of execution would require software development expertise along with working knowledge of the platform. 


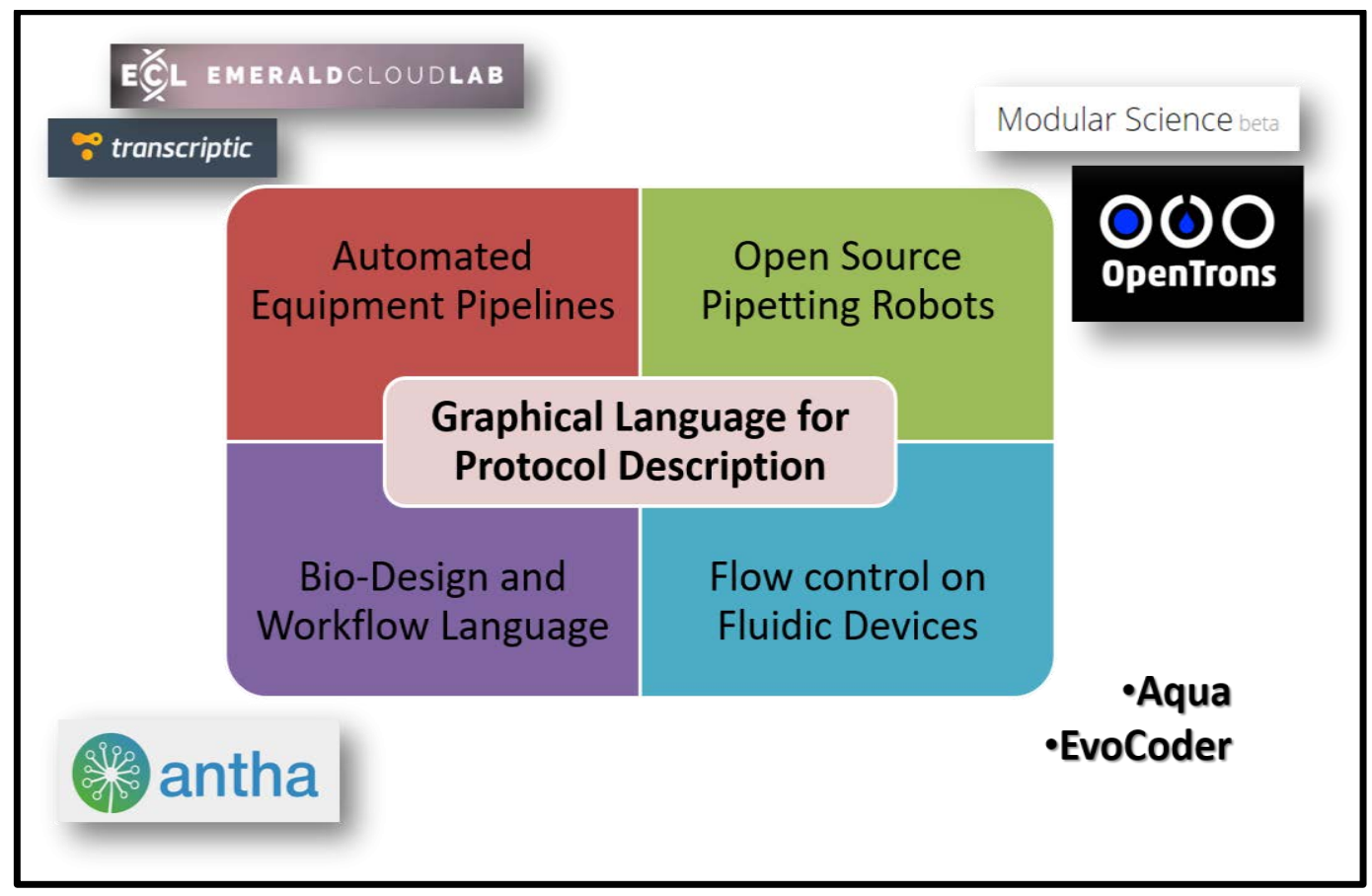

Figure 27 Aimed Interoperability of BioBlocks - BioBlocks was designed to be able to connect with multiple solutions like Automated Equipment Pipelines likes Transcriptics, Pipetting Robots like OpenTrons, flow control devices like Aqua and EvoCoder and Bio-design and workflow languages like Antha.

Platform-based Architecture

The BAF has been designed as platform which facilitates exchange between biologist and hardware engineers. The hardware engineers can design and build parts (or entire machines) along with their plugins and add it to the BAF for biologists to program their machines. They can just build parts and are not forced to build entire machines, since parts can be specified and shared. This makes it easier for both the users as the biologists do not need to design and build parts and the hardware engineers do not need to build a software interface for their devices. A platform would be successful if it was implement on the web. However, the maintenance of the platform and curation of working parts requires money, time and effort. 
Validation

BAF is designed to display multiple outputs on both the specification levels, that is protocol and machine. The protocol specifications outputs English Translation of the protocol, Protocol workflow and machine-readable code. The machine specification outputs Machine layout and machine-readable code. The English translation, the protocol workflow and the machine layout help the users to validate the specified design. Even though a block-based design allows users a programming-free specification of their designs. It is easier to share and review protocols with English Translation and workflows/layouts. Cytoscape is used for generating the graphs is a feature rich graph library. It generates interactive dynamic graphs where nodes and edges can be moved and the workflow/layout can be panned and zoomed in/out. 


\section{CONCLUSIONS AND FUTURE WORK}

\subsection{CONCLUSIONS}

Two research questions were posed at the beginning of the work:

Research question 1 explored the possibility of developing a high-level language for specifying platform-agnostic protocols in experimental biology which required no programming knowledge. The Protocol Specification Application in the BioBlocks Automation Framework (BAF) allows users to specify their protocols using a block-based graphical interface inspired by MIT's Scratch and Google's Blockly. The user requires little or no programming knowledge and the protocols specified are platform agnostic.

The Protocol Specification App was published as BioBlocks in the pre-print server bioRxiv and eventually in ACS Synthetic Biology. It was very well received by the scientific community and industry alike. Many companies including Transcriptic, Lattice Automation and OpenTrons approached us expressing their interest in using BioBlocks as a potential tool for specification. Apart from that we have also won the first round of the Universidad Politecnica de Madrid (UPM) annual Business competition called ActuaUPM. BioBlocks has immense potential to be the de facto standard for specification of protocols in experimental biology. However, an active community is needed to define the scope of the types of operations and number of standard operations which should be available in the BioBlocks library to cover specification of maximum protocols.

Research Question 2, explored the possibility of developing an end-to end solution which allows the execution of a platform-agnostic specification of a protocol, on a compatible hardware platform of the user's choice. This led to development of the Machine Specification App and Protocol Execution App. Machine Specification App allows users to specify their machines on which the protocol will be executed. It too uses a block-based graphical interface to reduce the programming burden while specification. The Protocol Execution App takes care of the execution part. Once, the users have specified their protocol and machine in the framework, 
this App automates the execution of the protocol on the machine. Hence, achieving the endto-end automation solution.

The BioBlocks automation framework is open source and has been developed to serve as a platform to connect Biologist with Hardware Engineers, Do-it-Yourself (DIY) enthusiasts. Biologists have the advantage that they do not need to learn the low-level details of the machines. On the other hand, Hardware engineers and DIY enthusiasts do not need to design a front-end interface for biologists to use their machines or parts. With BAF we have attempted to lower the automation barrier so that users can have access to automation and highthroughput analysis. With the advent of Synthetic Biology such a framework would be very useful for scientists for rapid-prototyping of their experiments. 


\subsection{FUTURE WORK}

The BioBlocks Automation Framework (BAF) has demonstrated both academic and commercial potential. There are many possibilities which can be explored with BAF. Some possible future directions are:

On the academic front-

- Currently protocols in BAF are being specified by users manually. In the future, it would be very useful to generate blocks automatically from English using Natural Language processing. A user should be able to upload a protocol of his choice in English or other languages and BAF should automatically parse the protocol into BioBlocks.

- It would be useful to extend the Machine Specification App and Protocol Execution App to support to continuous flow microfluidic devices and Electrowetting on Dielectric (EWOD) microfluidic platforms. This would allow BAF users access to high-throughput studies at lower costs.

- BAF currently has only a few parts and machines defined. It would be ideal for BAF to collaborate with different communities like Thingiverse ${ }^{68}$ and Metafluidics ${ }^{69}$ so that users can upload the machine specifications (layout not physical design) and plugins to allow programmability and control of the machines.

- It would be interesting to add capabilities to specify not just protocols but also genetic circuits. LIA is currently developing a block-based prototype for specifying complex genetic circuits.

- BAF allows specification of complex conditional protocols like Turbidostats and Chemostats. Sometimes the protocols can be visually daunting to modify as there are many parameters that can be tuned. However, in practice only a couple of parameters are optimized for a given experiment. Work is being done is this direction to create Metablocks, which allows the user to shrink experiments in a customizable manner. This would allow a user to only display parameters that need to be optimized thereby improving the usability of the interface. 
- To establish BAF as the standard for sharing protocol specifications by publishing methods in paper using BioBlocks. The whole experiment can be shared as an executable which can be automated in local or cloud laboratories to improve the reproducibility of science.

On the commercial front-

- We will participate in the second round of UPM Business competition called ActuaUPM. We are currently exploring different business plans to build BAF into a sustainable business. One business plan idea is to start a technology based consultancy which assists automation companies to customize BioBlocks to their products.

- We are also applying for the EU FET LaunchPad grant. The grant allots money specifically for commercialization of research outputs generated from other FET calls. 


\section{REFERENCES}

1. Begley, G. \& Ellis, L. Drug development: Raise standards for preclinical cancer research. Nature 483, 531-533 (2012).

2. Leek, J. \& Peng, R. Opinion: Reproducible research can still be wrong: Adopting a prevention approach. Proceedings of the National Academy of Sciences 112, 1645-1646 (2015).

3. Ioannidis, J. How to Make More Published Research True. PLoS Medicine 11, (2014).

4. Balaban, N., Gerdes, K., Lewis, K. \& McKinney, J. A problem of persistence: still more questions than answers? Nature reviews. Microbiology 11, 587-91 (2013).

5. Lowndes, J. et al. Our path to better science in less time using open data science tools. Nature Ecology \& Evolution 1, 0160 (2017).

6. Freedman, L. \& Inglese, J. The increasing urgency for standards in basic biologic research. Cancer research 74, 4024-9 (2014).

7. Bilofsky, H. S. \& Burks, C. The GenBank genetic sequence data bank. Nucleic Acids Res. 16, 1861-3 (1988).

8. Pearson, W. R. \& Lipman, D. J. Improved tools for biological sequence comparison. Proc. Natl. Acad. Sci. U.S.A. 85, 2444-8 (1988).

9. Galdzicki, M. et al. The Synthetic Biology Open Language (SBOL) provides a community standard for communicating designs in synthetic biology. Nature Biotechnology 32, 545-550 (2014).

10. Roehner, N. et al. Proposed Data Model for the Next Version of the Synthetic Biology Open Language. ACS Synthetic Biology 4, 57-71 (2015).

11. Galdzicki, M., Rodriguez, C., Chandran, D., Sauro, H. \& Gennari, J. Standard Biological Parts Knowledgebase. PLoS ONE 6, (2011).

12. De Lorenzo, V. \& Schmidt, M. Biological standards for the Knowledge-Based BioEconomy: what is at stake. New Biotechnology (2017). doi:10.1016/j.nbt.2017.05.001

13. Konopka, B. Biomedical ontologies - A review. Biocybernetics and Biomedical Engineering 35, 75-86 (2015).

14. Soldatova, L., Aubrey, W., King, R. \& Clare, A. The EXACT description of biomedical protocols. Method Biochem Anal 24, i295-i303 (2008). 
15. Giraldo, O, García, A \& Corcho, O. SMART protocols: semantic representation for experimental protocols. ... of the 4th International Conference on ... (2014). at $<$ http://dl.acm.org/citation.cfm?id=2878588>

16. Beal, J., Phillips, A., Densmore, D. \& Cai, Y. High-level programming languages for biomolecular systems. Springer 225-252 (2011). doi:10.1007/978-1-4419-6766-4_11

17. Bilitchenko, L., Liu, A. \& Densmore, D. The Eugene language for synthetic biology. Methods in enzymology 498, 153-72 (2011).

18. Beal, J \& Bachrach, J. Cells are plausible targets for high-level spatial languages. SelfAdaptive and Self-Organizing Systems ... (2008). at $<$ http://ieeexplore.ieee.org/abstract/document/4800692/>

19. Villalobos, A \& Ness, JE. Gene Designer: a synthetic biology tool for constructing artificial DNA segments. $B M C$... (2006). at $<$ https://bmcbioinformatics.biomedcentral.com/articles/10.1186/1471-2105-7-285>

20. Benchling. Benchling.

21. -. Genome Compiler.

22. Xia, B. et al. Developer's and user's guide to Clotho v2.0 A software platform for the creation of synthetic biological systems. Methods in enzymology 498, 97-135 (2011).

23. Bhatia, S. \& Densmore, D. Pigeon: a design visualizer for synthetic biology. ACS synthetic biology 2, 348-50 (2013).

24. CIDAR Lab. Merlin.

25. Wang, H. et al. Programming cells by multiplex genome engineering and accelerated evolution. Nature 460, 894-898 (2009).

26. -. DNA 2.0.

27. -. Integrated DNA Technologies.

28. -. OpenTrons.

29. -. Modular Science.

30. -. Open PCR.

31. -. Kilo Baser.

32. -. Ark Reactor.

33. Matteau, D., Baby, V., Pelletier, S. \& Rodrigue, S. A Small-Volume, Low-Cost, and Versatile Continuous Culture Device. PLOS ONE 10, e0133384 (2015). 
34. Callens, C. et al. A multiplex culture system for the long-term growth of fission yeast cells. Yeast (2017). doi:10.1002/yea.3237

35. Nadal, D., Ammerer \& Posas. Controlling gene expression in response to stress. (2011). doi: $10.1038 / \operatorname{nrg} 3055$

36. Takahashi, C., Miller, A., Ekness, F., Dunham, M. \& Klavins, E. A Low Cost, Customizable Turbidostat for Use in Synthetic Circuit Characterization. ACS Synthetic Biology 4, 32-38 (2015).

37. Miller, A. W., Befort, C., Kerr, E. O. \& Dunham, M. J. Design and use of multiplexed chemostat arrays. J Vis Exp e50262 (2013). doi:10.3791/50262

38. Toprak, E. et al. Building a morbidostat: an automated continuous-culture device for studying bacterial drug resistance under dynamically sustained drug inhibition. Nat Protoc 8, 555-67 (2013).

39. -. Evolvinator.

40. Shih, S. C. C. et al. A Versatile Microfluidic Device for Automating Synthetic Biology. ACS Synth Biol 4, 1151-64 (2015).

41. Kerhoas, K. et al. Validation of a fully integrated platform and disposable microfluidic chips enabling parallel purification of genome segments for assembly. Biotechnology and bioengineering 111, 1627-1637 (2014).

42. Yehezkel, T. et al. Synthesis and cell-free cloning of DNA libraries using programmable microfluidics. Nucleic Acids Research 44, e35-e35 (2016).

43. Gach, P. et al. A Droplet Microfluidic Platform for Automating Genetic Engineering. ACS Synthetic Biology (2016). doi:10.1021/acssynbio.6b00011

44. Woodruff \& Maerkl. A High-Throughput Microfluidic Platform for Mammalian Cell Transfection and Culturing. (2016).

45. Linshiz, G. et al. End-to-end automated microfluidic platform for synthetic biology: from design to functional analysis. Journal of Biological Engineering 10, 3 (2016).

46. -. Autoprotocol.

47. -. Emerald Cloud Lab.

48. -. Transcriptic.

49. -. Antha.

50. -. Synthace. 
51. -. Gingko Bioworks.

52. -. Zymergen.

53. -. Project Cyborg.

54. Thies, W., Urbanski, J., Thorsen, T. \& Amarasinghe, S. Abstraction layers for scalable microfluidic biocomputing. Natural Computing 7, 255-275 (2007).

55. Ananthanarayanan, V. \& Thies, W. Biocoder: A programming language for standardizing and automating biology protocols. Journal of Biological Engineering 4, 1-13 (2010).

56. Linshiz, G. et al. PaR-PaR Laboratory Automation Platform. ACS Synthetic Biology 2, 216-222 (2013).

57. Vasilev, Liu, Haddock \& Bhatia.... A software stack for specification and robotic execution of protocols for synthetic biological engineering. (2011).

58. Huang, H. \& Densmore, D. Fluigi: Microfluidic Device Synthesis for Synthetic Biology. ACM Journal on Emerging Technologies in Computing Systems (JETC) 11, 26 (2014).

59. -. Neptune.

60. -. CIDAR Lab Microfluidics.

61. McDaniel, Baez, Crites, Tammewar \& Brisk. Design and verification tools for continuous fluid flow-based microfluidic devices. 219-224 (2013).

doi:10.1109/ASPDAC.2013.6509599

62. McDaniel, J., Curtis, C. \& Brisk, P. Automatic synthesis of microfluidic large scale integration chips from a domain-specific language. 101-104 (2013).

doi:10.1109/BioCAS.2013.6679649

63. Amin, A., Thottethodi, M., Vijaykumar, Wereley, S. \& Jacobson, S. AquaCore: A programmable architecture for microfluidics. 35, 254-265 (2007).

64. Amin, A. et al. Software-programmable continuous-flow multi-purpose lab-on-a-chip. Microfluidics and Nanofluidics (2013). doi:10.1007/s10404-013-1180-2

65. -. Blockly.

66. Gupta, V., Irimia, J., Pau, I. \& Rodriguez-Paton, A. BioBlocks : Programming protocols in biology made easier. ACS Synthetic Biology (2017). doi:10.1021/acssynbio.6b00304

67. Shannon, P. et al. Cytoscape: a software environment for integrated models of biomolecular interaction networks. Genome research 13, 2498-2504 (2003).

68. -. Thingiverse. 
69. Kong, D. et al. Open-source, community-driven microfluidics with Metafluidics. Nature Biotechnology 35, 523-529 (2017). 


\section{ANNEXI}

Autoprotocol a formal language to specify experimental protocols was used to develop the initial library of Operations in BioBlocks. However, the instructions available in Autoprotocol was limited and more instructions were added in to improve its capability to specify more complex experiments like continuous flow experiments. The list of Autoprotocol instructions represented in BioBlocks and the missing ones are shown below.

Table 3 List of BioBlocks and their respective Autoprotocol instructions

\begin{tabular}{|l|l|}
\hline BioBlocks & Autoprotocol Instruction \\
\hline Container Block & Container Access \\
\hline Pipette Block & $\begin{array}{l}\text { One-Channel Liquid Handling } \\
\text { Multichannel Liquid Handling } \\
\text { Acoustic Liquid Handling } \\
\text { Reagent Dispensing }\end{array}$ \\
\hline Colony Picking Block & Colony Picking \\
\hline Cell spreading Block & Cell Spreading \\
\hline Sanger Sequencing Block & Sanger Sequencing \\
\hline Thermocycling Block & Thermocycling \\
\hline Centrifugation Block & Centrifugation \\
\hline Incubate Block & Incubation \\
\hline Electrophoresis Block & Gel Electrophoresis \\
\hline Measurement Block & $\begin{array}{l}\text { Measure Property } \\
\text { Spectrophotometry }\end{array}$ \\
\hline Oligosynthesize Block & Oligosynthesize \\
\hline Flash Freeze Block & Flash Freeze \\
\hline Flow Cytometry Block & Flow Cytometry \\
\hline Not available & Magnetic Separation \\
\hline Mix Block & Not available \\
\hline & 83 \\
\hline
\end{tabular}


Continuous Flow Block

Not available

Not available

Covers and Seals 


\section{ANNEX II}

EvoCoder is a high-level language for protocol specification. The language developed abstracted all the low-level hardware and electronic details. It was based on $\mathrm{C}++$ and consisted of a simple library (collection) of primitives (commands) that are based on fluid manipulation like move, mix etc. (See below).

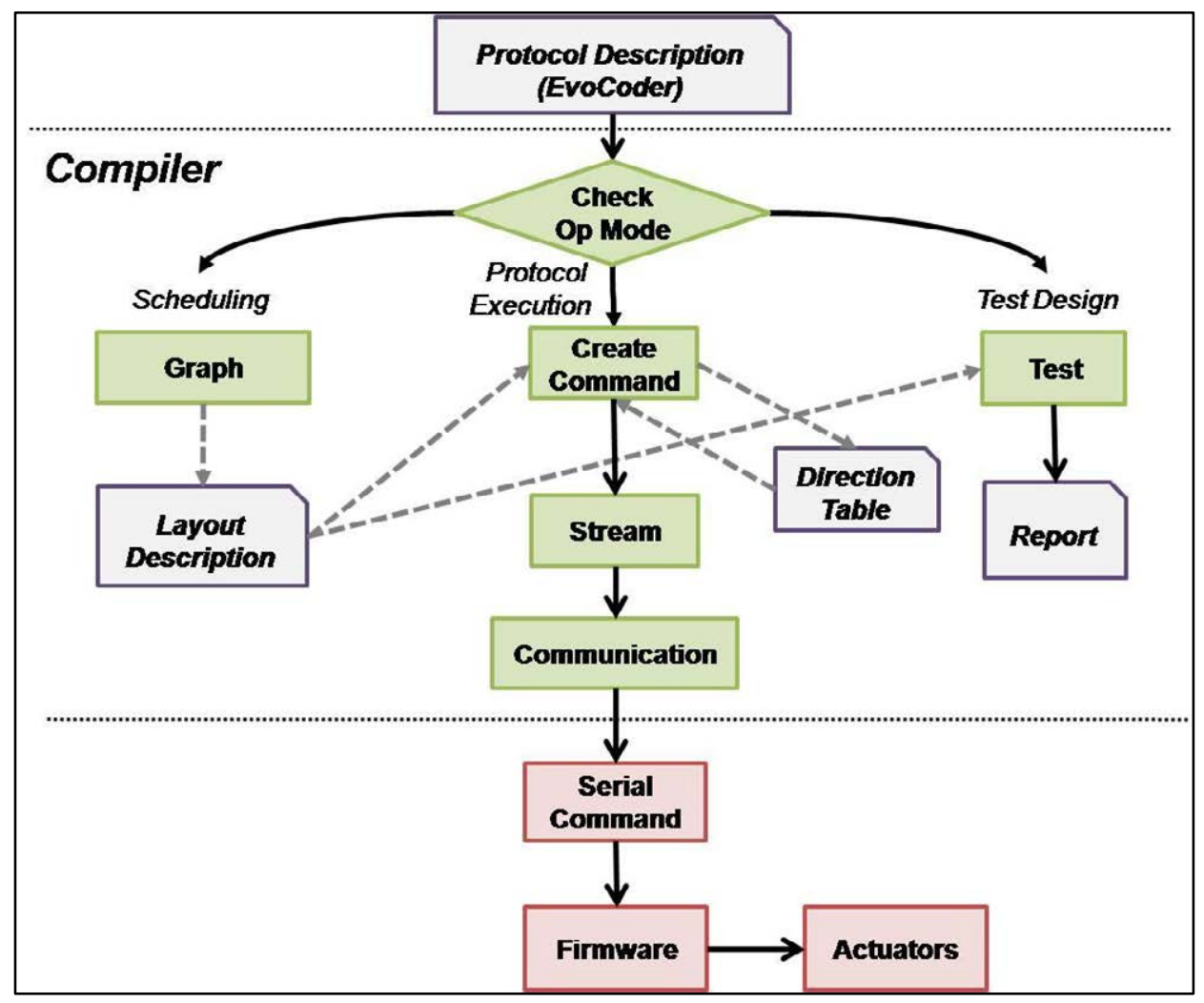

Figure 28 Schematic of the EvoCoder architecture: The three different operation modes Scheduling, Protocol Execution and Test design are depicted in the schematic. The software modules in the compiler are shown in green boxes. The input and output files are shown in white boxes. The software modules on the Arduino side are shown in pink. Unlike the BioBlocks Automation Framework, EvoCoder required a text input of the machine layout. 
The EvoCoder primitives used internally by the Protocol Execution App are -

- Apply Light -

applyLight (const std::string \& sourceld, units::Length wavelength, units::LuminousIntensity intensity) =0;

stopApplyLight (const std::string \& sourceld) = 0;

- Apply Temperature -

applyTemperature (const std::string \& sourceld, units::Temperature temperature) = 0; stopApplyTemperature (const std::string \& sourceld) =0;

- Stir -

stir (const std::string \& idSource, units::Frequency intensity) = 0;

stopStir (const std::string \& idSource) = 0;

- $\quad$ Centrifuge -

centrifugate (const std::string \& idSource, units::Frequency intensity) =0; stopCentrifugate (const std::string \& idSource) = 0;

- $\quad$ Shake -

shake (const std::string \& idSource, units::Frequency intensity) = 0;

stopShake (const std::string \& idSource) = 0;

- Electrophoresis -

startElectrophoresis(const std::string \& idSource, units::ElectricField fieldStrenght) = 0; virtual std::shared_ptr<ElectrophoresisResult> stopElectrophoresis(const std::string \& idSource) = 0; 
virtual units::Volume getVirtualVolume(const std::string \& sourceld) = 0;

- Load Container -

loadContainer(const std::string \& sourceld, units::Volume initialVolume) = 0;

- Meaure OD -

startMeasureOD(const std::string \& sourceld, units::Frequency measurementFrequency, units::Length wavelength) =0;

getMeasureOD(const std::string \& sourceld) = 0;

- Measure Temperature

startMeasureTemperature(const std::string \& sourceld, units::Frequency

measurementFrequency) $=0$;

virtual units::Temperature getMeasureTemperature(const std::string \& sourceld) = 0;

- Measure Luminescence -

startMeasureLuminiscense(const std::string \& sourceld, units::Frequency

measurementFrequency) $=0$;

virtual units::LuminousIntensity getMeasureLuminiscense(const std::string \& sourceld) = 0 ;

- Measure Volume -

startMeasureVolume(const std::string \& sourceld, units::Frequency

measurementFrequency) $=0$;

virtual units::Volume getMeasureVolume(const std::string \& sourceld) = 0; 
- Measure Fluorescence -

virtual void startMeasureFluorescence(const std::string \& sourceld, units::Frequency measurementFrequency, units::Length excitation, units::Length emission) = 0;

virtual units::LuminousIntensity getMeasureFluorescence(const std::string \& sourceld) = 0 ;

- Set a Continuous Flow -

setContinuosFlow(const std::string \& idSource, const std::string \& idTarget, units::Volumetric_Flow rate) $=0$;

stopContinuosFlow(const std::string \& idSource, const std::string \& idTarget) = 0;

- $\quad$ Time Transfer -

virtual units::Time transfer(const std::string \& idSource, const std::string \& idTarget, units::Volume volume) =0;

stopTransfer(const std::string \& idSource, const std::string \& idTarget) =0;

- Mix -

virtual units::Time mix(const std::string \& idSource1, const std::string \& idSource2, const std::string \& idTarget, units::Volume volume1, units::Volume volume2) = 0;

stopMix(const std::string \& idSource1, const std::string \& idSource2, const std::string \& idTarget) = 0;

- $\quad$ Time Step -

setTimeStep(units::Time time) =0;

virtual units::Time timeStep ()$=0$; 


\section{ANNEX III}

The Protocol Specification App in the BioBlocks Automation Framework generates automatically in real-time a protocol workflow. The workflow uses Cytoscape.js libraries to generate the graph. The graph needs data input for defining various node and edge properties to build a graph. The properties used in Cytoscape for generating different types of nodes (containers, conditionals) and edges (discrete and continuous flow) are listed below.

- Node1 - Container Image

- Node1 color - Container Destiny (i.e. if is stored (green) or discarded (red, default))

- Node1 Annotation- Container name

- Node1 shape - Square

- Node1 size - Large

- Node1 annotation/Label - Container name

- Edge1 - Operation Edge

- Edge1 color - Blue

- Edge1 shape - Straight

- $\quad$ Edge1 size - Not being used (default)

- Edge1 type - Solid Line for Discrete operations/ Dotted Line for Continuous flow Operations

- Edge1 annotation/Label - Operation Name \& Time of Operation

- Edge1 length- Not being used

- Node2 - Logic/Conditional

- Node2 color - Orange

- Node2 annotation- Condition

- Node2 size - Small 
- Node2 shape - Diamond

- Edge2 - Condition Edge

- Edge2 color - Not being used

- Edge2 shape - Straight

- $\quad$ Edge2 size - Not being used (default)

- Edge2 length- Not being used (default)

- Edge2 annotation/Label - Condition Type (True/False)

- Box- Loop

- Box color - Light grey

- Box shape- Rectangular

- Box size- custom fit to include operations

- Box annotation- Loop number 


\section{ANNEXIV}

The Machine Specification App in the BioBlocks Automation framework (BAF) automatically generates in real-time the Output code (representation in JSON format). As an example, the Output code of the Turbidostat example is shown below.

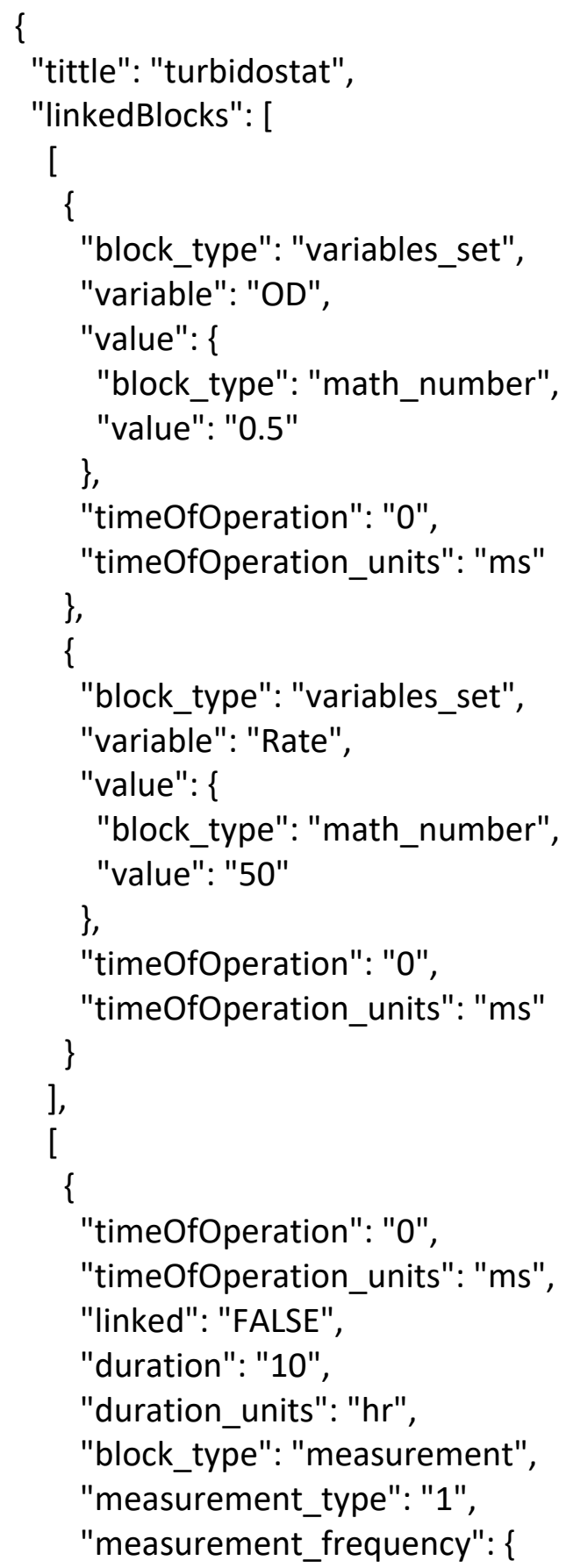




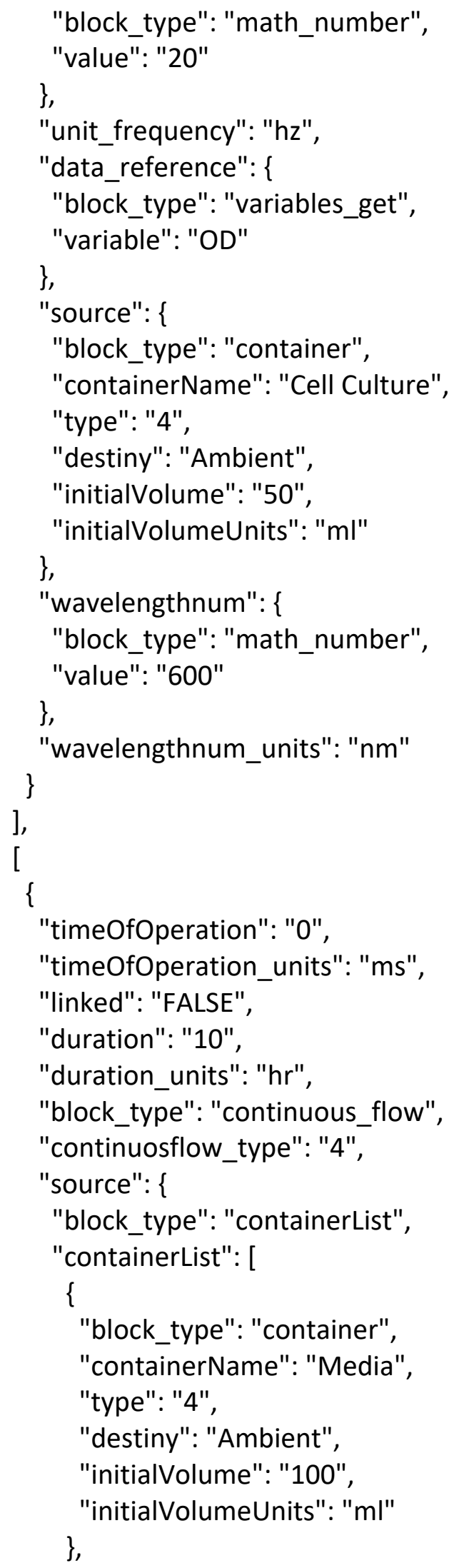




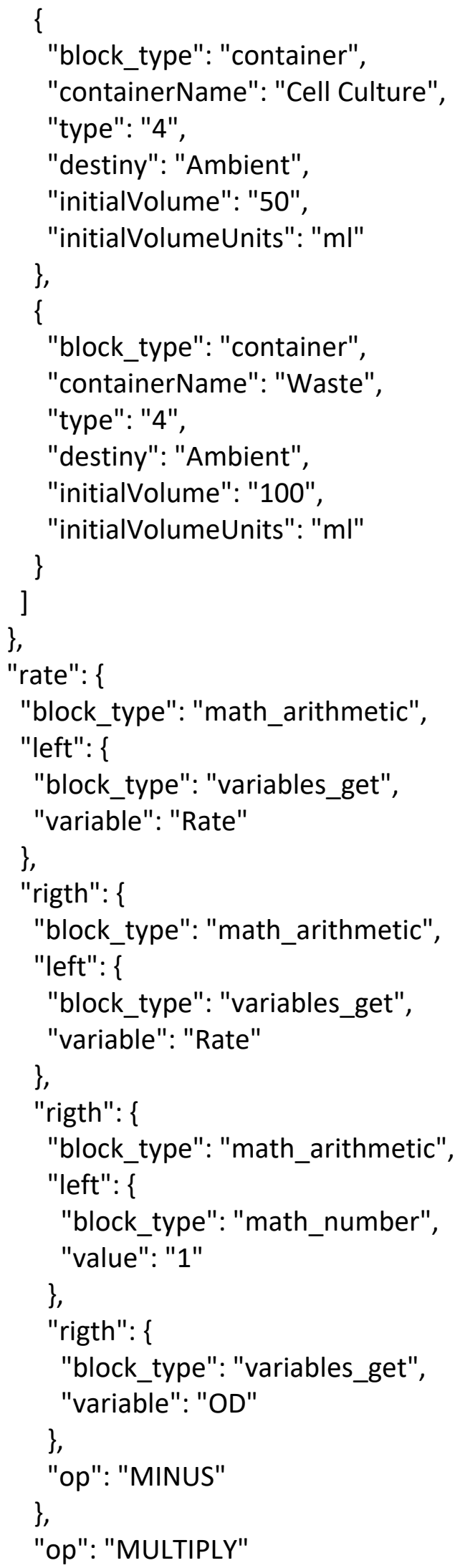




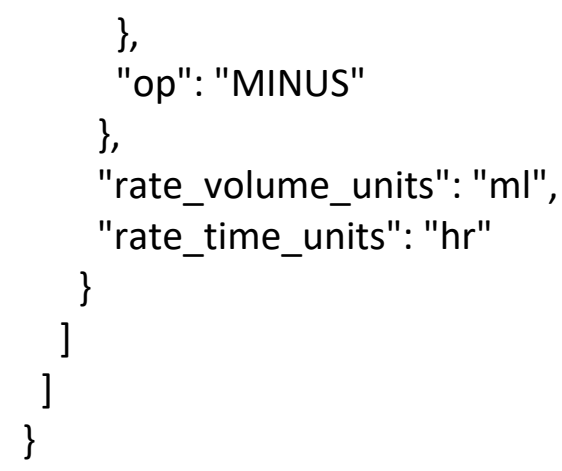

